\title{
CHROMOSOME PAIRING, RECOMBINATION NODULES AND CHIASMA FORMATION IN DIPLOID BOMBYX MALES
}

\author{
by \\ PREBEN BACH HOLM and SØREN WILKEN RASMUSSEN
}

Department of Physiology, Carlsberg Laboratory

Gamle Carlsberg Vej 10, DK-2500 Copenhagen Valby

Keywords: Meiosis, synaptonemal complex, interlocking, chromosome breakage, crossing over, interference, terminalization, centromeres

\footnotetext{
Serial sectioning and three dimensional reconstruction of 82 nuclei divided into 15 well characterized substages covering the period from early zygotene to metaphase $I$ in diploid Bombyx spermatocytes have permitted the following observations and conclusions: 1) At late zygotene, a mean of four chromosome and bivalent interlockings was found per nucleus. 2) During the transition from zygotene to pachytene, all interlockings resolve by breakage and reunion of chromosomes or bivalents. 3) Recombination nodules first appear at early zygotene, reach a maximum of 91 per nucleus by late zygotene, decrease to 55 per nucleus at mid pachytene and increase again to 70-75 towards the end of pachytene. 4) At late zy gotene and pachytene, nodules are essentially distributed at random among bivalents as well as along bivalents. 5) Small, but important deviations from randomness were, however, revealed: Three percent of the bivalents were without nodules at late zygotene, $8 \%$ at early pachytene but at mid and late pachytene, only $2 \%$ were devoid of nodules. 6) From mid pachytene, an increasing fraction of the recombination nodules becomes larger and more irregular in shape. These are termed chromatin nodules and at the pachytene-diplotene transition, nearly all nodules are of this type. 7) During early diplotene, the bulk of the chromatin decondenses leaving only about 60 major condensed domains. These domains originate from chromatin nodules and are frequently associated with a piece of synaptonemal complex which in its central region contains a dense core resembling a recombination nodule. 8) At late diplotene, the condensed domains of the bivalents consist of two dense chromatin segments, one on each of the homologues, and bridged by a complex circular structure giving the regions a characteristic tripartite appearance. 9) At mid diakinesis, the circular structures are located in chromatin bridges - chiasmata - between the homologous chromosomes. 10) The number of chiasmata per nucleus is constant from mid diplotene to mid diakinesis and is distributed among the bivalents similar to the distribution of recombination nodules at mid and late pachytene. 11) The circular chiasma components are eliminated from late diakinesis until metaphase I. 12) Finally, each metaphase I bivalent contains four distinct localized centromeres, two by two facing opposite spindle poles.
} 


\section{INTRODUCTION}

Meiosis in eukaryotes has attracted and fascinated scientists for nearly a century. Throughout this period geneticists and cytologists have in a concerted interplay, though not always in absolute harmony, contributed to the interpretation of the meiotic process, eventually resulting in the establishment of a basic framework for meiosis, a framework which has essentially remained unaltered for the last 50 years. Although numerous modifications of meiosis have been described in different species and different taxonomic groups of fungi, plants and animals, the fundamental events of meiosis are highly conserved (see for example DarLINGTON, 16).

In recent years, analysis is focusing on the genetic regulation of the meiotic events $(2,26$, $87)$ and on the biochemical aspects. HotTA and STERN $(78,77)$ thus have demonstrated that the normal progression of meiosis is accompanied by the synthesis of specific proteins and enzymes of importance for pairing and recombination as well as by specific events in DNA metabolism at premeiotic interphase, zygotene and pachytene. The discovery of the synaptonemal complex has provided an incitement to continue the classical cytological approach, now at the submicroscopic level. Such studies have been performed either on single sections, by three dimensional reconstructions from serial sections or more recently by spreading of whole nuclei. The technique of serial sectioning and three dimensional reconstruction is at present the only method available which allows a quantitative investigation of chromosome morphology and behaviour in intact nuclei. Although being the most laborious, this technique combines an optimal preservation of nuclear fine structure with the possibility of determining the exact positions of chromosomes and bivalents at most meiotic stages.

The available evidence on chromosome pairing and synaptonemal complex formation (see $24,57,69,84$ for reviews) can briefly be summarized as follows: Homologous chromosomes appear to be fortuitously distributed within the premeiotic interphase nucleus. At leptotene, each chromosome organizes a lateral component, the telomeres become attached to the nuclear envelope and after the congression of the telomeres whereby a chromosome bouquet is formed, chromosome pairing and synaptonemal formation start. In some organisms synaptonemal complex formation is initiated preferentially from the telomeres $(63,66)$ while in others, complex formation may also occur interstitially $(23,28,86)$. In both cases, this pairing occurs between homologous chromosomes or chromosome regions (69). Chromosome and bivalent interlocking are regular features of the zygotene pairing and are corrected by breakage and reunion of the involved chromosomes $(66,69)$. Instabilities of the zygotene pairing may, in the absence of crossing over, be corrected by partial dissolution of the central region of the synaptonemal complex followed by a second round of synaptonemal complex formation which can also involve nonhomologous chromosomes and chromosome segments $(30,65,68,69)$.

At the electron microscope level, quantitative information on crossing over and particularly on chiasma formation and disjunction is more scarce. In 1975, CARPENTER (11) reported that in Drosophila, there is good correaltion between the number and distribution of electron dense spherical nodes - recombination nodules - and the number and distribution of crossovers, and suggested that recombination nodules were somehow associated with crossing over. This hypothesis has since then been supported by a number of investigations $(9,12,13,25,66,86)$. Later, the hypothesis was extended by the proposal that also nonreciprocal exchanges are mediated by recombination nodules $(12,66,69)$. The hypothesis has received further support by the observation that recombination deficient organisms such as female Bombyx lack recombination nodules at mid pachytene (69), whereas meiotic mutants in Drosophila, partially deficient in recombination, at least in some cases show a proportionate reduction in the number of recombination nodules (13).

Most of the evidence presented above is still at the level of correlations and the chemical composition of the recombination nodules is entirely unknown. Considering the present evidence it is, however, apparent that recombination nodules are prerequisites for recombination rather than the result of the recombination events as suggested by Holliday (27).

Very little is at present known about the mechanisms regulating the distribution of re- 
combination nodules or exchanges among and along the bivalents. From observations in Drosophila, CARPENTER (13) favoured the idea that the information for the placement of the nodule is embodied in the nodule itself. Evidence from human spermatocytes on the other hand suggest that with few exceptions, nodules are basically distributed at random along and among bivalents at late zygotene and early pachytene $(31,66)$.

The chiasmatype theory originally formulated by JANSSENS (35) states that chiasmata originate from crossovers, a proposal which is now widely accepted and supported by a large body of evidence (see for example WHITEHOUSE, 85). Theoretically, it should therefore be possible to detect a morphological continuity between bivalent regions containing recombination nodules and chiasmata. In Sordaria, ZICKLER (86) provided the first evidence for the relationship between recombination nodules and chiasmata by demonstrating that at diplotene recombination nodules were present in retained pieces of synaptonemal complex. This observation is consistent with the proposal by WestergaARD and VON WETTSTEIN $(82,83)$ that the early diplotene chiasma is mechanically stabilized by a retained fragment of the synaptonemal complex. This so-called "synaptonemal complex chiasma" was reported to be converted into a stable wchromatin chiasma « at later stages $(73,83)$.

The present analysis, being based on a continuous sequence of reconstructed nuclei covering the period from early zygotene to metaphase I, provides direct evidence of a morphological continuity between bivalent regions containing recombination nodules and chiasmata. Furthermore, it demonstrates that chiasmata are characteristic and elaborate structures in accordance with their role in holding together the homologues during diplotene and diakinesis. The present study also describes in detail the chromosome pairing and synaptonemal complex formation in Bombyx spermatocytes, and demonstrates extensive interlocking at late zygotene and its subsequent resolution by breakage and reunion of chromosomes and bivalents. A preliminary report including some of these results has been presented previously (31).

\section{MATERIALS AND METHODS}

The materials used in the present study were kindly provided by the late Professor B. L. Astaurov, Institute of Developmental Biology, Academy of Sciences USSR, Moscow and by Dr. H. AKal, Sericultural Experiment Station, Tsukuba, Ibaraki, 305 Japan. Nuclei with the numbers $1-28,37-40,46-50,62-64$ and $78-$ 81 are from the Russian material while the nuclei with the numbers $29-36,41-45,51-61$, 61a and 65-77 are from the Japanese. Differences were not observed between the Russian and the Japanese materials.

The procedure for removing the reproductive organs of Bombyx and their subsequent fixation, dehydration and embedding has been described previously (63). Thick sections $(2-4 \mu \mathrm{m})$ were cut and examined in the light microscope in order to identify cell cysts in the desired stage of development. Series of thin sections were cut, photographed at a primary magnification of 48000 times and reconstructed as described by Holm and Rasmussen (29). The projected lengths were measured and the real lengths calculated with the aid of a Hewlett Packard digitizer (HP9864A) and calculator as described by RASMUSSEN and Holm (66). An idiogram of the chromosome complement of each nucleus was drawn with a HP9872 four colour plotter. The theoretical distributions of nodule-nodule and noduletelomere distances were constructed with the aid of a Honeywell Bull $\mathrm{H} 6000$ computer as described in section 3.19.3.

\section{RESULTS}

\subsection{General aspects of spermatogenesis}

The life cycle as well as the histology of the reproductive organs of the domesticated silkworm, Bombyx mori, are well characterized (see TAZIMA, 79 and TAZIMA et al., 80 for reviews): In the larvae, each of the two testes consists of four conical follicles. The primary spermatogonia located near the blind end of each follicle divide giving rise to secondary spermatogonia, which by synchronous divisions in the second instar form cysts of primary spermatocytes. Each lobe eventually constains about 1500 cysts consisting of 64 spermatocytes enclosed by a monolayer of follicle cells. All the spermatocytes of a cyst are joined by ring canals (Figures 21 and 25) and are 


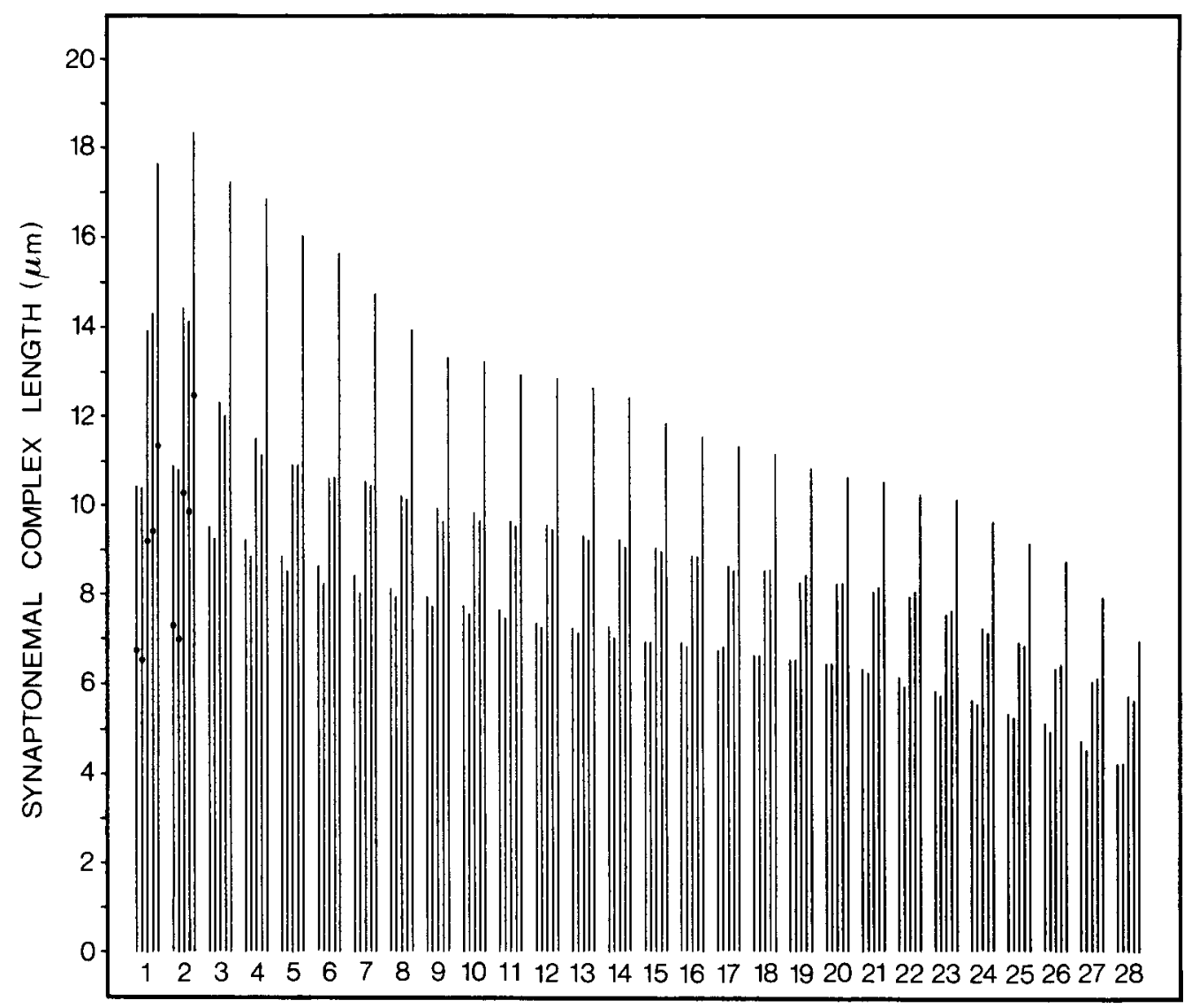

\section{CHROMOSOME NUMBER}

Figure 1. Idiogram showing the mean absolute synaptonemal complex length of each of the 28 bivalents at late zygotene (leftmost lines), early pachytene (second lines), mid pachytene (middle lines), late pachytene (fourth lines) and pachytene-diplotene (rightmost lines). The position of the knob on bivalent 1 and the nucleolus organizer region on bivalent 2 are denoted by circles on the respective bivalents.

in the same stage of development when entering the meiotic prophase (39). At the beginning of the fourth instar, most spermatocytes are in zygotene or pachytene and contain distinct synaptonemal complexes $(40,41)$. Diakinesis, metaphase I and later stages appear in the fifth instar. The whole meiotic prophase has been estimated to last 10-11 days (71).

\subsection{Classification of bivalents}

Identification and classification of the 28 Bombyx bivalents are met with difficulties, mainly because of the absence of centromeric heterochromatin and the scarcity of morphological markers, but also because of the relatively small differences in length between the longest and the shortest chromosomes (Figure 1). Only chromosomes 1 and 2 can be identified with certainty (63), chromosome 1 by a knob dividing the chromosome into arms with relative lengths of 1 and 1.8 (mean of all stages) and chromosome 2 by the nucleolus organizer region which divides the chromosome into arms of relative lengths 1 and 2.1. For the remaining 52 chromosomes, classification is restricted to a simple ranking according to lateral component length (Figure 1). Obviously, this restrains the 


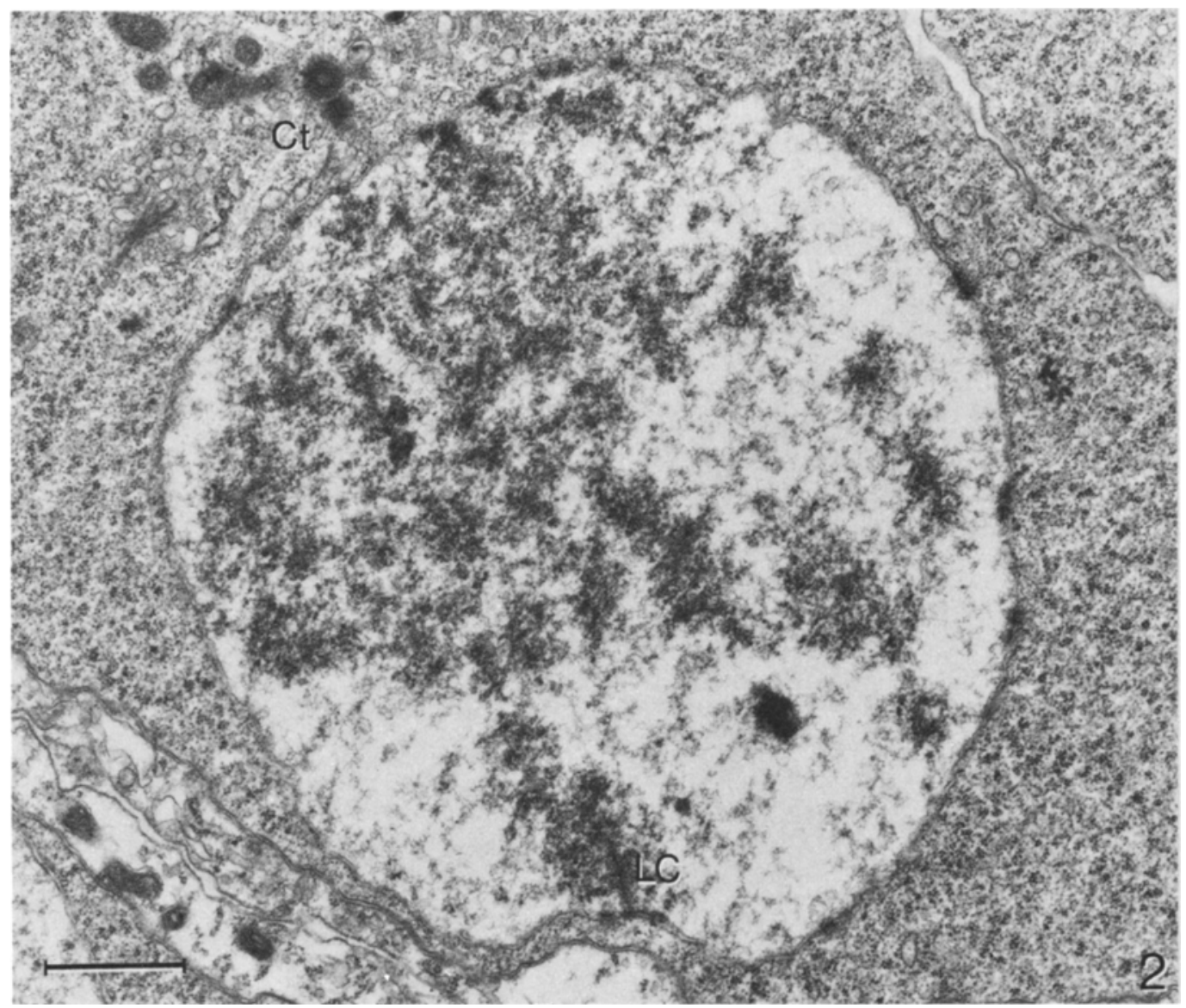

Figure 2. Survey micrograph of an early zygotene nucleus. $\mathrm{Ct}$, centrioles; LC, lateral component. $(\mathrm{Bar}=1 \mu \mathrm{m})$.

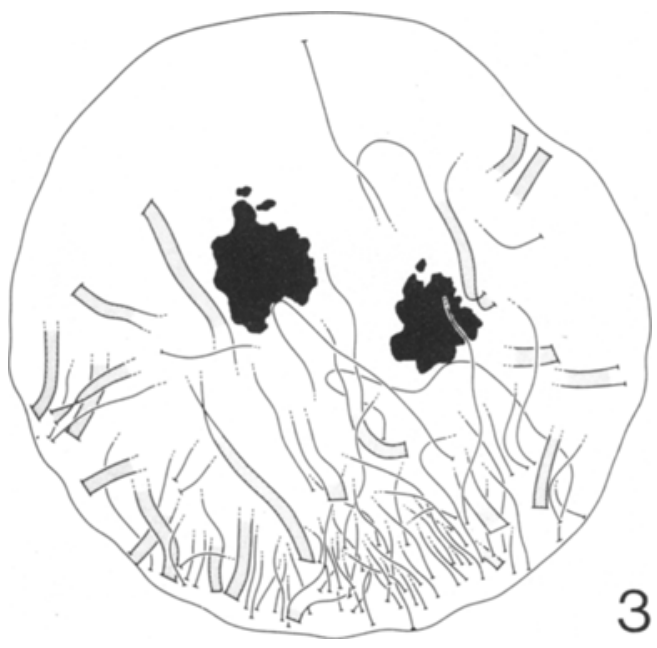

reliability of the classification and it is to be expected that the number assigned to a certain bivalent will be different in different nuclei. Bivalents other than numbers 1 and 2 were occasionally found to carry morphological markers in the form of various chromatin condensations especially at mid and late pachytene. Although of potential use, their presence was too

Figure 3. Reconstruction of an early zygotene nucleus (number 11). All 112 telomeres are attached to the nuclear envelope and despite yet incomplete lateral component organization, synaptonemal complex formation is initiated at several telomere regions. (Magnification approximately 9.000 times). 
erratic to be of any value in the classification of Bombyx chromosomes at the electron microscope level.

\subsection{Early zygotene}

The zygotene stage of meiosis is at the ultrastructural level characterized by the initiation of chromosome pairing and synaptonemal complex formation and by a pronounced cellular polarity. Four nuclei (numbers $11,12,38,40$ ) were serially sectioned and partially reconstructed (Figures 2 and 3 ). In three of the nuclei (11, 12 and 40), pairing and synaptonemal complex formation of the 28 pairs of homologues were in progress: 112,112 and 111 of the 112 telomeres were attached to the inner membrane of the nuclear envelope and all attachment sites had congregated giving rise to a chromosome bouquet. In nucleus 38 , the chromosome bouquet was less pronounced, only 108 telomeres were attached to the nuclear envelope and most of the chromosomes were still unpaired.

As shown in Figure 3, the lateral components are not yet continuous between the telomere regions and synaptonemal complex formation is at this stage confined to terminal or subterminal parts of the homologues. In three of the four nuclei, the nucleolus organizer regions were located in different parts of the nuclei while in the last nucleus (number 38) the two nucleoli had fused and synaptonemal complex formation was complete between the organizer chromosomes.

In the cytoplasm, most of the mitochondria and Golgi apparatuses were concentrated outside the bouquet region, partly surrounding the two pairs of centrioles (Figure 2). A few microtubules radiate out from the centrioles into the cytoplasm while others appear to be associated with the nuclear envelope.

\subsection{Mid zygotene}

One mid zygotene nucleus was serially sectioned and completely reconstructed (number 39 ). As shown in the reconstruction (Figure 4), the lateral components are now nearly continuous, and pairing and synaptonemal complex formation are in progress in several pairs of homologues. A substantial asynchrony in the degree of synaptonemal complex formation is evident:
Three pairs of homologues have completed synaptonemal complex formation, complex formation is in progress in 17 pairs of homologues, while 16 chromosomes, including the nucleolus organizing chromosomes, are still entirely unpaired. In most cases, pairing and synaptonemal complex formation are initiated at the telomere regions. Possible interlockings were noted as well as a few discontinuities of the lateral components. The latter may signify either incomplete lateral components or chromosome breakage associated with resolving interlocks (cf. section 3.5.3.).

At this stage, as well as in early zygotene, dense nodes - recombination nodules - are readily identified associated with the central region of the synaptonemal complex (Figure 4).

\subsection{Late zygotene}

\subsubsection{General aspects}

The late zygotene stage was analyzed by reconstruction of eight nuclei from two individuals. Nuclei numbers 21, 22 and 24 are from the first individual and nuclei numbers 37,46 , 47,49 and 50 from the second.

The fine structure of a late zygotene nucleus is shown in Figure 5 and a complete reconstruction in Figure 6. The nuclear and cytoplasmic polarity is pronounced as in early and mid zygotene, and in all nuclei the 112 telomeres

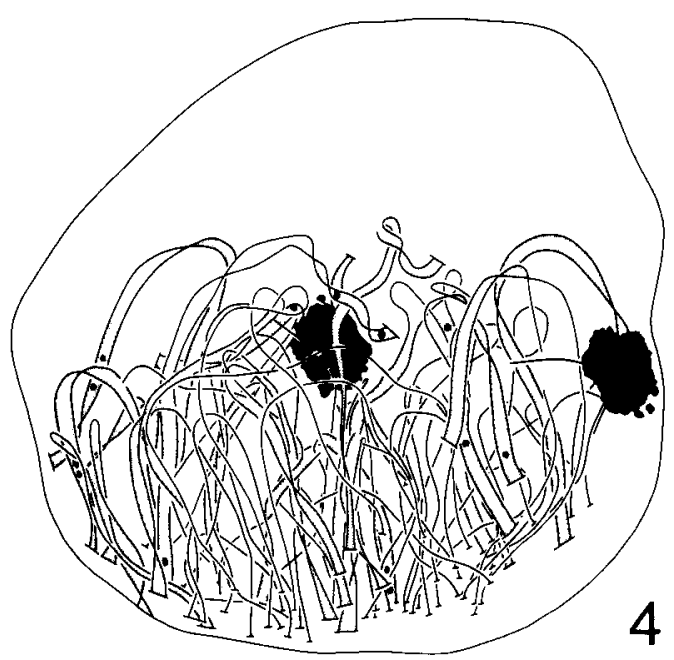

Figure 4. Reconstruction of a mid zygotene nucleus (number 39). 


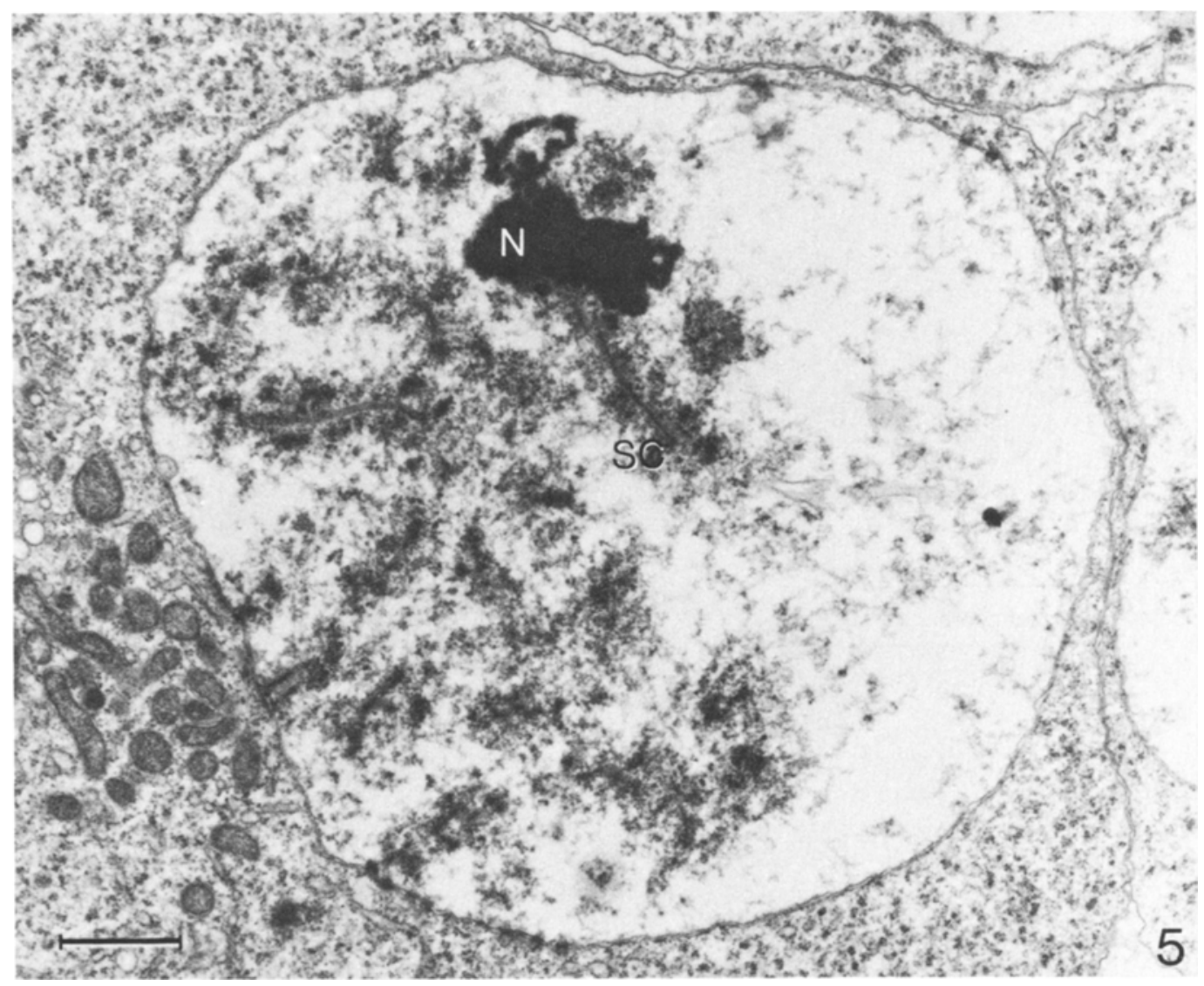

Figure 5. Survey micrograph of a late zygotene nucleus. SC, synaptonemal complex; N, nucleolus. $(\mathrm{Bar}=1 \mu \mathrm{m})$.

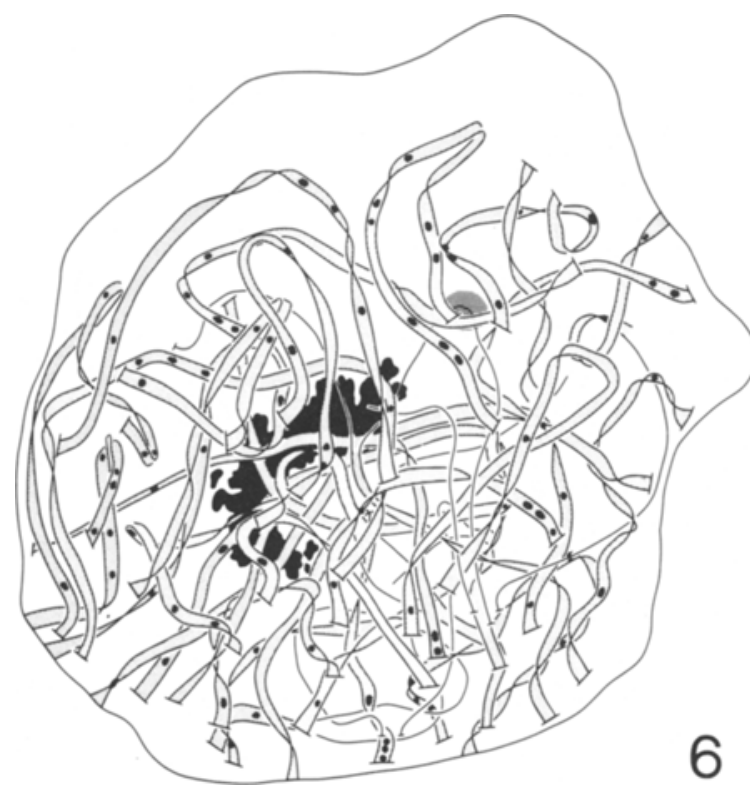

were attached to the nuclear membrane. The lateral components have increaced in density and are continuous between the telomeres of each chromosome. Synaptonemal complex formation is completed in most regions of the homologues and many recombination nodules are present in the central region of the complex as shown in Figure 6 (see also section 3.10.).

The total length of the chromosome complement is given in Table I. The length of the partially paired bivalents is calculated by adding the synaptonemal complex length of the paired region to the mean lateral component length of

Figure 6. Reconstruction of a late zygotene nucleus (number 47). The partially paired bivalents of this nucleus are shown in Figure 17. (Magnification approximately 9,000 times). 
Table I

Total synaptonemal complex (SC) length and number of recombination nodules (RN) at late zygotene in Bombyx spermatocytes.

\begin{tabular}{cccccccc}
\hline $\begin{array}{c}\text { Nucleus } \\
\text { number }\end{array}$ & $\begin{array}{c}\text { Complement } \\
\text { lengtha) } \\
(\mu \mathrm{m})\end{array}$ & $\begin{array}{c}\text { SC length } \\
(\mu \mathrm{m})\end{array}$ & $\begin{array}{c}\text { Percent } \\
\text { pairing }\end{array}$ & $\begin{array}{c}\text { Number } \\
\text { of RN }\end{array}$ & $\begin{array}{c}\text { Expected } \\
\text { number } \\
\text { of RN }\end{array}$ & $\begin{array}{c}\text { SC length } \\
\text { per RN } \\
(\mu \mathrm{m})\end{array}$ & $\begin{array}{c}\text { Number of } \\
\text { bivalents } \\
\text { without RN's }\end{array}$ \\
\hline 21 & 187 & 164 & 88 & 72 & 82 & 2.3 & $1 \mathrm{~b})$ \\
22 & 189 & 185 & 98 & 80 & 82 & 2.3 & $1 \mathrm{~b})$ \\
24 & 177 & 162 & 91 & 75 & 82 & 2.2 & $2 \mathrm{c})$ \\
37 & 221 & 159 & 75 & 106 & 141 & 1.5 & 1 b) \\
46 & 211 & 198 & 94 & 100 & 106 & 2.0 & 0 \\
47 & 222 & 201 & 91 & 100 & 110 & 2.0 & 0 \\
49 & 198 & 179 & 90 & 90 & 100 & 2.0 & 1 b) \\
50 & 222 & 192 & 86 & 106 & 123 & 1.8 & 1 b) \\
\hline Mean \pm s.d. & $202 \pm 17$ & $180 \pm 17$ & 89 & $91 \pm 14$ & $103 \pm 21$ & 2.0 & 0.9 \\
\hline
\end{tabular}

a) Total lateral component length divided by two.

b) Incompletely paired bivalent.

c) One completely and one incompletely paired bivalent.

the unpaired region. Generally, the two unpaired lateral components were of equal length. The length of the complement ranges from 177 to $222 \mu \mathrm{m}$, with a mean value of $202 \pm 17 \mu \mathrm{m}$.

\subsubsection{Chromosome pairing}

The general pattern of chromosome pairing observed at early and mid zygotene is confirmed by the analysis of the eight late zygotene nuclei. In only one case had pairing and synaptonemal complex formation occurred between apparently nonhomologous chromosome regions. A short interstitial synaptonemal complex segment had formed between chromosomes 1 and 9 , probably due to an interlocking of chromosome 1 preventing a regular pairing of this bivalent. In the rest of the bivalents, pairing and synaptonemal complex formation appeared to be strictly homologous.

All nuclei contained chromosome and bivalent interlockings (see section 3.5.3.) and most of the cases of incomplete bivalent formation were due to interlockings or their resolution. The remaining cases where pairing was not impeded by interlockings can be summarized as follows: 1) Completely unpaired chromosomes were found in only two cases (chromosomes 7 and 17). 2) In 6 cases, pairing and synaptonemal complex formation had started from one end only, leaving the other end unpaired (bivalents 1, 6, 8, 16, 22 and 26). 3) In 9 cases (bivalents 1, 1, 3, 3, 5, 12, 19,26 and 28) pairing and synaptonemal complex formation had startet from both ends with the middle region still unpaired.

Interstitial initiation of pairing and synaptonemal complex formation were noted in 8 bivalents (numbers 1, 1, 2, 4, 5, 5, 17 and 21) and with one exception (bivalent 5), all cases were found among bivalents involved in chromosome or bivalent interlockings. It is thus apparent that telomeric initiation of synapsis is the rule in Bombyx spermatocytes but that in cases where movements of the homologues are impeded by interlockings, bivalents may initiate synaptonemal complex formation interstitially.

With the exception of bivalent 1 , which on the average is paired over $53 \%$ of its length, all the bivalents exhibit approximately the same degree of pairing as shown in Table II. The low degree of pairing in bivalent 1 can accordingly not be attributed to its length alone. More likely, the knob on this bivalent impedes synaptonemal complex formation in accordance with the situation reported in tetraploid Bombyx oocytes (68). 
Table II

Mean lateral component ( $L C$ ) length, number of recombination nodules (RN), interlockings and breaks of the 28 bivalents of 8 Bombyx spermatocytes at late zygotene.

\begin{tabular}{|c|c|c|c|c|c|c|}
\hline \multirow{2}{*}{$\begin{array}{l}\text { Bivalent } \\
\text { number }\end{array}$} & \multirow{2}{*}{$\begin{array}{l}\text { LC lengtha) } \\
(\mu \mathrm{m} \pm \text { s.d. })\end{array}$} & \multirow{2}{*}{$\begin{array}{l}\text { Number of } \\
\operatorname{RN}( \pm \text { s.d. })\end{array}$} & \multirow{2}{*}{$\begin{array}{l}\text { Percent } \\
\text { paired }\end{array}$} & \multirow{2}{*}{$\begin{array}{l}\text { Number of times } \\
\text { interlocking } \\
\text { other chromosomes } \\
\text { or bivalents }\end{array}$} & \multicolumn{2}{|c|}{ Number of breaks } \\
\hline & & & & & Chromosome & Bivalent \\
\hline 1 & $10.4 \pm 0.9$ & $2.5 \pm 1.5$ & 53 & 3 & 0 & 1 \\
\hline 2 & $10.9 \pm 0.8$ & $5.0 \pm 1.6$ & 93 & 0 & 0 & 1 \\
\hline 3 & $9.5 \pm 0.8$ & $2.3 \pm 1.5$ & 92 & 0 & 1 & 0 \\
\hline 4 & $9.2 \pm 0.8$ & $3.0 \pm 1.3$ & 81 & 5 & 1 & 2 \\
\hline 5 & $8.8 \pm 0.7$ & $4.0 \pm 2.2$ & 84 & 4 & 0 & 0 \\
\hline 6 & $8.6 \pm 0.8$ & $3.9 \pm 1.4$ & 85 & 0 & 2 & 0 \\
\hline 7 & $8.4 \pm 0.9$ & $3.6 \pm 2.3$ & 87 & 0 & 0 & 0 \\
\hline 8 & $8.1 \pm 0.8$ & $3.3 \pm 2.3$ & 96 & 1 & 1 & 1 \\
\hline 9 & $7.9 \pm 0.8$ & $4.5 \pm 2.5$ & 98 & 1 & 0 & $i$ \\
\hline 10 & $7.7 \pm 0.8$ & $3.9 \pm 2.2$ & 95 & 0 & 0 & 0 \\
\hline 11 & $7.6 \pm 0.7$ & $3.6 \pm 1.8$ & 93 & 1 & 2 & 0 \\
\hline 12 & $7.3 \pm 0.6$ & $3.0 \pm 2.4$ & 95 & 1 & 0 & 1 \\
\hline 13 & $7.2 \pm 0.6$ & $3.8 \pm 1.7$ & 100 & 0 & 0 & 0 \\
\hline 14 & $7.2 \pm 0.7$ & $3.4 \pm 1.1$ & 87 & 4 & 0 & 0 \\
\hline 15 & $6.9 \pm 0.5$ & $2.3 \pm 1.0$ & 87 & 2 & 0 & 0 \\
\hline 16 & $6.9 \pm 0.6$ & $3.5 \pm 1.7$ & 95 & 0 & 0 & 0 \\
\hline 17 & $6.7 \pm 0.6$ & $2.4 \pm 2.3$ & 74 & 4 & 0 & 0 \\
\hline 18 & $6.6 \pm 0.6$ & $3.6 \pm 1.5$ & 100 & 0 & 0 & 0 \\
\hline 19 & $6.5 \pm 0.6$ & $3.9 \pm 2.9$ & 86 & 1 & 0 & 0 \\
\hline 20 & $6.4 \pm 0.6$ & $3.0 \pm 1.2$ & 90 & 4 & 0 & 0 \\
\hline 21 & $6.3 \pm 0.6$ & $3.3 \pm 1.8$ & 85 & 0 & 1 & 0 \\
\hline 22 & $6.1 \pm 0.6$ & $3.4 \pm 2.3$ & 91 & 1 & 2 & 1 \\
\hline 23 & $5.8 \pm 0.7$ & $2.8 \pm 1.3$ & 93 & 2 & 1 & 0 \\
\hline 24 & $5.6 \pm 0.6$ & $2.8 \pm 1.9$ & 99 & 2 & 2 & 0 \\
\hline 25 & $5.3 \pm 0.6$ & $2.8 \pm 1.8$ & 93 & 0 & 0 & 0 \\
\hline 26 & $5.1 \pm 0.4$ & $2.4 \pm 1.5$ & 87 & 0 & 0 & 0 \\
\hline 27 & $4.7 \pm 0.4$ & $2.6 \pm 1.8$ & 98 & 1 & 0 & 1 \\
\hline 28 & $4.2 \pm 0.8$ & $2.9 \pm 2.0$ & 93 & 0 & 0 & 0 \\
\hline
\end{tabular}

a) Mean lateral component length of the two homologues.

\subsubsection{Interlockings and breaks}

Interlocking is a regular feature of zygotene chromosome pairing as judged by its high frequency in the 8 late zygotene nuclei analyzed (Tables II, III and IV). Chromosome interlockings, i.e., the entrapment of one lateral component of a partially paired bivalent between the homologues of another partially paired bivalent (Figure 7), were present with a mean frequency of 1.6. per nucleus. Bivalent interlockings (Figure 8), i.e., the entrapment of both homo- logues between the unpaired lateral components of an unpaired interstitial segment in another bivalent, had occurred with a mean frequency of 2.4 per nucleus. In a few cases, more than one chromosome or bivalent were entrapped within the same unpaired bivalent region. The reconstruction shown in Figure 9 and the series of micrographs with the accompanying reconstruction in Figures 10 and 11 are examples of such double interlockings.

The total number of cases in all 8 nuclei where 
a partially paired bivalent has interlocked other chromosomes or bivalents are listed in Table II. These data demonstrate that there is a low correlation, if any at all, between the length of a bivalent and the frequency with which it interlocks other chromosomes and bivalents.

Also chromosome and bivalent breaks, as recognized by major discontinuities of lateral components and synaptonemal complexes, are frequent at late zygotene (Tables II, III and IV). The mean number of chromosome and bivalent breaks in the eight late zygotene nuclei amounts to 1.6 and 1.1 respectively, which adds up to a total number of 3.8 chromosome breaks per nucleus. A substantial variation in the number of breaks per nucleus is observed, ranging from complete absence of breaks in nucleus 22 to the large number of breaks in nuclei 49 and 24 (Table IV). In the latter nucleus, five pairs of homologues located in the middle of the bouquet region were all broken at several sites and the precise number of breaks could not be determined with certainty. The absence of breaks in nucleus 22 may be attributed to its more advanced state of pairing (Table I, 98\%) since virtually all breaks disappear by reunion and repair of the broken ends before early pachytene (see section 3.6.2.).

Most, if not all, of the breaks appear to originate as intermediates in the resolution of interlockings: of the 22 chromosome and bivalent breaks listed in Tables III and IV, six of the chromosome breaks and two of the bivalent breaks had clearly taken place within previously interlocked regions. An example is given in a series of micrographs in Figure 12 and in the reconstructions in Figure 13. In the rest of the cases, the broken chromosome and bivalent ends were free in the nucleoplasm, often separated by distances up to several $\mu \mathrm{m}$ (Figures 14 and 15).

The number of breaks of the different chromosomes and bivalents are listed in Table II. As in the case for interlockings, there is no clear correlation between the length of a particular chromosome and the frequency of breakage.

Half of the nuclei (numbers 24, 37, 47, 50) contained very complex chromosome configurations involving up to eight bivalents with several interlockings and breaks (Figures 16 and 17), and it is conceivable that also the other four nuclei, which were in a more advanced state of pairing, have passed through a similar stage of massive interlocking and chromosome breakage. In addition, resolution of interlockings by breakage and reunion of chromosomes or bivalents may occur also during earlier stages and the observed number of 7.9 interlockings and chromosome breaks per nucleus may therefore represent only a minimum number.

\subsection{Early pachytene}

\subsubsection{General aspects}

The results presented below are based on reconstructions of 16 nuclei from 5 individuals (nuclei 5-10, 13-16, 23, 25-28 and 48). As in mid and late zygotene, both pairs of centrioles are located close together outside the bouquet region surrounded by cytoplasmic organelles (Figure 19). The early pachytene nucleus is characterized by complete pairing of all bivalents (Figures 18 and 20), the only exception being two nuclei (numbers 14 and 25) in which pairing of chromosome 1 was not yet completed due to interlockings (see section 3.6.2.). The bouquet configuration is still present and the telomeres are without exception attached to the inner membrane of the nuclear envelope. The lateral components and the central region of the synaptonemal complex are at this stage more distinct than at late zygotene, and recombination nodules are readily identified associated with the central region of the complex (cf. section 3.10.).

The total complement length per nucleus is given in Table V. The length varies from 182 to $214 \mu \mathrm{m}$ with a mean value of $198 \pm 10 \mu \mathrm{m}$.

\subsubsection{Interlockings and breaks}

The vast majority of the interlockings and breaks seen at late zygotene has disappeared by early pachytene. Only two interlockings were found in the 16 completely reconstructed early pachytene nuclei and both were bivalent interlockings (chromosomes 1 interlocking bivalent 2 in nucleus 14 and bivalent 5 in nucleus 25). In addition, three cases of bivalent breakage were observed (bivalent 18 in nucleus 7; bivalent 28 in nucleus 8; bivalent 3 in nucleus 48). Altogether, the mean number of aberrations per nucleus is 0.5 . When compared to the 7.9 aberrations per nucleus found at late zygotene, it is evident that 


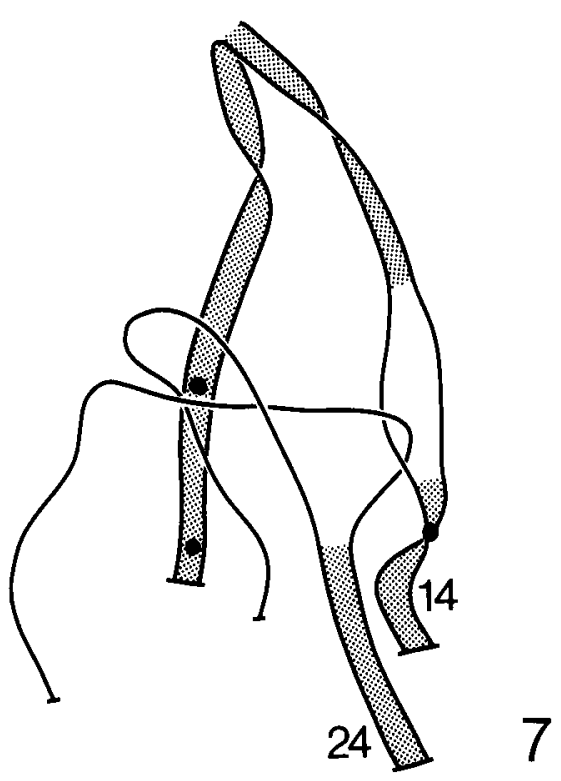

Figure 7. Chromosome interlocking at late zygotene (nucleus 49). One homologue of bivalent 24 is interlocked by the lateral components of the unpaired segment in bivalent 14 .

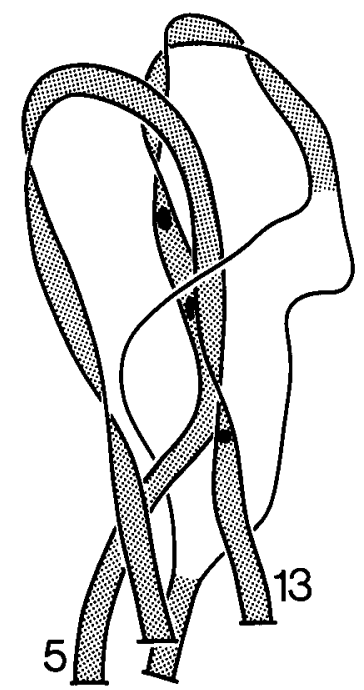

Figure 8. Bivalent interlocking at late zygotene (nucleus 21 ). The completely paired bivalent 13 is interlocked by the unpaired segment of bivalent 5 . Also the long paired segment of bivalent 5 passes through the unpaired region resulting in self interlocking of bivalent 5 .

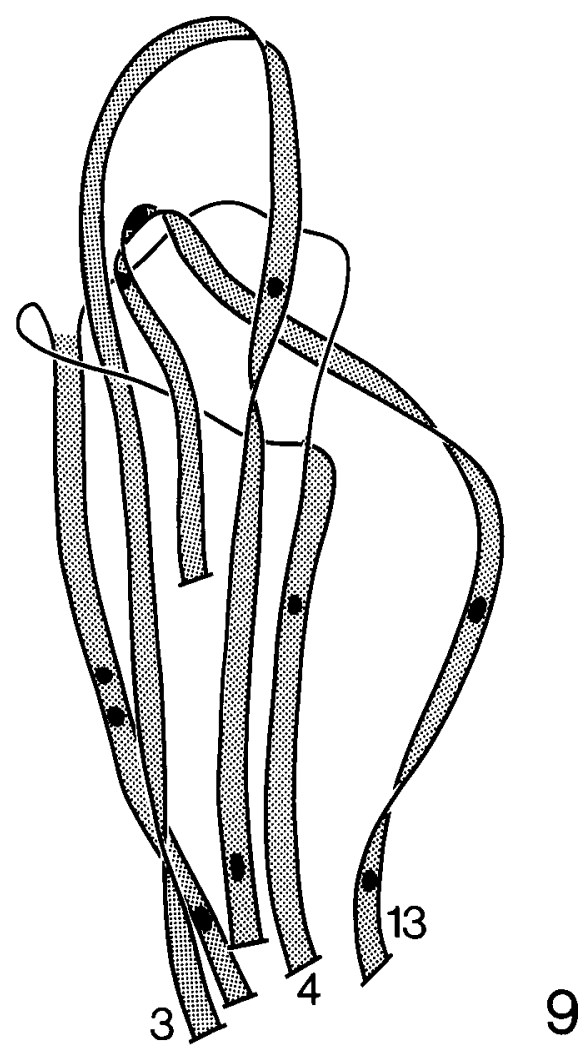

Figure 9. Double bivalent interlocking at late zygotene (nucleus 22). Bivalents 3 and 13 are both interlocked by the unpaired segment of bivalent 4 .

the resolution of interlockings by chromosome or bivalent breakage is followed by a reunion and repair of the broken ends. Furthermore, the repair process appears to be highly specific as judged by the absence of chromosome rearrangements in the analyzed pachytene nuclei. Reunion of broken bivalent ends appears to be delayed compared to breaks in only one of the two homologues, since only bivalent breaks remain unrepaired at early pachytene, and are at mid and late pachytene the only aberration encountered (bivalents 1 and 3 being discontinuous in nuclei 3 and 78 respectively, while bivalent 5 was broken in the late pachytene nucleus 64 ). In Bombyx spermatocytes, all interlockings formed during zygotene are thus resolved, while a few (0.15-0.25 per nucleus) of the resultant bivalent breaks remain unrepaired at least until late pachytene. 

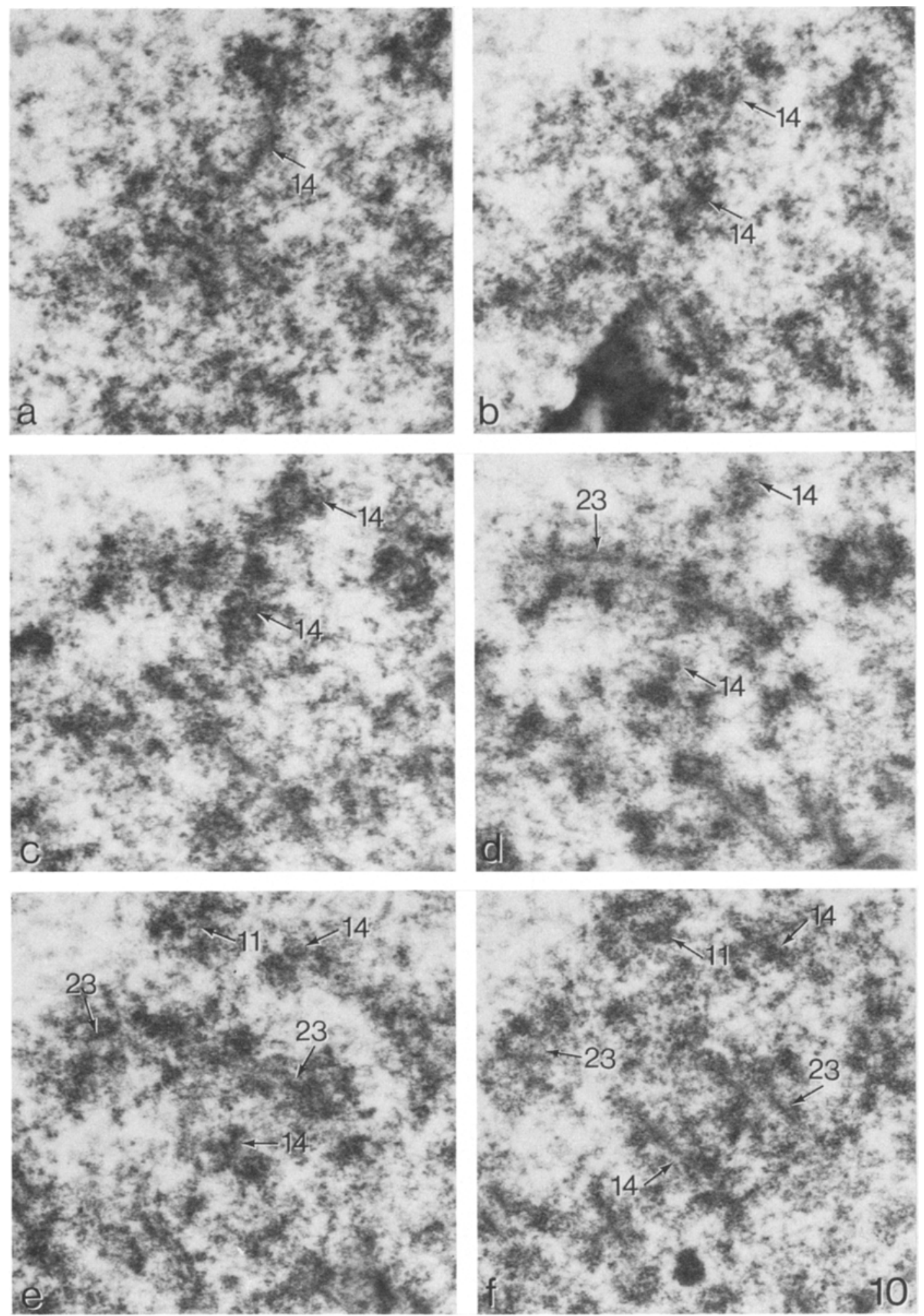

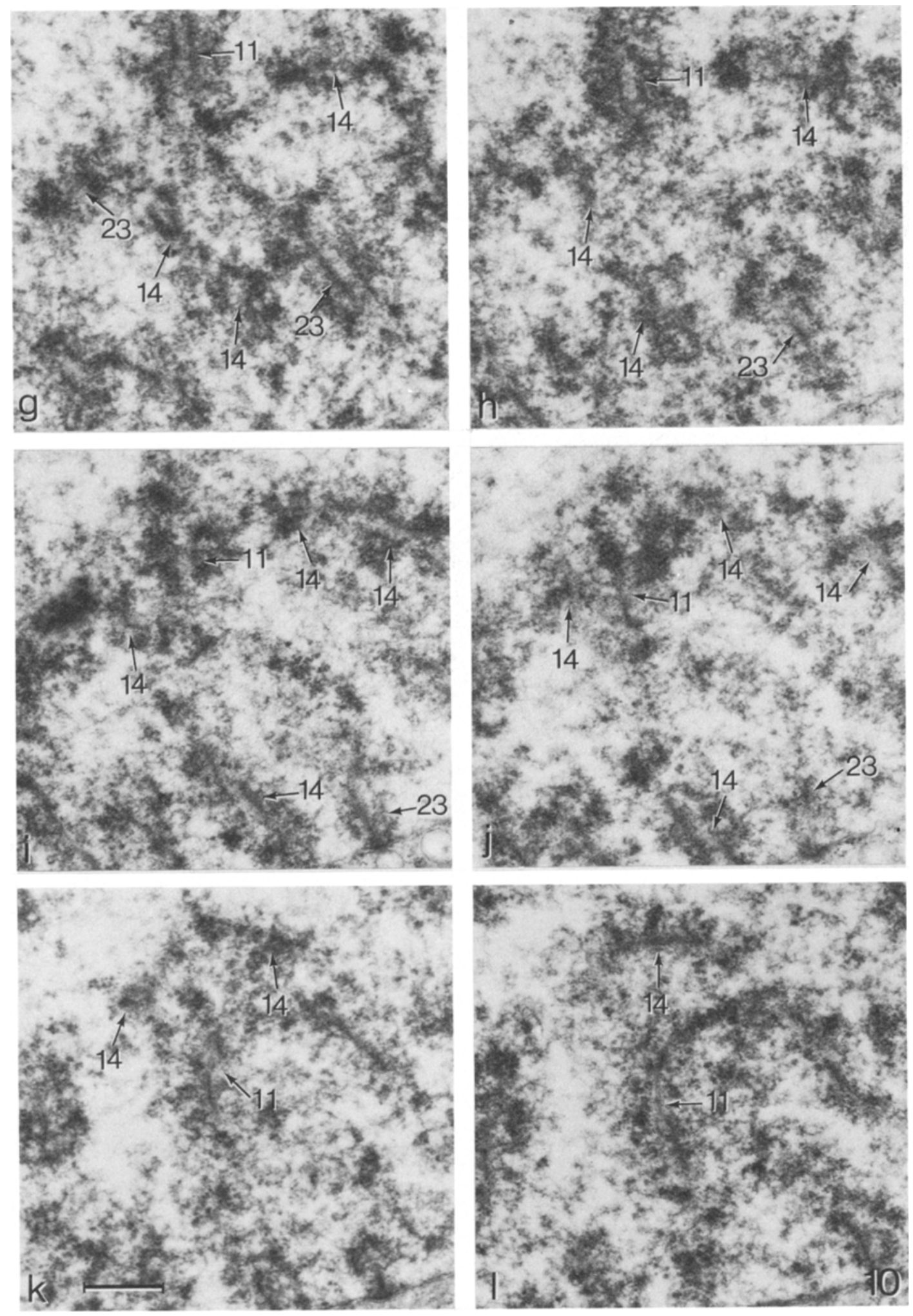
Figure 10. Twelve consecutive sections through a double interlocking at late zygotene (nucleus 21 ). Bivalents 11 and 23 are both interlocked by the unpaired segment of bivalent 14. A reconstruction of this interlocking is shown in Figure 11. (Bar $=0.5 \mu \mathrm{m})$.

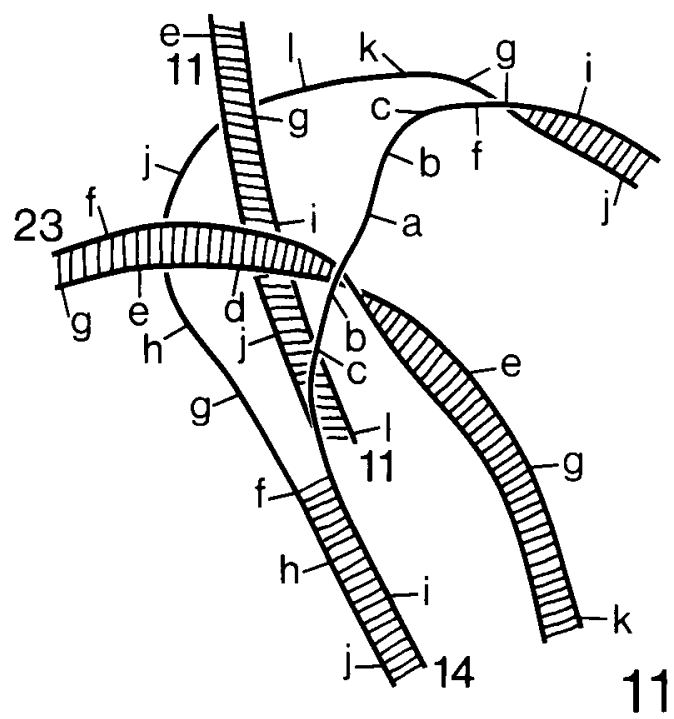

Figure 11. A reconstruction of the lateral components and synaptonemal complexes of the twelve consecutive sections in Figure 10. The letters refer to the micrographs in Figure 10.

\subsection{Mid pachytene (nuclei $1-4,78-81$ )}

The transition from early to mid pachytene is characterized by distinct changes in the ultrastructure of the cell: The two centriole pairs have migrated towards the cell membrane facing the lumen of the 64-cell cyst, and formation of the flagellae is initiated within an evagination of the cell membrane (Figure 22). As shown in Figure 21 , the distinct polarity of the nucleus seen at early pachytene is no longer apparent. In only some nuclei, is a reminiscence of the chromosome bouquet seen (Figure 23). The telomeres are still attached to the nuclear envelope through conical thickenings which often appear elongated. In some chromosome segments, one, or more rarely both lateral components are longitudinally divided (Figure 24). As described in detail in section 3.10., large and distinct recombination nodules are associated with the central region of the synaptonemal complex throughout this stage.
The total length of the synaptonemal complexes at mid pachytene is given in Table VI. The mean length amounts to $258 \pm 22 \mu \mathrm{m}$ with a variation from 234 to $295 \mu \mathrm{m}$.

\subsection{Late pachytene (nuclei $63,64,70-73$ )}

The polarity of the cytoplasm is no longer as prominent as at late zygotene and early pachytene but the two pairs of centrioles are still located near the cell membrane facing the lumen of the cyst (Figure 25).

The chromosome bouquet is at this stage completely resolved (Figure 25), but the telomeres remain attached to the nuclear envelope although the conical thickenings of the lateral components frequently appear stretched. The central component of the synaptonemal complex is more distinct and one or both lateral components are often longitudinally divided over shorter distances (Figure 24). Recombination nodules are present also at this stage together with more irregular nodules, both structures being associated with the central region of the complex (see section 3.10.). The chromatin frequently exhibits a chromomere-like pattern along the bivalents.

The total complement length varies from 208 to $328 \mu \mathrm{m}$ with a mean of $256 \pm 48 \mu \mathrm{m}$ (Table VII).

\subsection{Pachytene-diplotene}

The pachytene-diplotene transition is marked by the initiation of synaptonemal complex degradation accompanied by a progressive decondensation of the chromatin (Figure 26). The elimination of the synaptonemal complex appears to take place by a gradual degradation of its constituents, eventually resulting in synaptonemal complexes which are barely discernable. The elimination is not initiated synchronously along the bivalents but occurs in discrete, smaller segments while the intervening regions with more intact synaptonemal complexes frequently 
Table III

Description of the aberrations observed in the eight analyzed Bombyx spermatocyte nuclei at late zygotene.

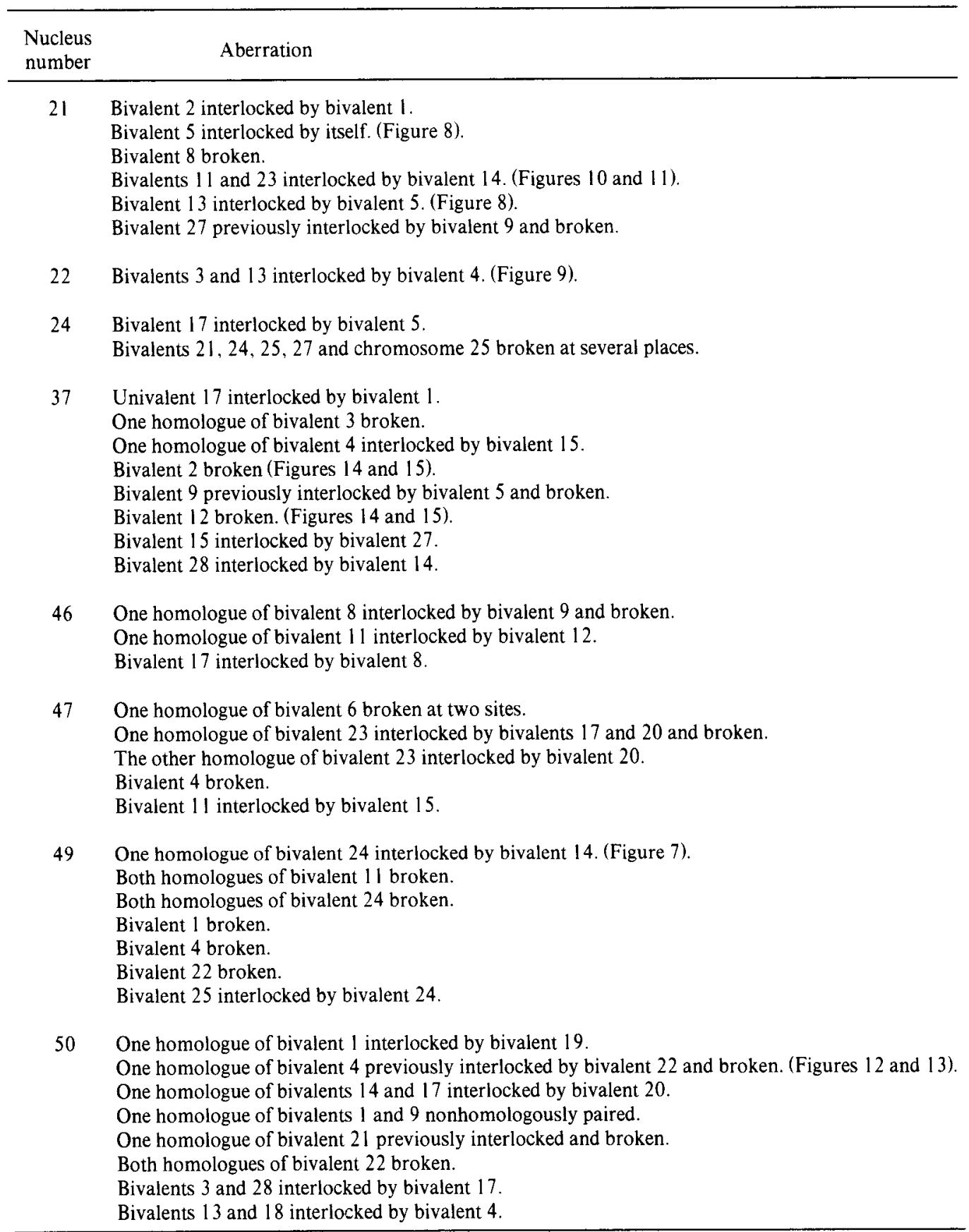

Figure 12. Eleven consecutive sections through a previously interlocked region at late zygotene (nucleus 50 ). Figure $12 \mathrm{~m}$ is a reconstruction of the lateral components and synaptonemal complexes of the eleven sections. One homologue of bivalent 4 has previously been interlocked by bivalent 22 . The interlocking is being resolved by breakage of the interlocked chromosome 4 and both chromosomes 22. (See Figure 13). (Bar $=0.5 \mu \mathrm{m}$ ). 

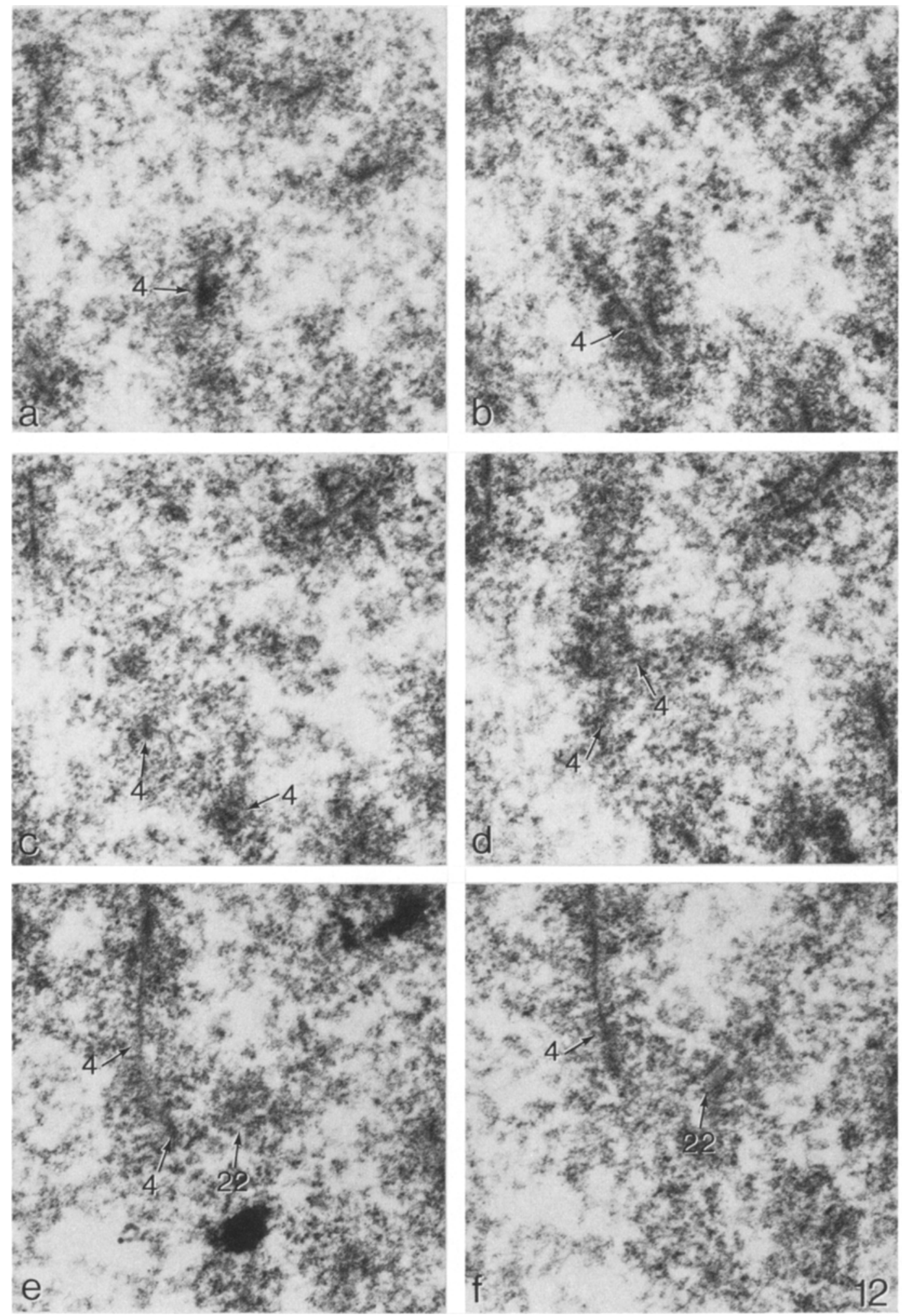

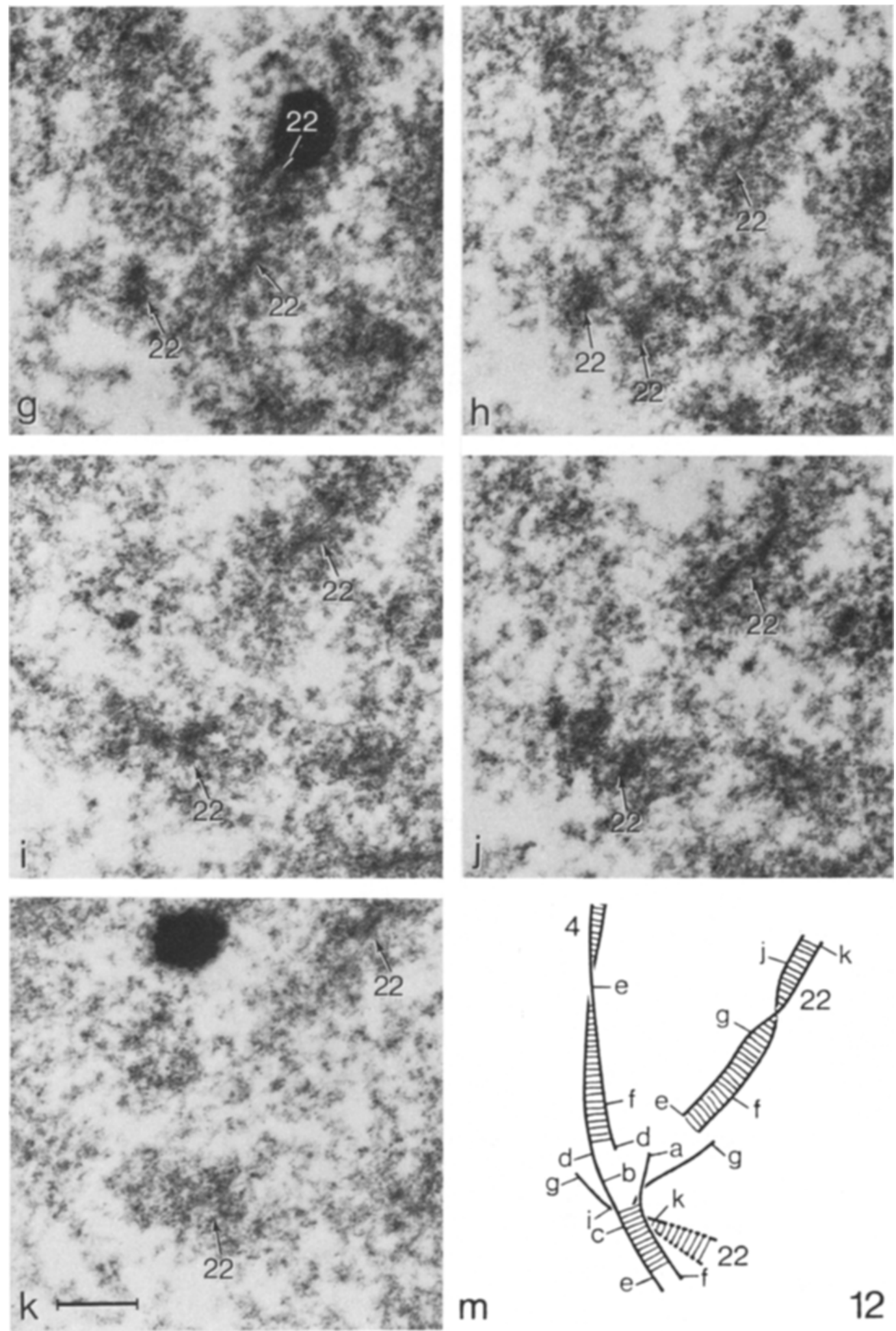

$\mathrm{m}$

12 
P. B. Holm \& S. W. Rasmussen: Meiosis in Bombyx males

Table IV

Interlockings and breaks in Bombyx spermatocyte nuclei at late zygotene.

\begin{tabular}{|c|c|c|c|c|c|c|}
\hline \multirow{2}{*}{$\begin{array}{l}\text { Nucleus } \\
\text { number }\end{array}$} & \multicolumn{2}{|c|}{ Interlockingsa) } & \multicolumn{3}{|c|}{ Breaks } & \multirow{2}{*}{$\begin{array}{l}\text { Total number of } \\
\text { aberrations }\end{array}$} \\
\hline & Chromosome & Bivalent & Chromosome & Bivalent & Total & \\
\hline 21 & 0 & 6 & 0 & 2 & 4 & 10 \\
\hline 22 & 0 & 2 & 0 & 0 & 0 & 2 \\
\hline 24 & 0 & 1 & - & - & - & (b) \\
\hline 37 & 2 & 3 & 1 & 3 & 7 & 12 \\
\hline 46 & 2 & 1 & 1 & 0 & 1 & 4 \\
\hline 47 & 3 & $i$ & 3 & 1 & 5 & 9 \\
\hline 49 & 1 & 1 & 4 & 3 & 10 & 12 \\
\hline 50 & 5 & 4 & 4 & 0 & 4 & 13 \\
\hline Mean & 1.6 & 2.4 & 1.6 & 1.1 & 3.8 & 7.9 \\
\hline
\end{tabular}

a) The values include resolving interlockings.

b) Several breaks in 5 pairs of homologues, see Table III.

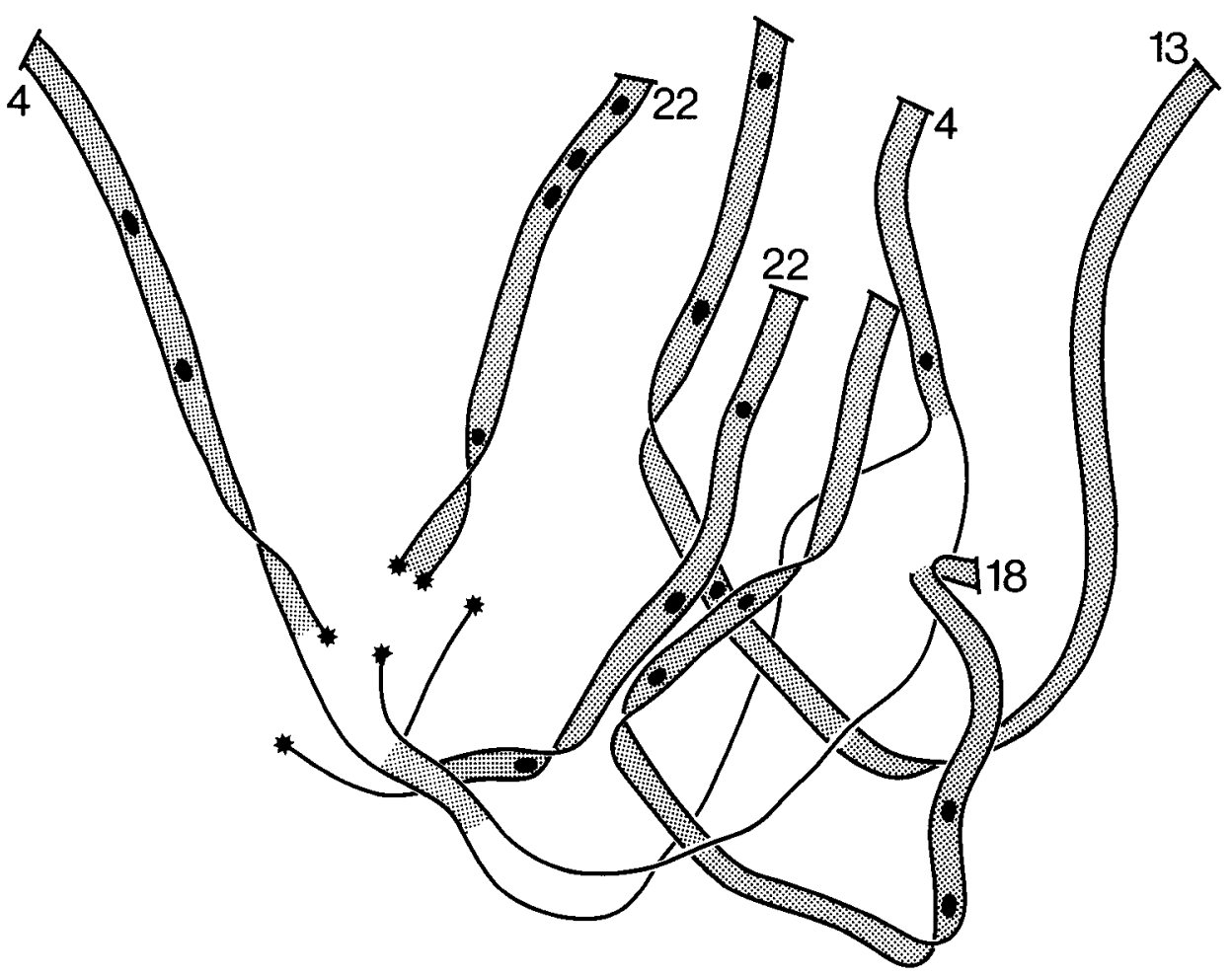

Figure 13. A partial reconstruction of a double bivalent interlocking and a resolving chromosome interlocking at late zygotene (nucleus 50). The broken ends are marked by stars. (See Figure 12). 

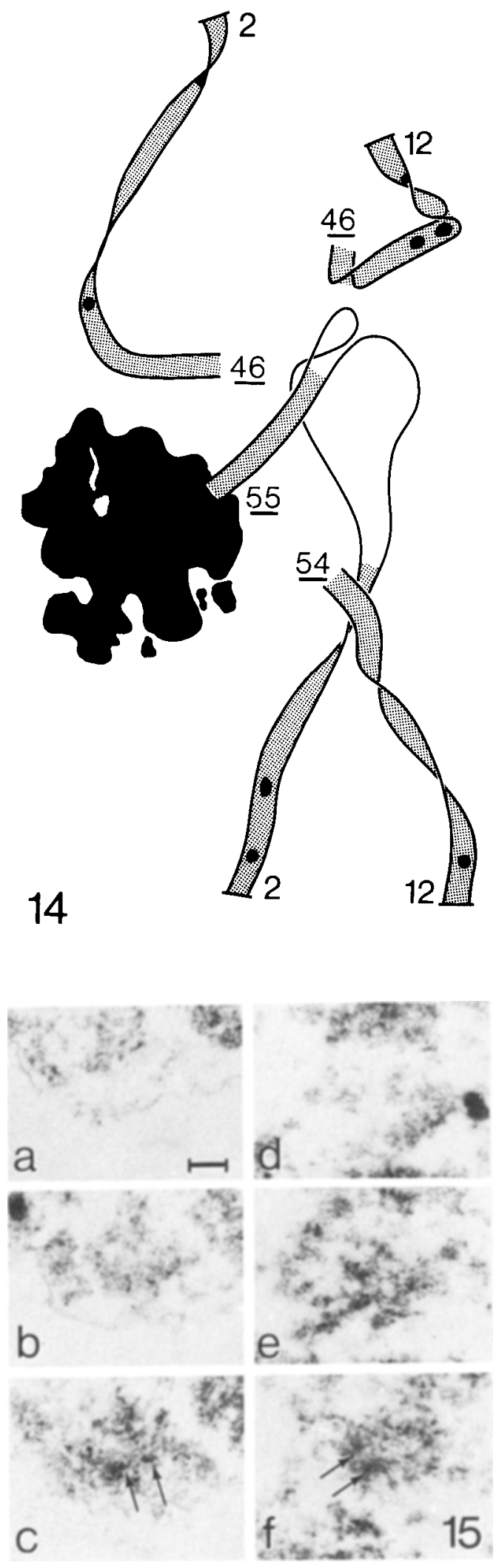

Figures 14 and 15. Partial reconstruction of broken bivalents at late zygotene (nucleus 37 ). Note the considerable distance separating the broken ends. For bivalent 12, the distance amounts to $2.4 \mu \mathrm{m}$ and for bivalent 2 to $1.3 \mu \mathrm{m}$. The broken ends of bivalent 12 are shown in consecutive sections in Figure 15. Figures $15 a-c$ show the upper broken end of bivalent 12 and Figures $15 \mathrm{~d}-\mathrm{f}$ the lower end. (Arrows denote lateral components). The underlined numbers are the section numbers. (Bar $=0.5 \mu \mathrm{m})$.

possess double lateral components and structurally modified central regions. In one of the four nuclei (number 42), synaptonemal complex degradation had progressed too far to allow a reliable reconstruction of its synaptonemal complex complement while in the remaining three nuclei (numbers 44,61 and 62), complete reconstructions were performed. Although the degradation of the complex was in progress in the individual bivalents the synaptonemal complexes in the reconstruction (Figure 27) are drawn with the same signature as used in the previous stages. When compared to mid pachytene and late pachytene (Figure 23) it is evident that the bivalents at the pachytene - diplotene transition are considerably coiled and twisted.

The change in the path of the bivalents and the alteration of the structure of the synaptonemal complex are accompanied by a substantial increase in the length of the complement. As shown in Table VIII, the mean length now amounts to $347 \pm 45 \mu \mathrm{m}$ with a range from 296 to $374 \mu \mathrm{m}$.

\subsection{Recombination nodules and chromatin nodules}

Recombination nodules are observed from the beginning of zygotene through pachytene and are associated with the central region of the synaptonemal complex as described in the previous sections. Throughout zygotene, the nodules vary in shape from spheres to ellipsoids or dumbbells, the shorter diameter ranging from $30-60 \mathrm{~nm}$ and the longer from $30-100 \mathrm{~nm}$ (Figures 28ab). As intermediate forms between smaller and larger nodules are frequent, attempts to distinguish different types of nodules have failed. The electron density of the nodules varies from that 


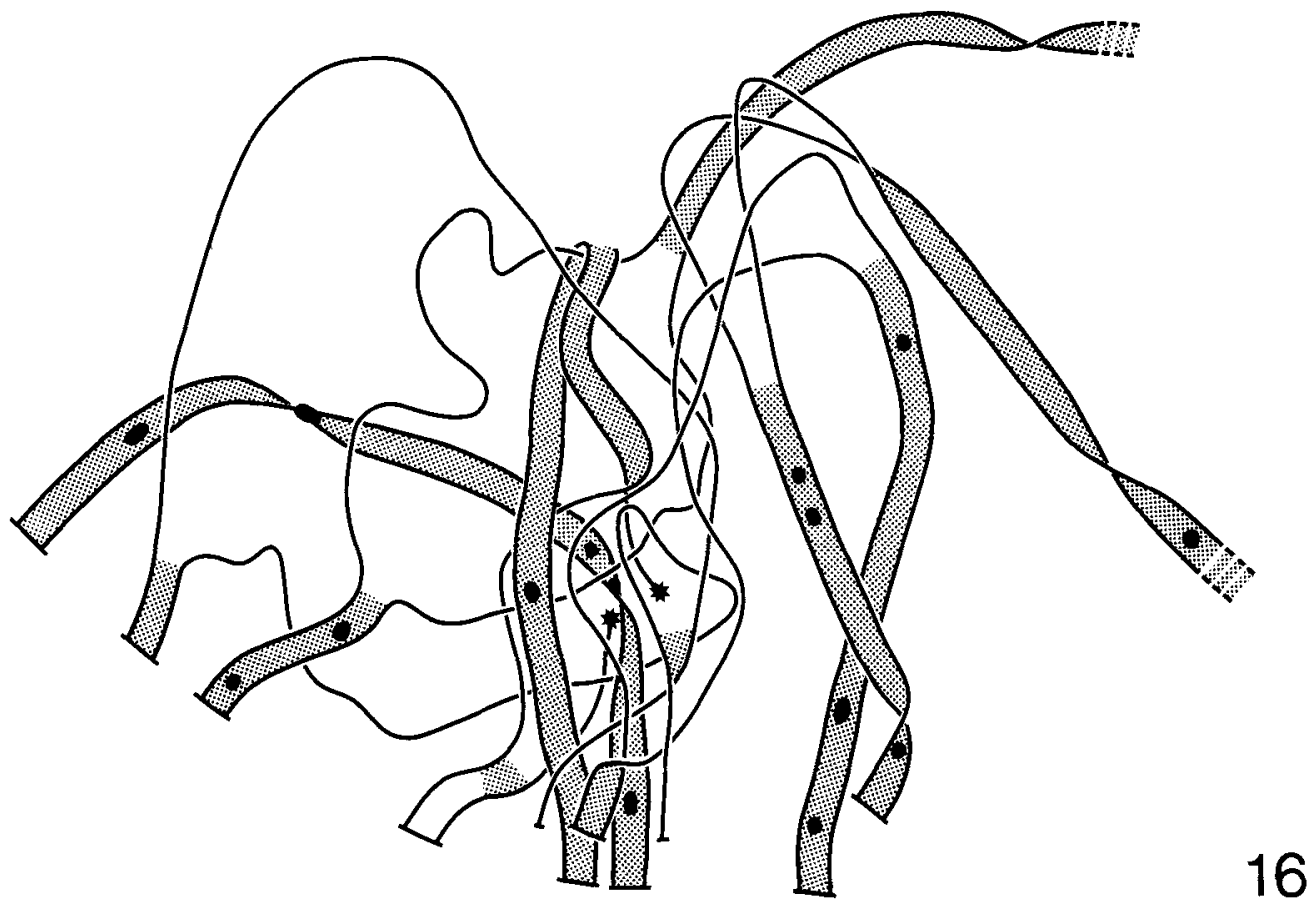

Figure 16. Complex interlocking of six bivalents at late zygotene (nucleus 50). Ends of broken bivalents are marked by stars.

\section{Table V}

Synaptonemal complex (SC) length and numbers of recombination nodules $(\mathrm{RN})$ and chromatin nodules $(\mathrm{CN})$ in Bombyx spermatocytes at early pachytene.

\begin{tabular}{ccccccc}
\hline $\begin{array}{c}\text { Nucleus } \\
\text { number }\end{array}$ & $\begin{array}{c}\text { SC length } \\
(\mu \mathrm{m})\end{array}$ & $\begin{array}{c}\text { Number } \\
\text { of RN }\end{array}$ & $\begin{array}{c}\text { Number } \\
\text { of CN }\end{array}$ & $\begin{array}{c}\text { Total number } \\
\text { of nodules }\end{array}$ & $\begin{array}{c}\text { SC length per } \\
\text { nodule }(\mu \mathrm{m})\end{array}$ & $\begin{array}{c}\text { Number of } \\
\text { bivalents } \\
\text { without } \\
\text { nodules }\end{array}$ \\
\hline 5 & 207 & 30 & 6 & 36 & 5.8 & 7 \\
6 & 214 & 51 & 0 & 51 & 4.2 & 2 \\
7 & 185 & 48 & 0 & 48 & 3.9 & 3 \\
8 & 201 & 46 & 9 & 55 & 3.7 & 0 \\
9 & 195 & 35 & 6 & 41 & 4.7 & 6 \\
10 & 194 & 50 & 0 & 50 & 3.9 & 2 \\
13 & 209 & 80 & 0 & 80 & 2.6 & 2 \\
14 & 184 & 68 & 0 & 68 & 2.7 & 1 \\
15 & 208 & 75 & 0 & 75 & 2.8 & 3 \\
16 & 183 & 46 & 5 & 51 & 3.6 & 1 \\
23 & 182 & 53 & 1 & 54 & 3.4 & 1 \\
25 & 200 & 70 & 1 & 71 & 2.8 & 2 \\
26 & 203 & 61 & 5 & 66 & 3.1 & 1 \\
27 & 199 & 71 & 2 & 73 & 2.7 & 2.8 \\
28 & 201 & 72 & 0 & 72 & 2.6 & 2 \\
48 & 203 & 78 & 0 & 78 & 3.2 & \\
\hline Mean \pm s.d. & $198 \pm 10$ & $58 \pm 15$ & $2 \pm 3$ & $61 \pm 14$ & & \\
\hline
\end{tabular}




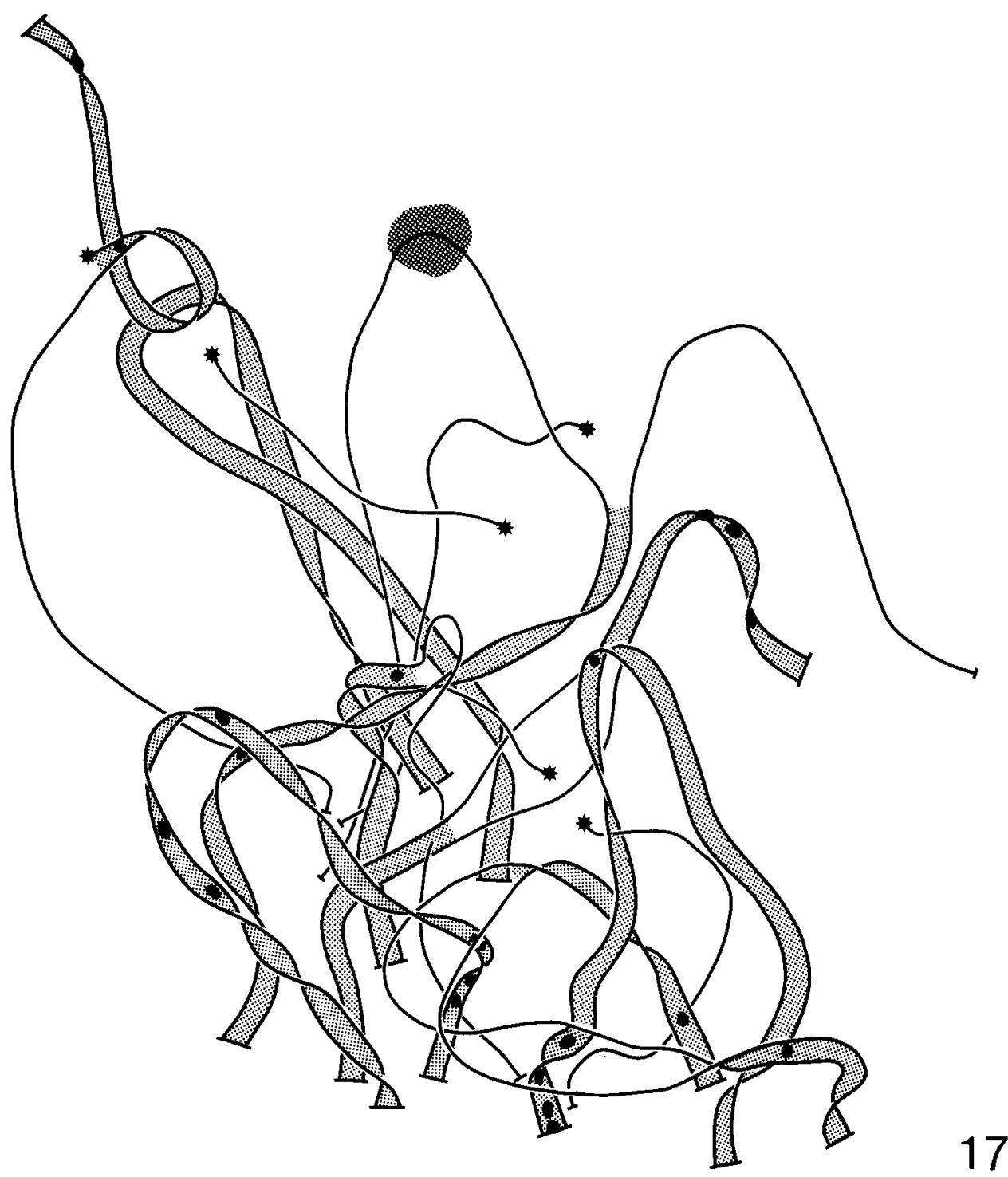

Figure 17. Complex interlocking involving eight bivalents at late zygotene (nucleus 47). Three chromosome breaks have given rise to six free chromosome ends (marked by stars).

of the chromatin to a density similar to that of the lateral components. In most cases, recombination nodules are connected to the lateral components through fine filaments and are either located within or more frequently slightly lateral to the central region of the complex (Figure 28b).

At early and mid pachytene, several of the nodules appear larger and more electron dense and elongated than those present at late xygotene (Figures $28 \mathrm{c}-\mathrm{h}$ ) and the position of this type is always lateral to the central component. In few cases, elongated nodules form cross-bridges at different angles between the lateral components. Several nodules of the smaller type seen at earlier stages are also present together with a number 


\section{Table VI}

Synaptonemal complex (SC) length and numbers of recombination nodules (RN) and chromatin nodules $(\mathrm{CN})$ in Bombyx spermatocytes at mid pachytene.

\begin{tabular}{ccccccc}
\hline $\begin{array}{c}\text { Nucleus } \\
\text { number }\end{array}$ & $\begin{array}{c}\text { SC length } \\
(\mu \mathrm{m})\end{array}$ & $\begin{array}{c}\text { Number } \\
\text { of RN }\end{array}$ & $\begin{array}{c}\text { Number } \\
\text { of CN }\end{array}$ & $\begin{array}{c}\text { Total number } \\
\text { of nodules }\end{array}$ & $\begin{array}{c}\text { SC length per } \\
\text { nodule }(\mu \mathrm{m})\end{array}$ & $\begin{array}{c}\text { Number of } \\
\text { bivalents } \\
\text { without } \\
\text { nodules }\end{array}$ \\
\hline 1 & 271 & 34 & 23 & 57 & 3.8 & 0 \\
2 & 295 & 31 & 24 & 55 & 5.4 & 0 \\
3 & 273 & 33 & 27 & 60 & 4.6 & 0 \\
4 & 266 & 29 & 17 & 46 & 5.8 & 1 \\
78 & 238 & 33 & 27 & 60 & 4.0 & 1 \\
79 & 234 & 59 & 3 & 62 & 3.8 & 0 \\
80 & 243 & 38 & 3 & 41 & 5.9 & 1 \\
81 & 245 & 42 & 20 & 62 & 4.0 & 2 \\
\hline Mean \pm s.d. & $258 \pm 22$ & $37 \pm 9$ & $18 \pm 10$ & $55 \pm 8$ & 4.6 & 0.6 \\
\hline
\end{tabular}

Table VII

Synaptonemal complex (SC) length and numbers of recombination nodules (RN) and chromatin nodules (CN) in Bombyx spermatocytes at late pachytene.

\begin{tabular}{ccccccc}
\hline $\begin{array}{c}\text { Nucleus } \\
\text { number }\end{array}$ & $\begin{array}{c}\text { SC length } \\
(\mu \mathrm{m})\end{array}$ & $\begin{array}{c}\text { Number } \\
\text { of RN }\end{array}$ & $\begin{array}{c}\text { Number } \\
\text { of CN }\end{array}$ & $\begin{array}{c}\text { Total number } \\
\text { of nodules }\end{array}$ & $\begin{array}{c}\text { SC length per } \\
\text { nodule }(\mu \mathrm{m})\end{array}$ & $\begin{array}{c}\text { Number of } \\
\text { bivalents } \\
\text { without } \\
\text { nodules }\end{array}$ \\
\hline 63 & 328 & 31 & 43 & 74 & 4.2 & 1 \\
64 & 304 & 14 & 54 & 68 & 4.5 & 0 \\
70 & 208 & 27 & 51 & 78 & 2.7 & 1 \\
71 & 235 & 32 & 26 & 58 & 4.1 & 0 \\
72 & 224 & 35 & 36 & 71 & 3.1 & 0 \\
73 & 240 & 19 & 51 & 70 & 3.4 & 1 \\
\hline Mean \pm s.d. & $256 \pm 48$ & $26 \pm 7$ & $44 \pm 11$ & $70 \pm 7$ & 3.7 & 0.5 \\
\hline
\end{tabular}

\section{Table VIII}

Synaptonemal complex (SC) length and numbers of recombination nodules (RN) and chromatin nodules $(\mathrm{CN})$ in Bombyx spermatocytes at the transition from pachytene to diplotene.

\begin{tabular}{ccccccc}
\hline $\begin{array}{c}\text { Nucleus } \\
\text { number }\end{array}$ & $\begin{array}{c}\text { SC length } \\
(\mu \mathrm{m})\end{array}$ & $\begin{array}{c}\text { Number } \\
\text { of RN }\end{array}$ & $\begin{array}{c}\text { Number } \\
\text { of CN }\end{array}$ & $\begin{array}{c}\text { Total number } \\
\text { of nodules }\end{array}$ & $\begin{array}{c}\text { SC length per } \\
\text { nodule }(\mu \mathrm{m})\end{array}$ & $\begin{array}{c}\text { Number of } \\
\text { bivalents } \\
\text { without } \\
\text { nodules }\end{array}$ \\
\hline 44 & 296 & 8 & 53 & 61 & 4.8 & 1 \\
61 & 373 & 23 & 68 & 91 & 4.1 & 0 \\
62 & 374 & 18 & 56 & 74 & 5.0 & 2 \\
\hline Mean \pm s.d. & $347 \pm 45$ & $16 \pm 6$ & $59 \pm 8$ & $75 \pm 15$ & 4.6 & 1 \\
\hline
\end{tabular}


Table IX

Mean synaptonemal complex (SC) length and numbers of recombination nodules (RN) and chromatin nodules (CN) from late zygotene to the pachytene - diplotene transition in Bombyx spermatocytes.

\begin{tabular}{lccccccc}
\hline Stage & $\begin{array}{c}\text { Number of } \\
\text { nuclei }\end{array}$ & $\begin{array}{c}\text { SC length } \\
(\mu \mathrm{m} \pm \mathrm{s.d})\end{array}$ & $\begin{array}{c}\text { Number } \\
\text { of RN }\end{array}$ & $\begin{array}{c}\text { Number } \\
\text { of CN }\end{array}$ & $\begin{array}{c}\text { Total } \\
\text { number of } \\
\text { nodules }\end{array}$ & $\begin{array}{c}\text { SC length } \\
\text { per nodule } \\
(\mu \mathrm{m})\end{array}$ & $\begin{array}{c}\text { Percent } \\
\text { bivalents } \\
\text { without } \\
\text { nodules }\end{array}$ \\
\hline Late zygotene & 8 & $180 \pm 17 \mathrm{a})$ & $91 \pm 14$ & 0 & $103 \pm 21 \mathrm{~b})$ & 2.0 & 3.1 \\
Early pachytene & 16 & $198 \pm 10$ & $58 \pm 15$ & $2 \pm 3$ & $61 \pm 14$ & 3.2 & 7.8 \\
Mid pachytene & 8 & $258 \pm 22$ & $37 \pm 9$ & $18 \pm 10$ & $55 \pm 8$ & 4.6 & 2.2 \\
Late pachytene & 6 & $256 \pm 48$ & $26 \pm 7$ & $44 \pm 11$ & $70 \pm 7$ & 3.7 & 1.8 \\
Pachytene-diplotene & 3 & $347 \pm 45$ & $16 \pm 6$ & $59 \pm 8$ & $75 \pm 15$ & 4.6 & 3.6 \\
\hline
\end{tabular}

a) A total synaptonemal complex length of $202 \pm 17 \mu \mathrm{m}$ is expected upon completion of pairing.

b) The number expected upon completion of synaptonemal complex formation.

\section{Table X}

The number of recombination nodules ( $R N)$, chromatin nodules $(\mathrm{CN})$ and chiasmata during late meiotic prophase in Bombyx spermatocytes.

\begin{tabular}{|c|c|c|c|c|}
\hline Stage & Nucleus number & $\begin{array}{l}\text { Number of } \mathrm{CN} \\
\text { and chiasmata }\end{array}$ & Mean \pm s.d. & Number of RN \\
\hline Early diplotene & $\begin{array}{l}17 \\
18 \\
19 \\
20\end{array}$ & $\begin{array}{l}59 \\
51 \\
67 \\
61\end{array}$ & $60 \pm 7$ & $\begin{array}{l}1 \\
3 \\
5 \\
0\end{array}$ \\
\hline Mid diplotene & $\begin{array}{l}41 \\
43 \\
45 \\
65 \\
67 \\
68\end{array}$ & $\begin{array}{l}56 \\
59 \\
63 \\
56 \\
67 \\
73\end{array}$ & $62 \pm 7$ & $\begin{array}{l}0 \\
0 \\
0 \\
0 \\
2 \\
0\end{array}$ \\
\hline Late diplotene & $\begin{array}{l}51 \\
52\end{array}$ & $\begin{array}{l}42 \\
50\end{array}$ & 46 & $\begin{array}{l}0 \\
0\end{array}$ \\
\hline Diplotene-diakinesis & $\begin{array}{l}53 \\
54 \\
55 \\
56\end{array}$ & $\begin{array}{l}53 \\
53 \\
51 \\
60\end{array}$ & $55 \pm 4$ & $\begin{array}{l}0 \\
0 \\
0 \\
0\end{array}$ \\
\hline Early diakinesis & 59 & 58 & 58 & 0 \\
\hline Mid diakinesis & $\begin{array}{l}58 \\
60 \\
61 \mathrm{a}\end{array}$ & $\begin{array}{l}53 \\
70 \\
62\end{array}$ & $62 \pm 9$ & $\begin{array}{l}0 \\
0 \\
0\end{array}$ \\
\hline Late diakinesis & 29 & 28 bivalents & & \\
\hline Metaphase I & $\begin{array}{l}33 \\
34 \\
35 \\
36 \\
75\end{array}$ & 28 bivalents & & \\
\hline
\end{tabular}




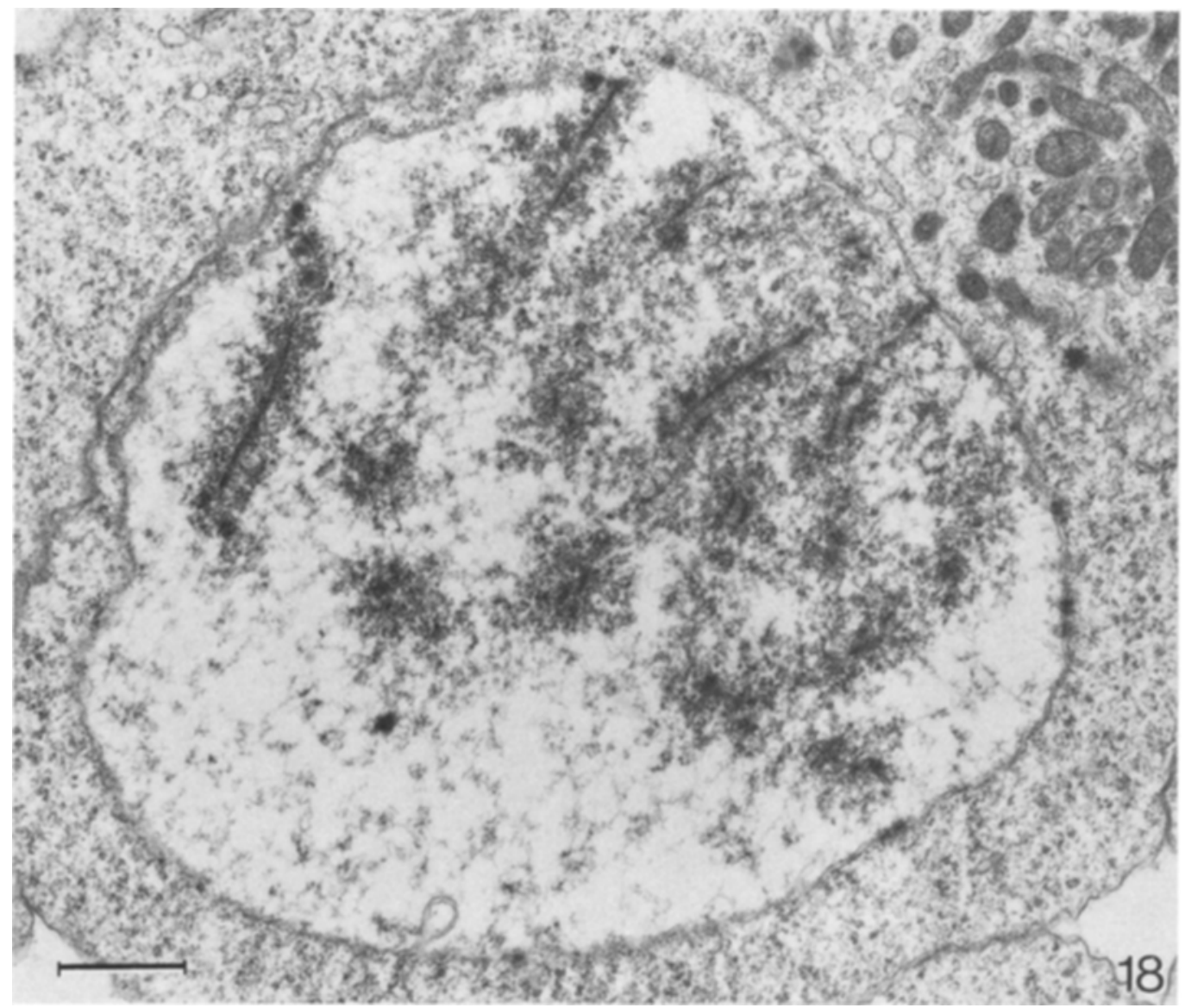

Figure 18. Survey micrograph of an early pachytene nucleus. $($ Bar $=1.0 \mu \mathrm{m})$.

of intermediate forms, and at this stage it is not possible to distinguish separate classes of nodules.

Some of the large, dense recombination nodules increase in size at mid pachytene by addition of material resembling chromatin to the surface of the nodules, giving the structure an irregular appearance (Figures 29a-d). This chromatin - recombination nodule aggregate, hereafter termed chromatin nodule, remains associated with the central region of the synaptonemal complex. The evolution of chromatin nodules appears to be a continuous process, as intermediates between recombination nodules and chromatin nodules are frequent and it is often not possible to discriminate unequivocally between the two forms.

The chromatin nodules increase in size as well

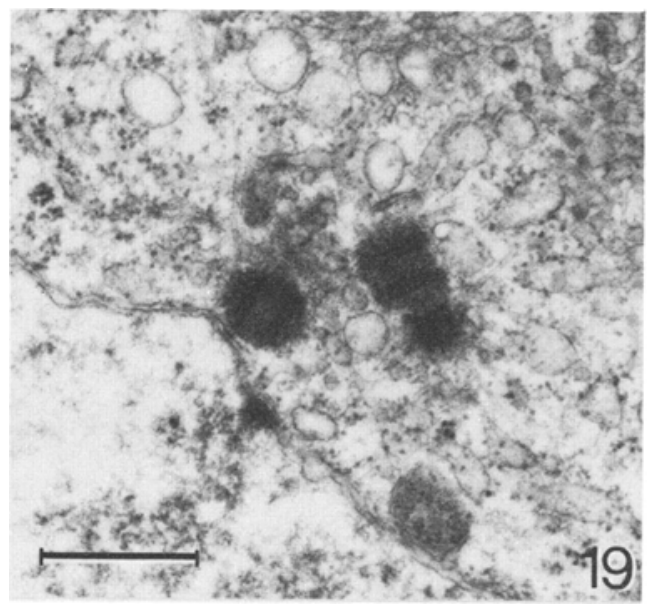

Figure 19. Centrioles at early pachytene. At this stage the two pairs of centrioles are located near the nuclear envelope outside the bouquet region. $(\mathrm{Bar}=0.5 \mu \mathrm{m})$. 


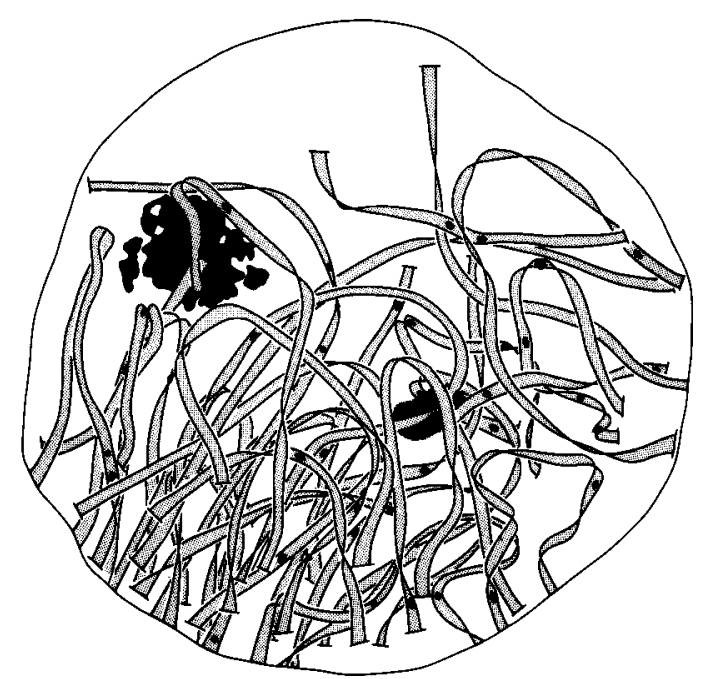

Figure 20. Reconstruction of an early pachytene nucleus (number 16). (Magnification approximately 9,000 times).

as in number during late pachytene (Figures $29 \mathrm{a}-\mathrm{b})$ and at the pachytene-diplotene transition most nodules are of this type (Figures $29 \mathrm{c}-\mathrm{d}$ ). Simultaneously, recombination nodules become less frequent and by early diplotene only few remain.

\subsection{Early diplotene (nuclei 17-20)}

The most pronounced morphological characteristic of early diplotene is the absence of a recognizable bivalent continuity: the unraveling of the chromatin and the decomposition of synaptonemal complexes which are initiated during the pachytene - diplotene transition have now progressed too far to permit the tracing of entire bivalents either by their chromatin contour or by their synaptonemal complexes (Figure 30). Only shorter stretches of still continuous synaptonemal complex and diverging lateral components can be followed at this stage. Such reconstructions (Figure 31) reveal that the elimination of the synaptonemal complex often involves a longitudinal separation of individual lateral components into two distinct subunits. It is, however, not possible to determine whether this phenomenon is accompanied by a separation also of the two sister chromatids or whether the splitting of the lateral components occurs after their detachment from the chromatin.

As shown in Figure 31, chromatin nodules are frequent at this stage and are associated with the central region of more or less intact pieces of synaptonemal complex (see Table $\mathrm{X}$ and section 3.19.2.), which also occasionally contain recombination nodules (Figure 31). During early diplotene, chromatin nodules gradually increase in size by differential chromatin condensation around the nodule (Figures $29 \mathrm{e}-\mathrm{f}$ ).

\subsection{Mid diplotene (nuclei $41,43,45,65,67$, 68)}

At mid diplotene the nucleus moves towards the cell membrane and the proximal end of the centrioles associates with the outer membrane of the nuclear envelope (Figures 33 and 34). Chromatin decondensation reaches its maximum at this stage and the continued degradation of the synaptonemal complex has left only short and poorly defined stretches of synaptonemal complex (Figure 32). Only about 60 major (Table X) and a few minor chromatin condensations remain, the major condensations in most cases being associated with remnants of synaptonemal complexes, partly surrounding the complex and 


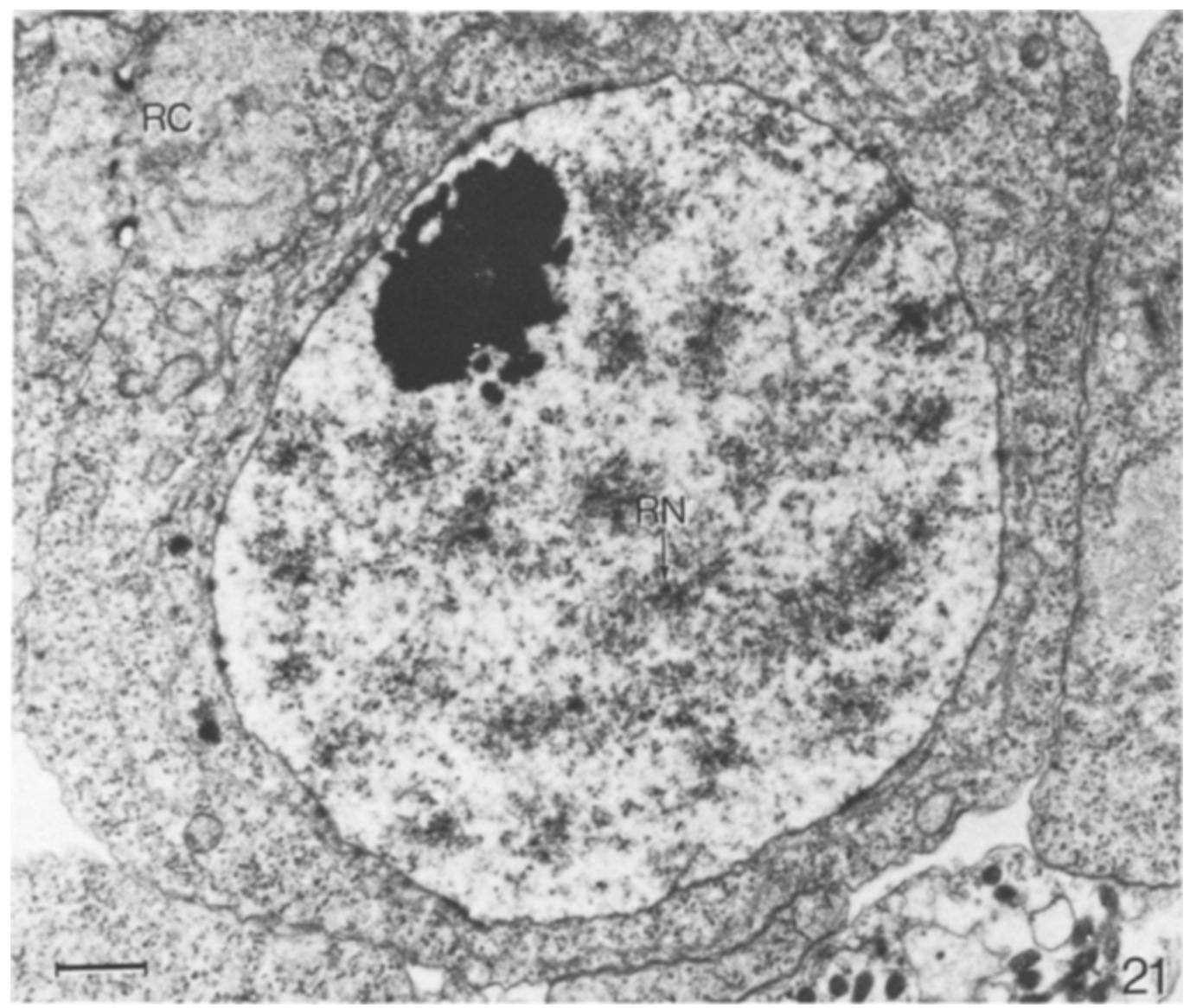

Figure 21. Survey micrograph of a mid pachytene nucleus. RN, recombination nodule; $\mathrm{RC}$, ring canal. (Bar = $1.0 \mu \mathrm{m})$.

filling out its central region (see Figures $29 \mathrm{~g}-\mathrm{h}$ and 34). Often these domains contain a dense core reminiscent of a recombination nodule. As judged by the presence of intermediate forms the condensations have evolved from chromatin nodules and in combination with the synaptonemal complex fragments they constitute early chiasmata.

Figure 22. Centrioles at mid pachytene. The two pairs of centrioles have at this stage migrated to the cell membrane and the growth of the flagellae has started. $($ Bar $=0.5 \mu \mathrm{m})$.

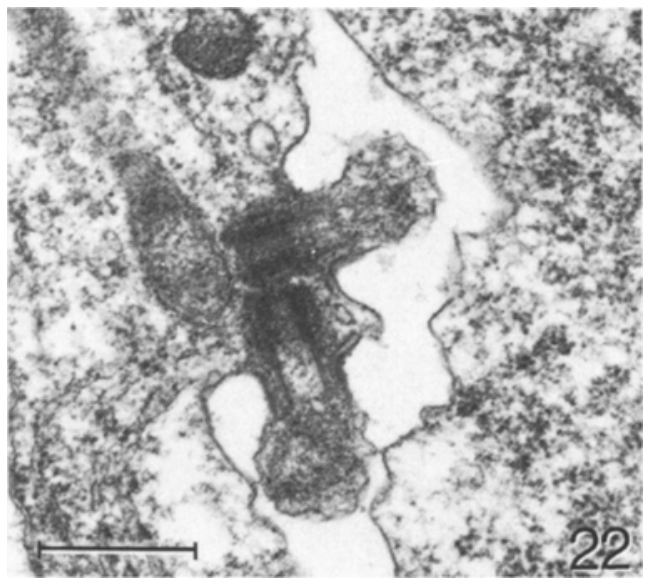




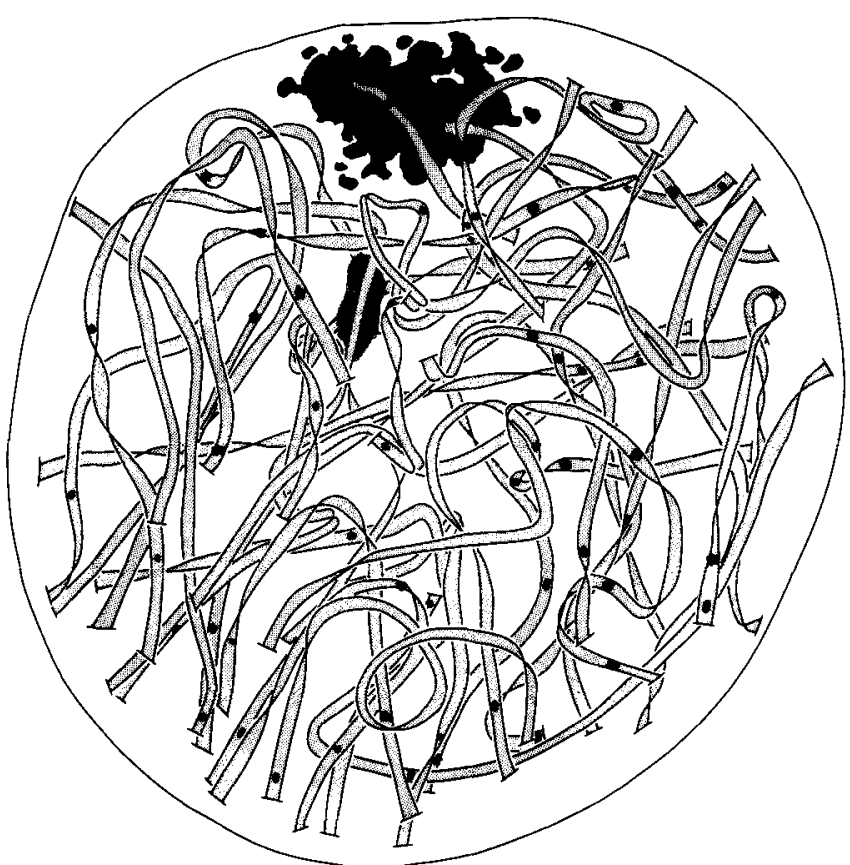

Figure 23. Reconstruction of a mid pachytene nucleus (number 1). (Magnification approximately 9,000 times).

\subsection{Late diplotene (nuclei 51,52 )}

During late diplotene, the centrioles and the nuclear envelope remain associated as illustrated in Figures 35 and 36. Elimination of the synaptonemal complexes is almost complete with only a few remnants of the lateral components remaining (Figure 37). As in the previous two stages, the bulk of the chromatin is decondensed and individual bivalents cannot be distinguished (Figure 35).

At this stage the major chromatin condensations consist of a central, circular component $120-160 \mathrm{~nm}$ in diameter, often associated with remnants of lateral components and always flanked by two domains of condensed chromatin (Figure 37). High magnifications of the circular component (Figure 38) reveal a dense, central core, a number of radiating filaments and an outer ring with an electron density similar to that of the chromatin (see Figure 38b for a simple diagrammatic representation ). The whole structure is intimately associated with the condensed chromatin, and it is apparent that the structure represents a further evolution of the major chromatin condensations present at mid diplotene and hence constitutes a more advanced chiasma.

\subsection{Diplotene-diakinesis (nuclei 53-56)}

The survey michrograph of a nucleus at the transition from diplotene to diakinesis in Figure 39 and the partial reconstruction shown in Figure 40 illustrate the morphological characteristics of this stage, namely the nearly complete elimination of the lateral components, and the initiation of chromatin recondensation. The latter process appears to occur in a non-uniform fashion along the individual bivalents giving rise to an increase in the number of differentially condensed chromatin regions with intervening regions of dispersed chromatin.

Chiasmata are at this stage distinctly tripartite consisting of two chromatin regions combined by a chromatin bridge with its circular component (Figure 41). Outside such regions, the continuity of the chromatin is hardly recognizable (Figure $4 \mathrm{lg}-\mathrm{i}$ ). The high magnification of the 

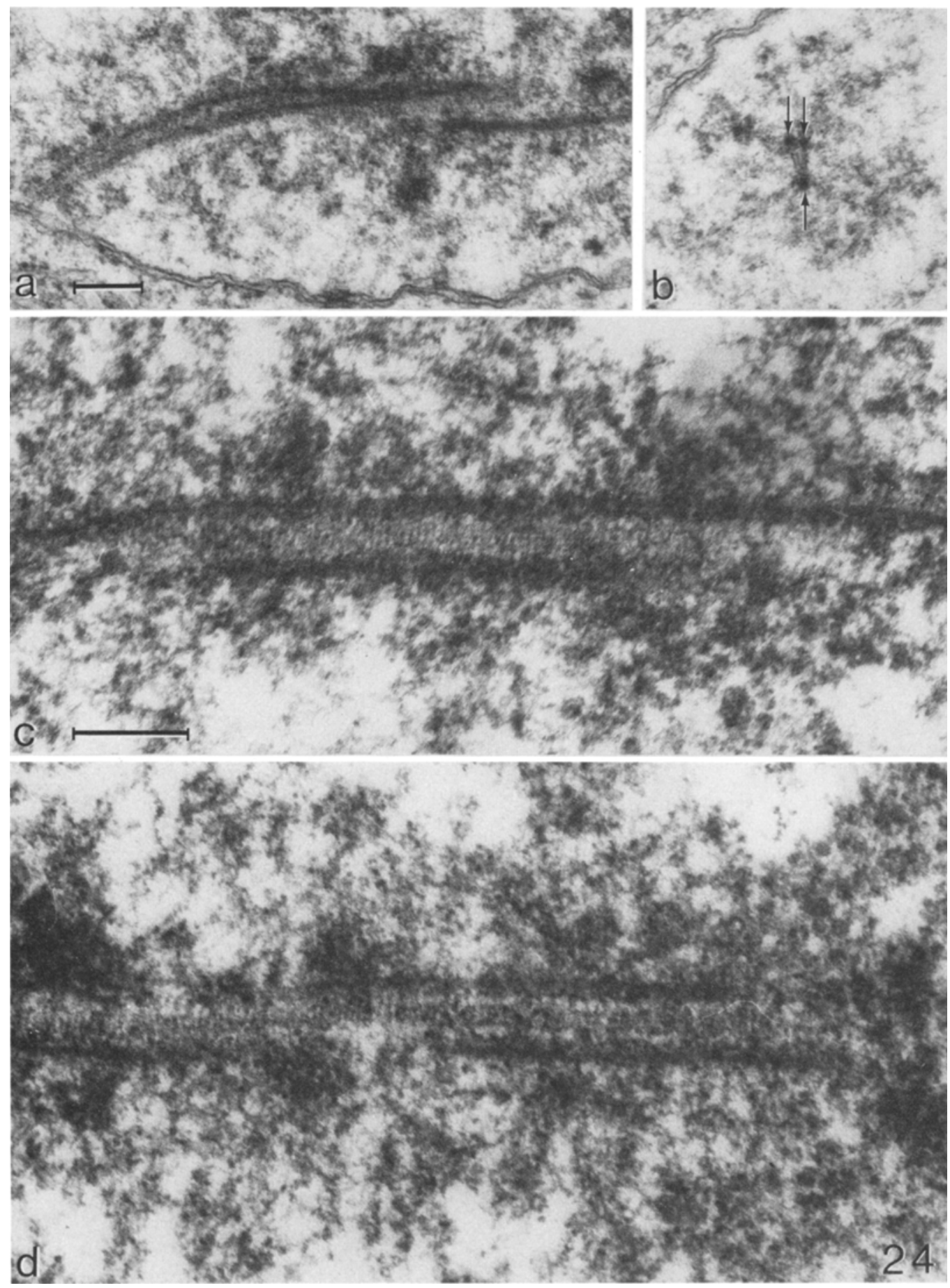

Figure 24. Higher magnifications of synaptonemal complexes at mid and late pachytene. Figure 24a and b show double lateral components at mid pachytene. Note that only one of the lateral components (denoted by arrows) are double in Figure 24b. Figures $24 \mathrm{c}$ and $\mathrm{d}$ are longitudinal sections of synaptonemal complexes at mid and late pachytene respectively. $($ Bar $=0.2 \mu \mathrm{m})$. 


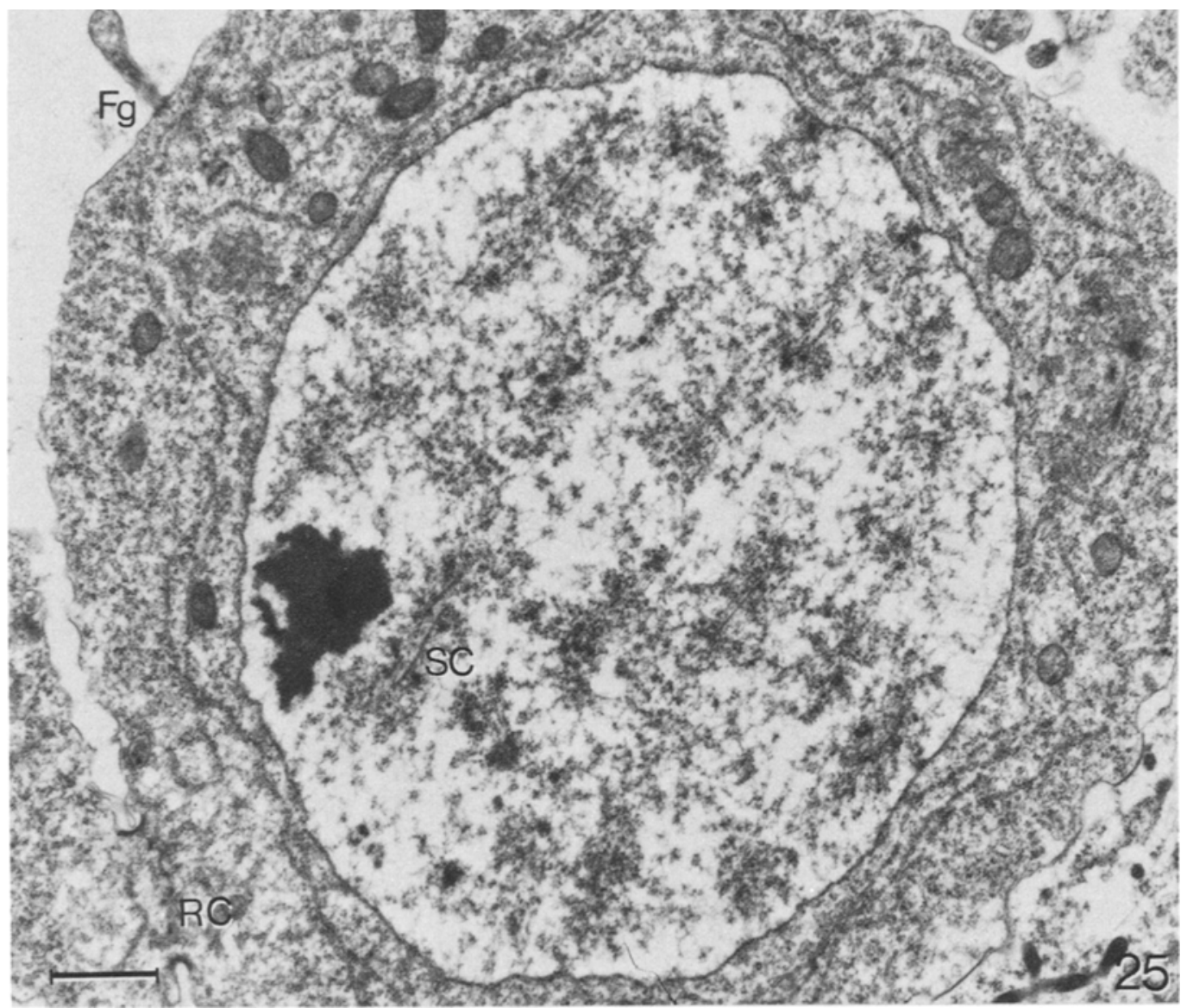

Figure 25. Survey micrograph of a late pachytene nucleus. SC, synaptonemal complex; RC, ring canal; Fg, flagellum. $(\operatorname{Bar}=1.0 \mu \mathrm{m})$.

circular component of the chiasma in Figure 42 shows that the structure consists of a dense core approximately $20 \mathrm{~nm}$ in diameter, an inner and an outer ring with diameters of about 60 and
$160 \mathrm{~nm}$ respectively. The latter appears to be composed of discrete, dense units. A number of filaments connect the inner and the outer ring. As in late diplotene, the surrounding chromatin

Figure 26. Survey micrograph of a nucleus at the transition from pachytene to diplotene. $($ Bar $=1.0 \mu \mathrm{m})$.

Figure 27. Reconstruction of a nucleus at the pachytene - diplotene transition (number 61 ). (Magnification approximately 9,000 times).

Figure 28. High magnification of recombination nodules (denoted by arrows) in longitudinal and cross sections. Figures $28 a-b$, late zygotene; Figures $28 c-d$, early pachytene; Figures $28 \mathrm{e}-\mathrm{h}$, mid pachytene. (Bar $=0.2 \mu \mathrm{m}$ ).

Figure 29. Chromatin nodules (denoted by arrows) in longitudinal and cross section. Figures 29a-b, late pachytene; Figures $29 \mathrm{c}-\mathrm{d}$, pachytene-diplotene; Figures $29 \mathrm{e}-\mathrm{f}$, early diplotene; Figures $29 \mathrm{~g}-\mathrm{h}$, mid diplotene chiasma. $($ Bar $=0.2 \mu \mathrm{m})$. 

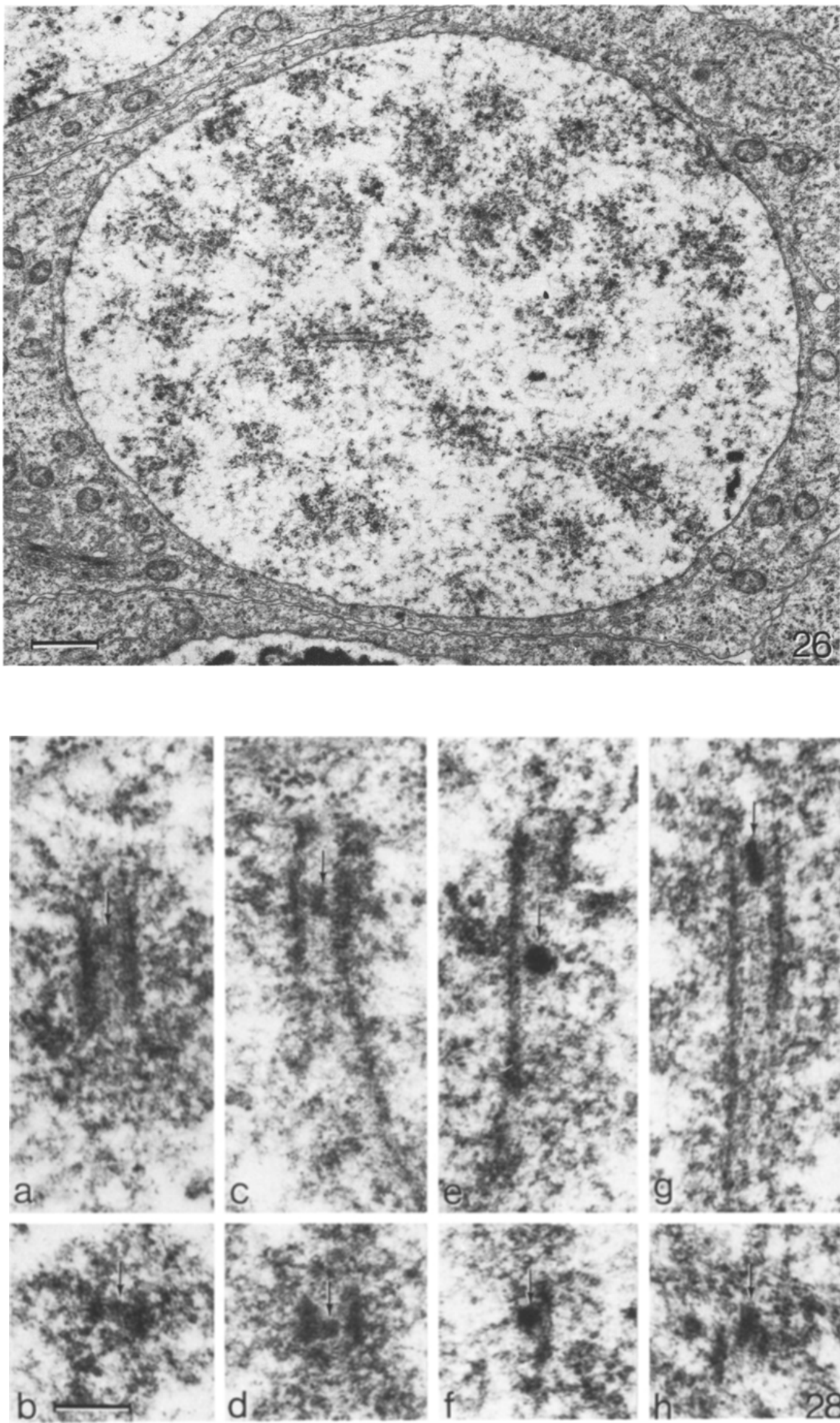

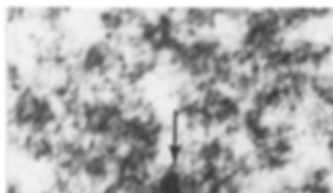
Exit?

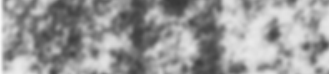

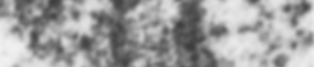
5 .

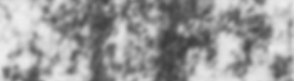

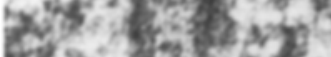

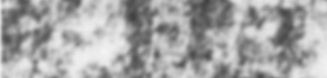

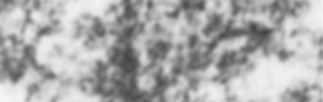

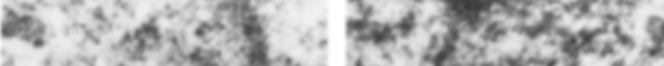

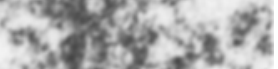

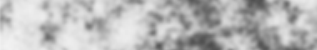

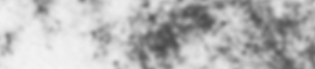
C ktestatis

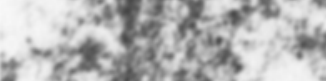

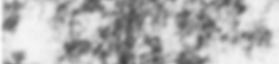
g)
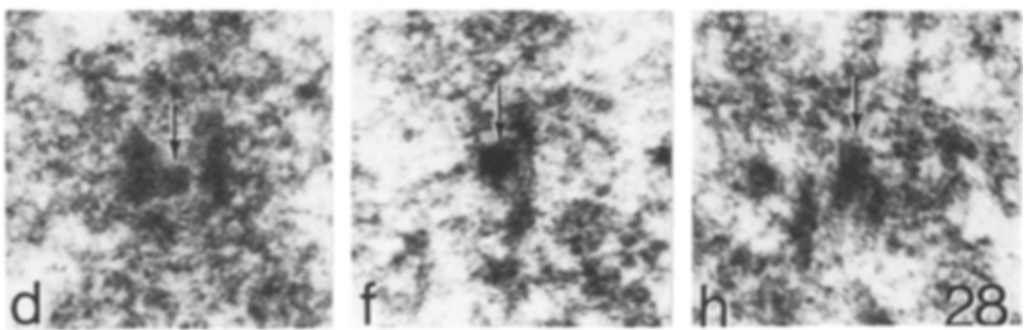


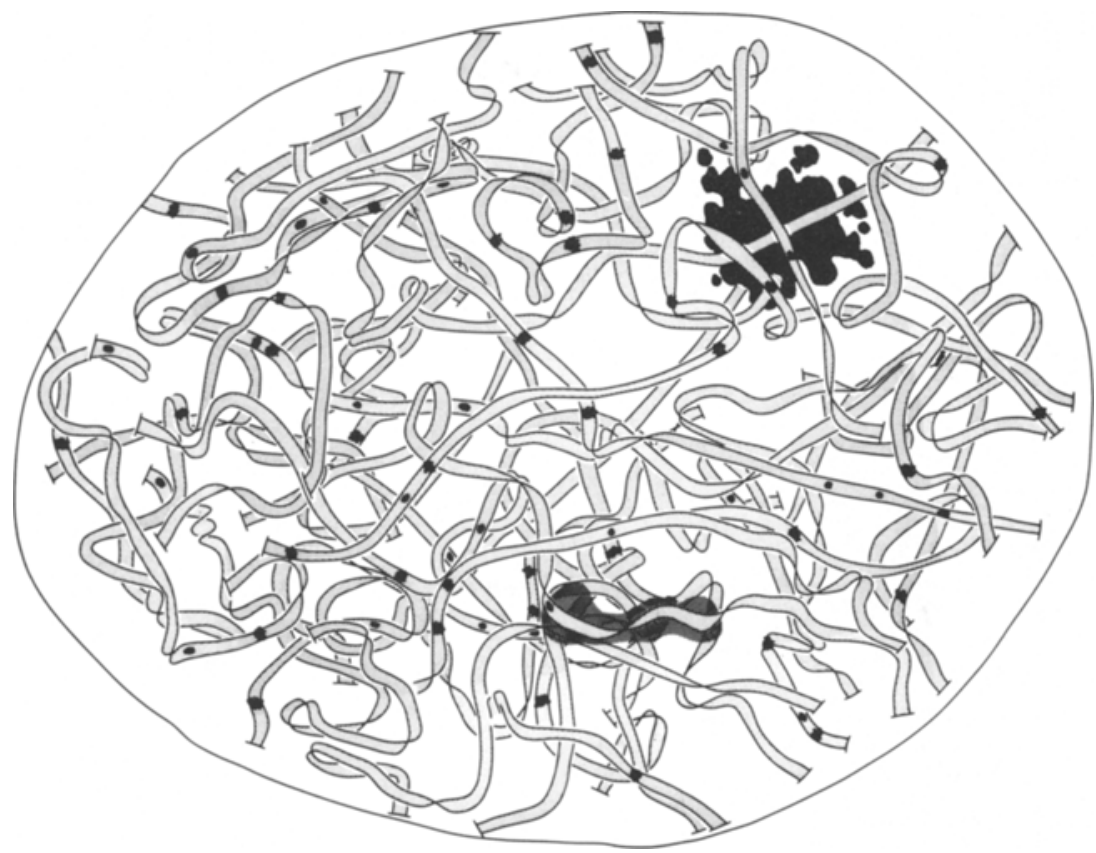

27

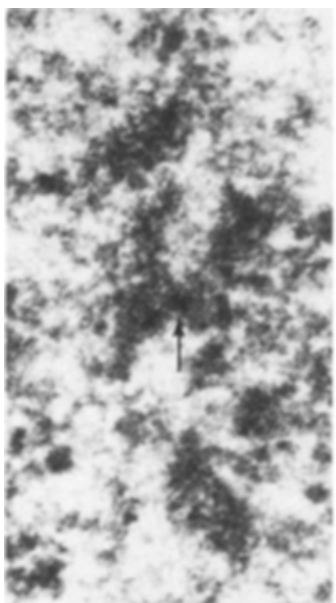

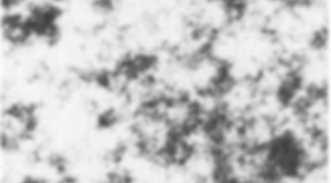
$a^{2} \cdot b^{2} y^{2}+y^{2}$

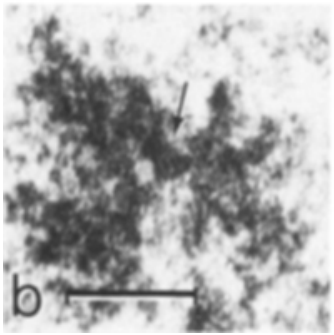

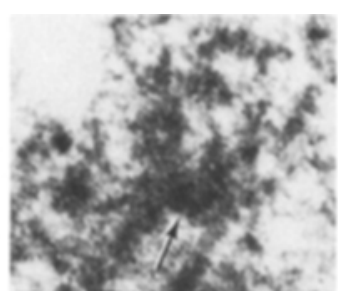

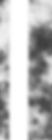




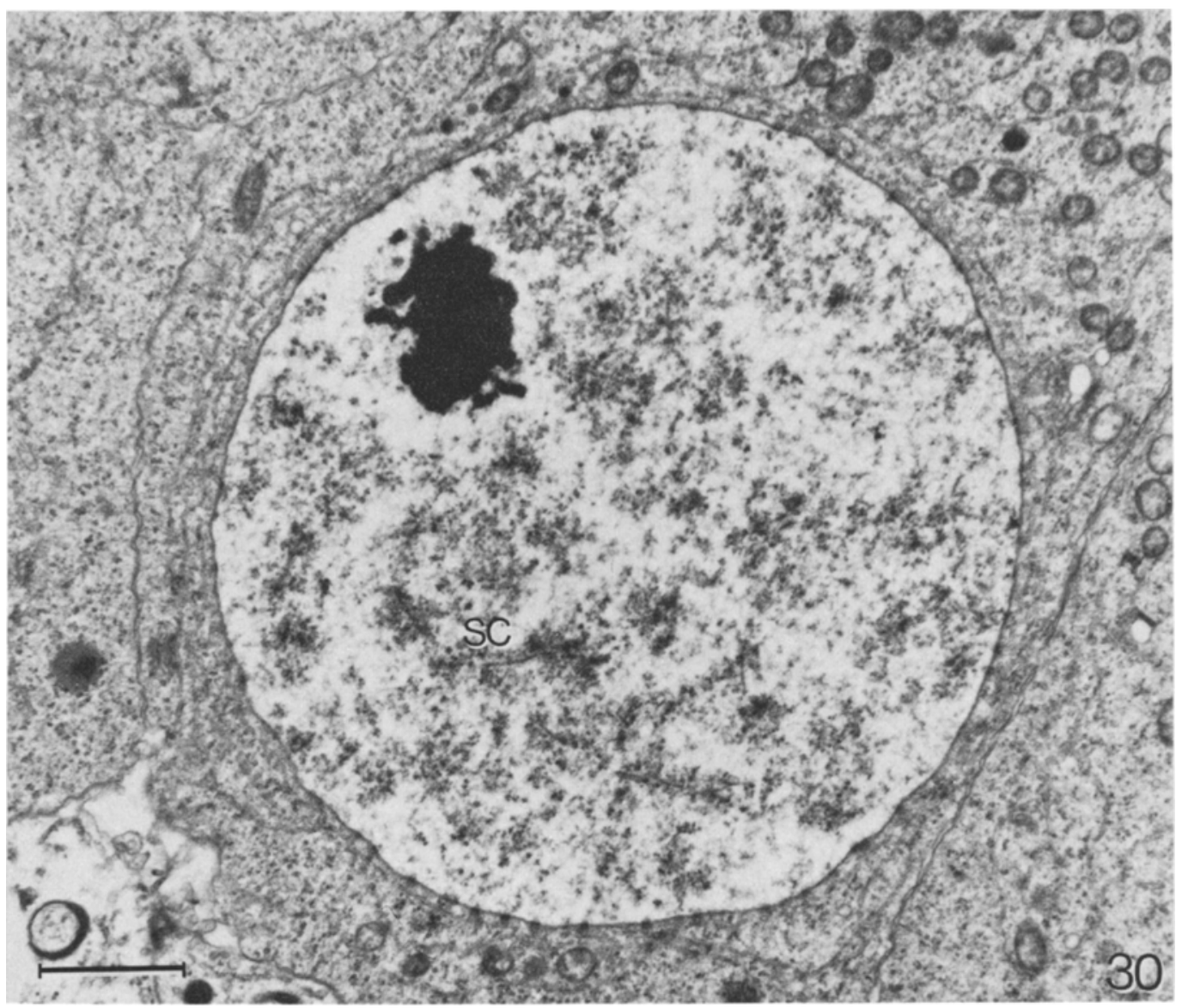

Figure 30. Survey micrograph of an early diplotene nucleus. SC, remnants of synaptonemal complexes. (Bar $=$ $2 \mu \mathrm{m})$.

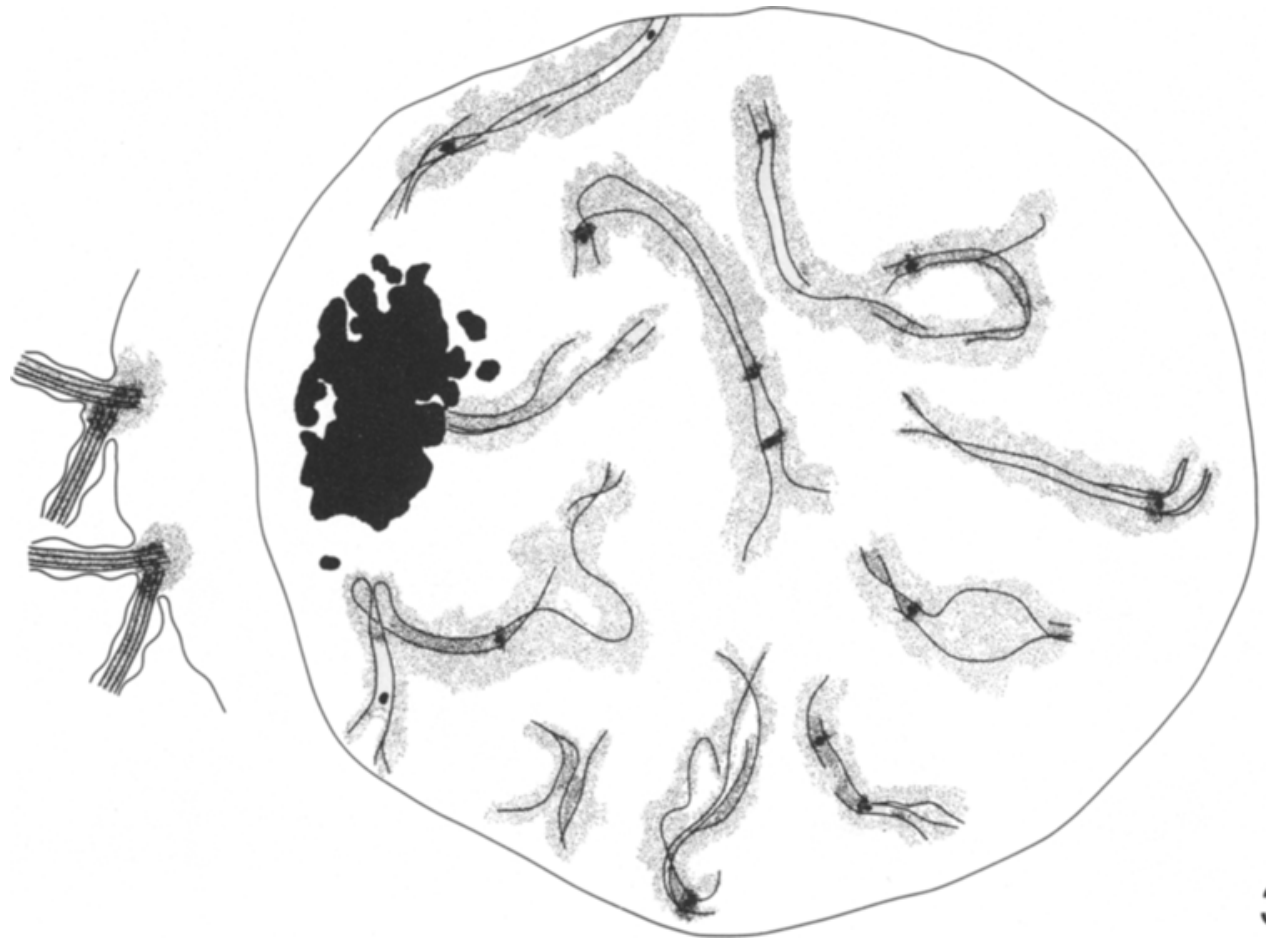

Figure 31. Partial reconstruction of an early diplotene nucleus (number 19). (Magnification approximately 9,000 times). 


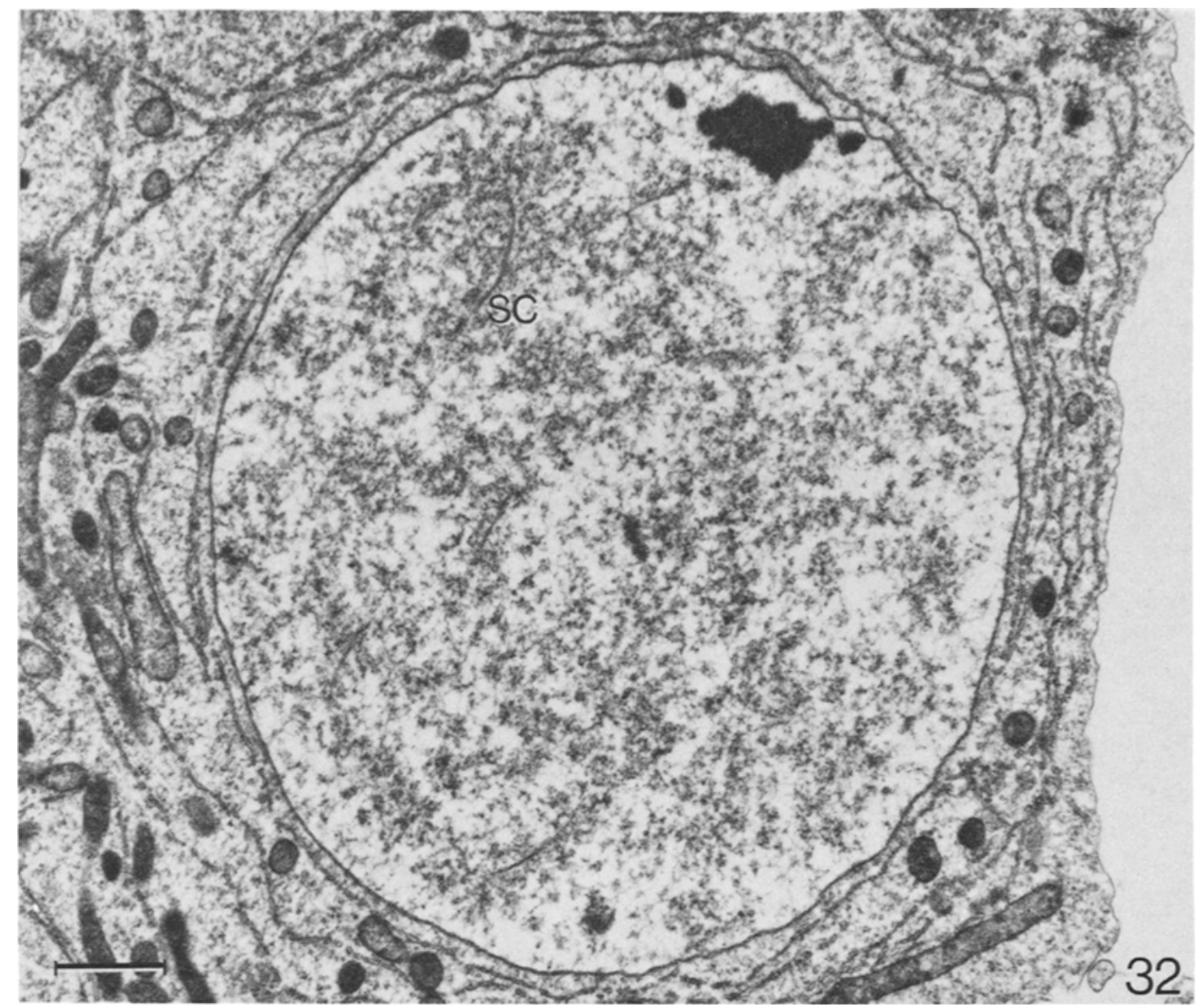

Figure 32. Survey micrograph of a mid diplotene nucleus. SC, remnants of synaptonemal complexes. $($ Bar $=$ $1.0 \mu \mathrm{m})$.

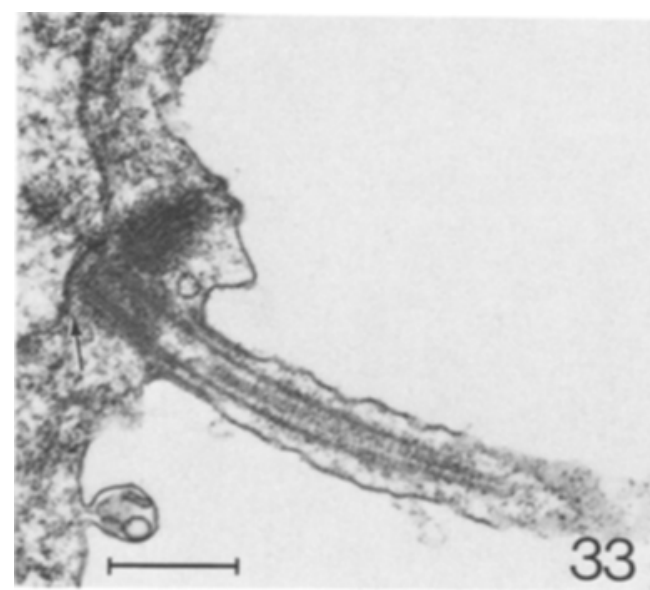

Figure 33. Centrioles of a mid diplotene nucleus. The base of the centriole is in contact with the nuclear envelope (arrow) while the flagellar part of the centriole, surrounded by the cell membrane, protrudes into the extracellular space. (Bar $=0.5 \mu \mathrm{m}$ ).

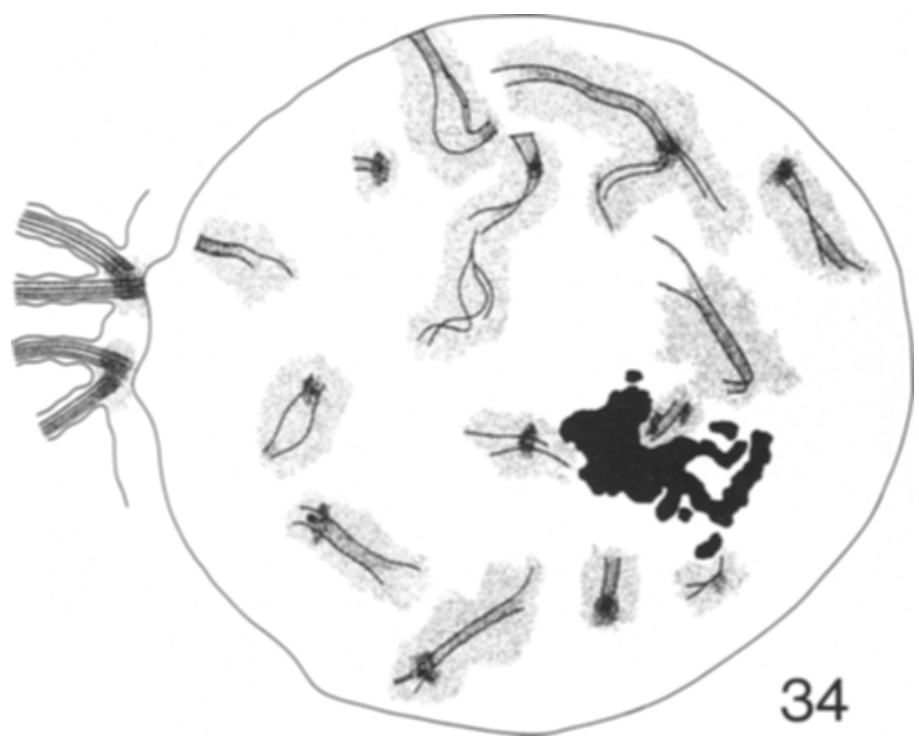

Figure 34. Partial reconstruction of a mid diplotene nucleus (number 67). (Magnification approximately 9,000 times). 


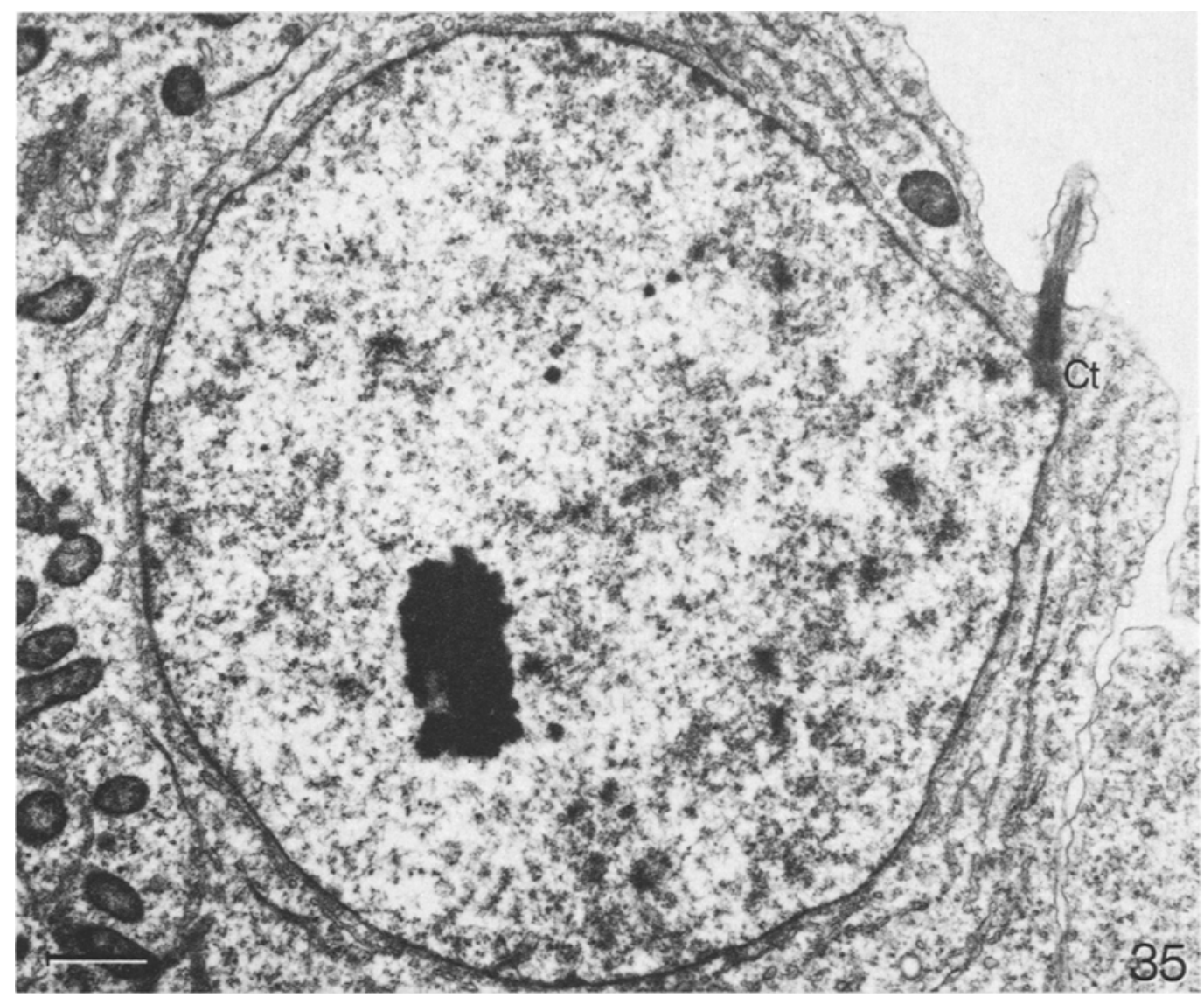

Figure 35. Survey micrograph of a late diplotene nucleus. Ct, centriole. $($ Bar $=1.0 \mu \mathrm{m})$.

is intimately associated with the chiasma. (The diagrammatic representation of the circular component of the chiasma in Figure $42 \mathrm{~b}$ is merely meant as an aid resolving the micrograph in Figure 42a and should not be considered as an exact tracing of the micrograph). The fine structure is only observable in favorable sections. In most cases, the chiasmata are identifiable as dense, ellipsoid to circular centers with diameters about $120-160 \mathrm{~nm}$ flanked by two regions of compact chromatin. The ellipsoid shape is explicable if the plane of sectioning is not coinciding with the plane determined by the circular component.

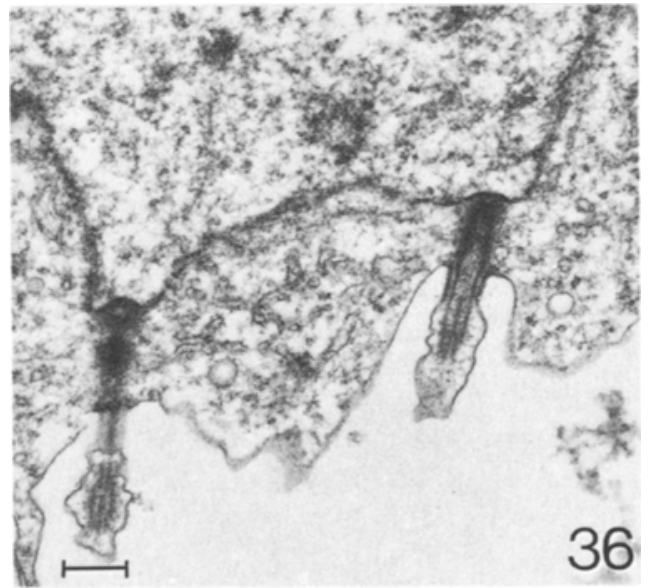

Figure 36. Centrioles at late diplotene. (Bar = $0.5 \mu \mathrm{m})$. 


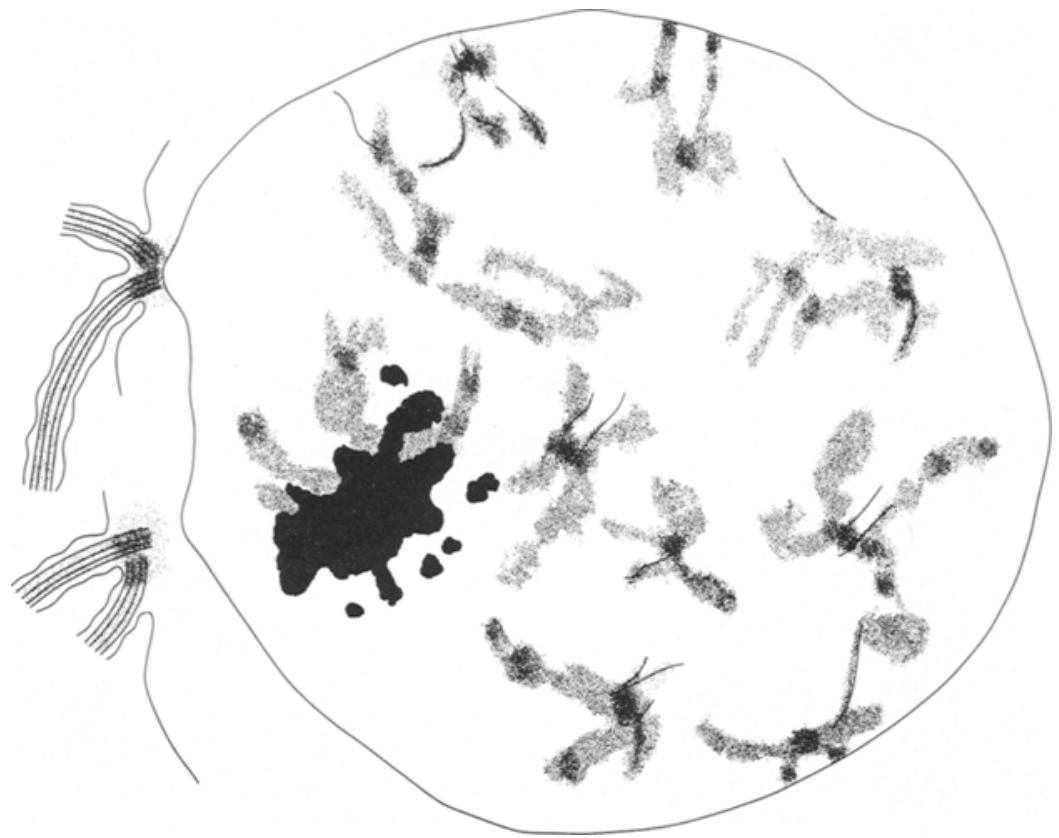

Figure 37. Partial reconstruction of a late diplotene nucleus (number 52). (Magnification approximately 9,000 times).
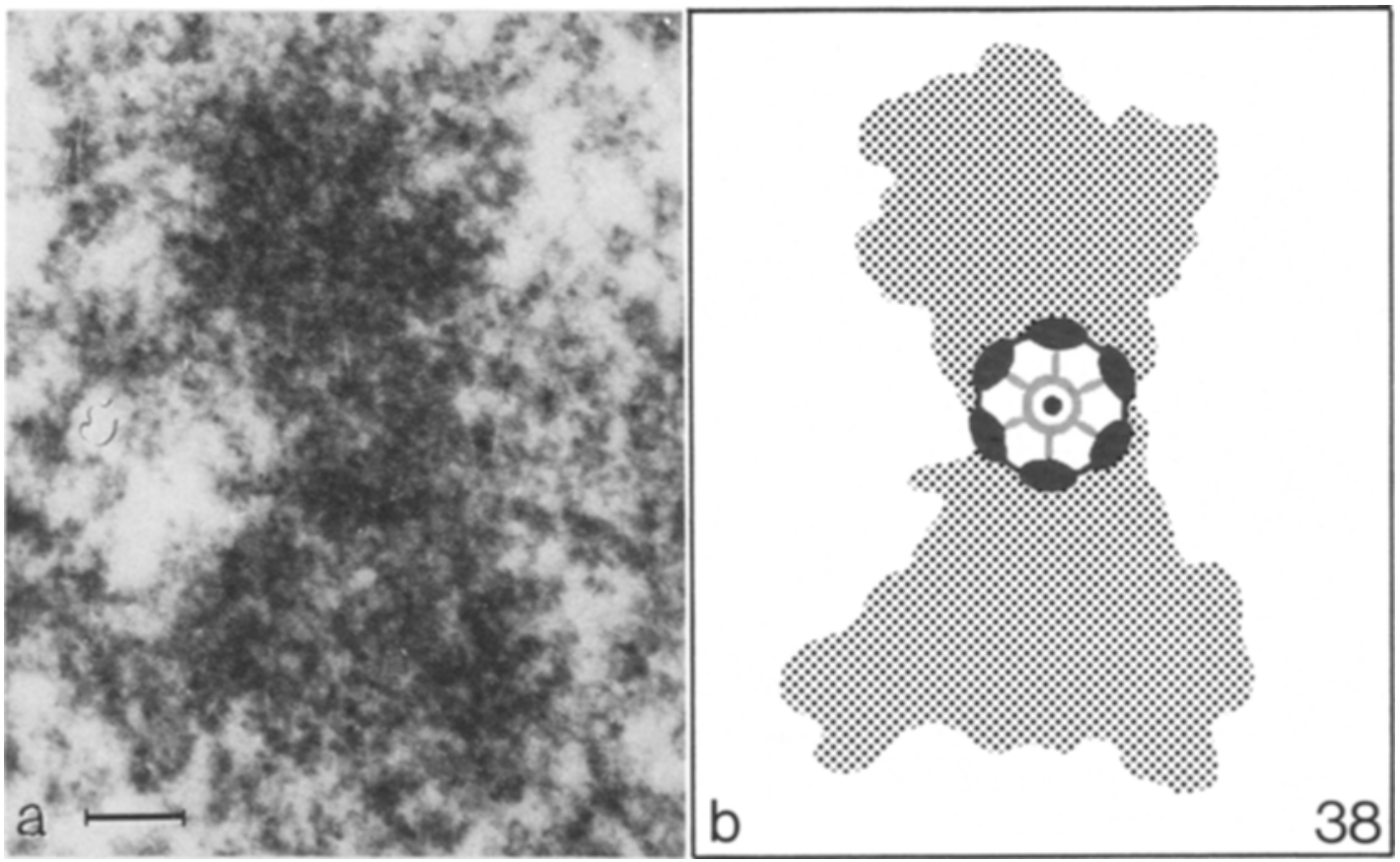

Figure 38. High magnification of a chiasma at late diplotene, Figure 38a, and a diagrammatic representation of the micrograph, Figure 38b. $($ Bar $=0.1 \mu \mathrm{m})$. 

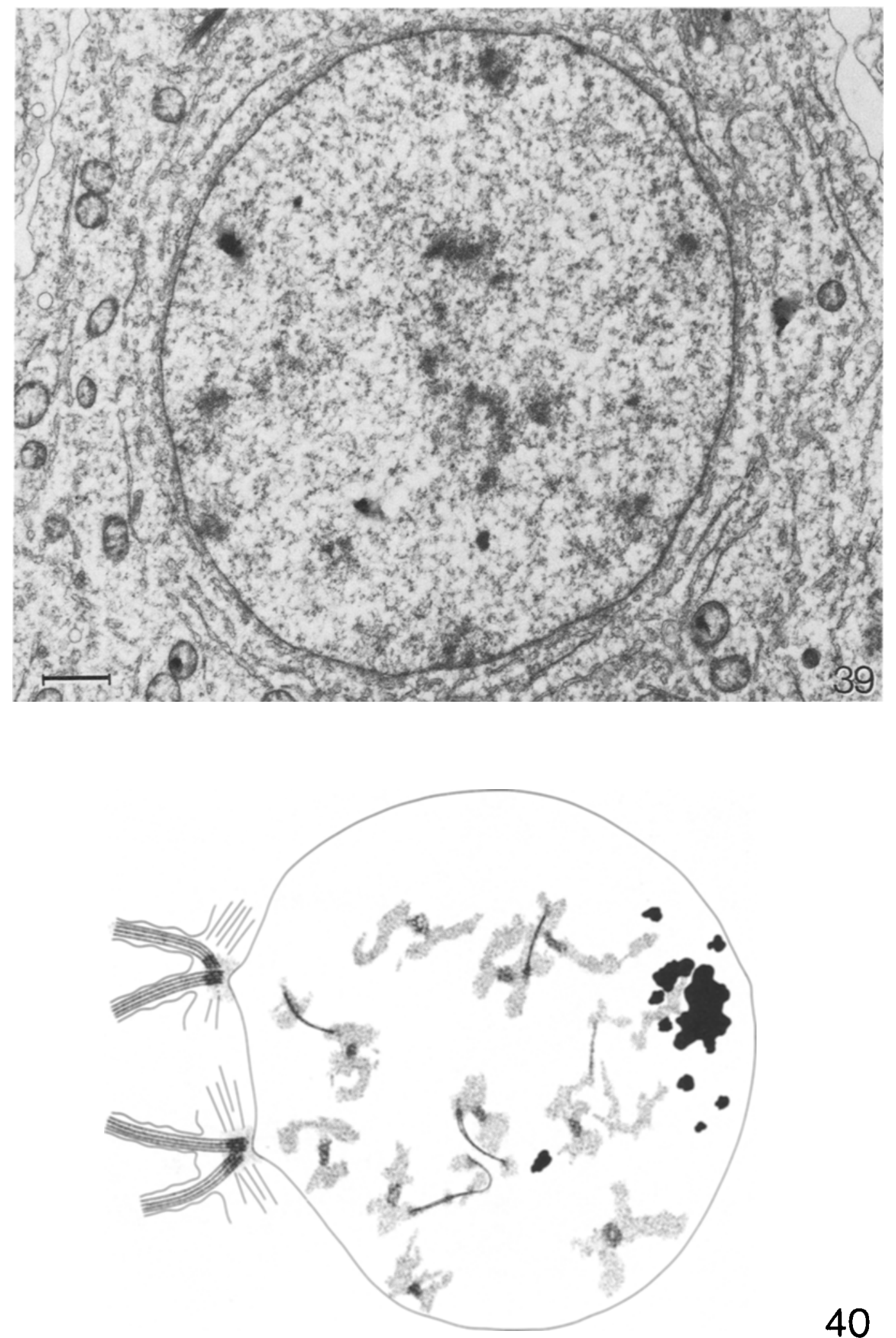
Figure 39. Survey micrograph of a nucleus at the transition from diplotene to diakinesis. (Bar $=1.0 \mu \mathrm{m})$.

Figure 40. Partial reconstruction of a nucleus (number 53) at diplotene-diakinesis. (Magnification approximately 9,000 times).

\subsection{Early diakinesis (nucleus 59)}

The centriolar base is no longer attached to the outer membrane of the nuclear envelope but at each of the former attachment sites an invagination of the nuclear envelope has evolved (Figure 44). Numerous nucleolar fragments are at this stage dispersed over a large part of the nucleus.

The condensation of the chromatin, initiated at the transition from diplotene to diakinesis progresses during early diakinesis (Figure 43) making reconstructions of longer bivalent segments feasible (Figure 44) although reconstruction of the entire chromosome complement by the chromatin contour is still impossible. Partial reconstruction of bivalents reveals typical chiasmata flanked by condensed chromatin (Figure $45 \mathrm{~b}$ ). On both sides of the chiasma two segments of condensed chromatin can be followed for a short distance (Figure 44).

\subsection{Mid diakinesis (nuclei $58,60,61 ; 58$ partially reconstructed)}

Nucleolar degradation is by mid diakinesis almost complete with only a few fragments remaining (Figures 46 and 47). The separation of the two pairs of centrioles which is initiated at this stage, coincides with the separation also of the nuclear invaginations formed during early diakinesis.

Chromatin condensation has now reached a level which permits the reconstruction of all 28 bivalents by their chromatin. Although the two homologues generally are close together, it is often possible to distinguish and reconstruct the individual homologues of each bivalent. The eight consecutive sections in Figure 48 show a part of a bivalent with two chiasmata (arrows in Figures $48 \mathrm{c}$ and $\mathrm{f}$ ). As seen in the micrographs, the chiasmata are identifiable by a chromatin bridge connecting the two homologues and has an almost circular (Figure 48c) or more irregular (Figure 48f) component in the middle. In the higher magnification in Figure 49, it is possible to distinguish remnants of the substructures characteristic of the circular component of the chiasma at earlier stages as outlined in the accompanying schematic drawing (Figure 49b).

\subsection{Late diakinesis (nuclei $29-32 ; ; 0$ and 32 incomplete)}

Separation of the two pairs of centrioles proceeds during late diakinesis and is, as in the previous stage, accompanied by a corresponding separation of the two nuclear invaginations (Figure 51). Several microtubules radiate out from the proximal part of the centrioles and in the cytoplasm parallel sheets of membranes appear

The bivalents are at this stage heavily condensed and resolution of the two homologues of each bivalent is in most cases impossible (Figures 50 and 51). Some bivalents possess one or two of the circular components of the chiasma but in most bivalents only remnants are present and a few bivalents appear to be devoid of the circular structures. Although the exact number could not be determined it was evident that they were less frequent than at mid diakinesis and earlier stages (see section 3.19.2.).

\subsection{Metaphase I (cells 33-36, 75)}

The two pairs of centrioles with their flagellae have at metaphase I moved to opposite poles and at each pole a reticulum of vesicles has evolved. Between the poles, a number of membrane compartments has formed, each including one bivalent (Figures 52 and 53).

In the four cells sectioned parallel to the spindle it is possible in each of the 28 bivalents to distinguish four separate cup shaped indentations of the chromatin, two-by-two facing opposite spindle poles (denoted by arrows in Figures $55 \mathrm{e}$ and $f$ and shown in the semidiagrammatic reconstruction in Figure 54). Within these regions, a mass of low electron density is 

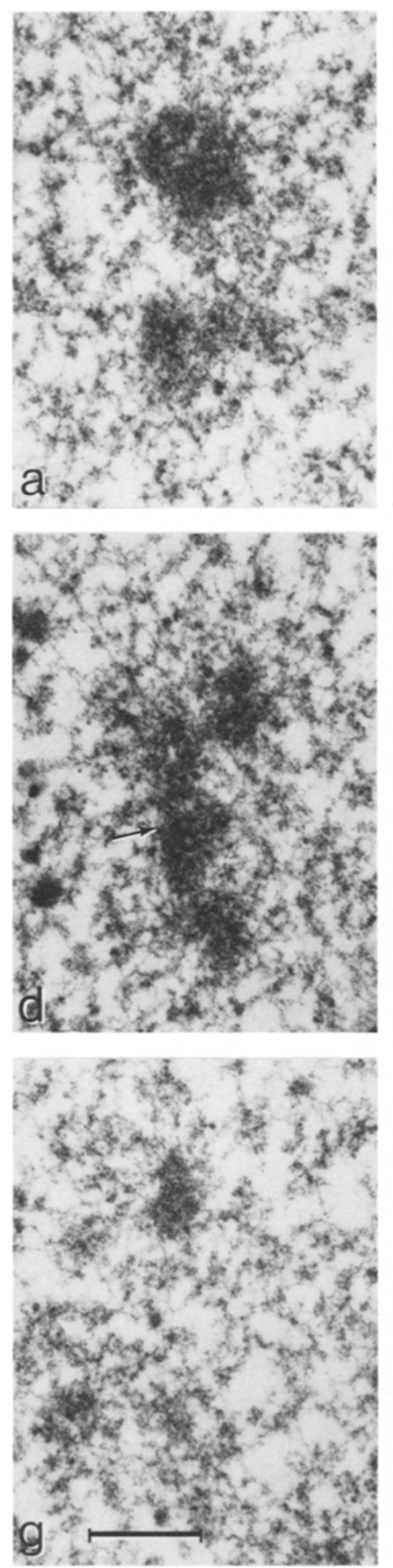
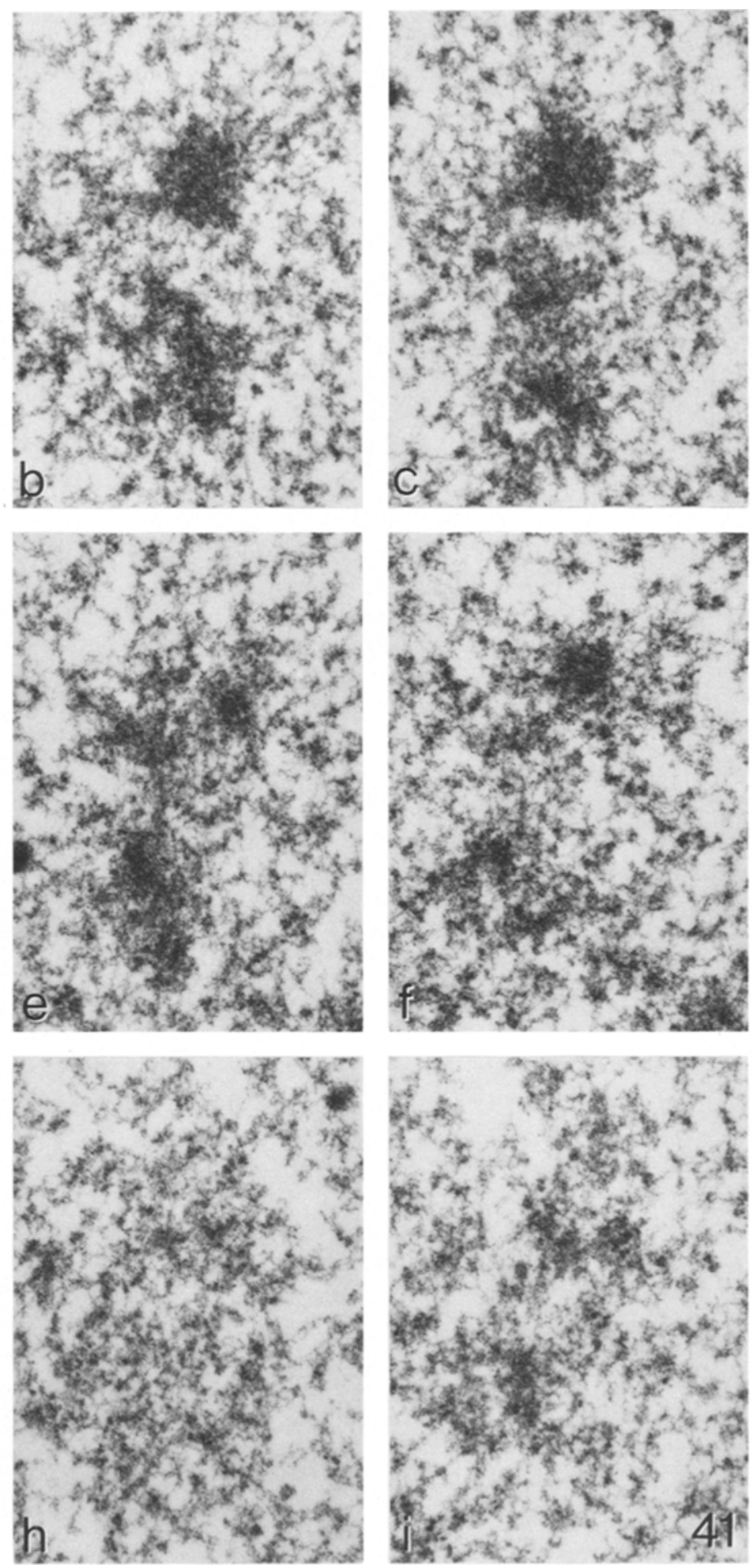

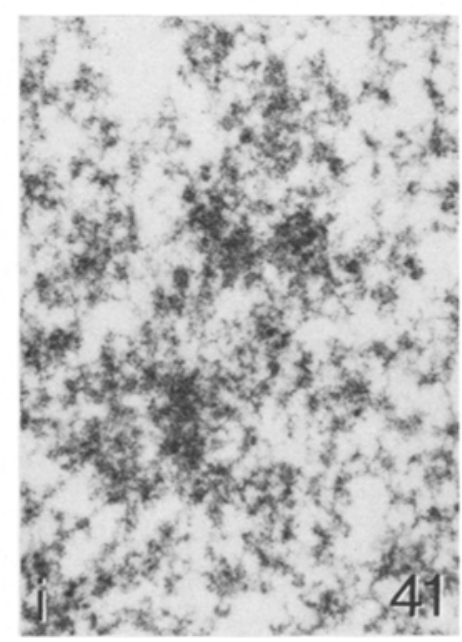

Figure 41. Nine consecutive sections through a chiasma at the diplotene - diakinesis transition. Note the circular component of the chiasma (denoted by arrow in Figure $41 \mathrm{~d}$ ). (Bar $=0.5 \mu \mathrm{m}$ ). 


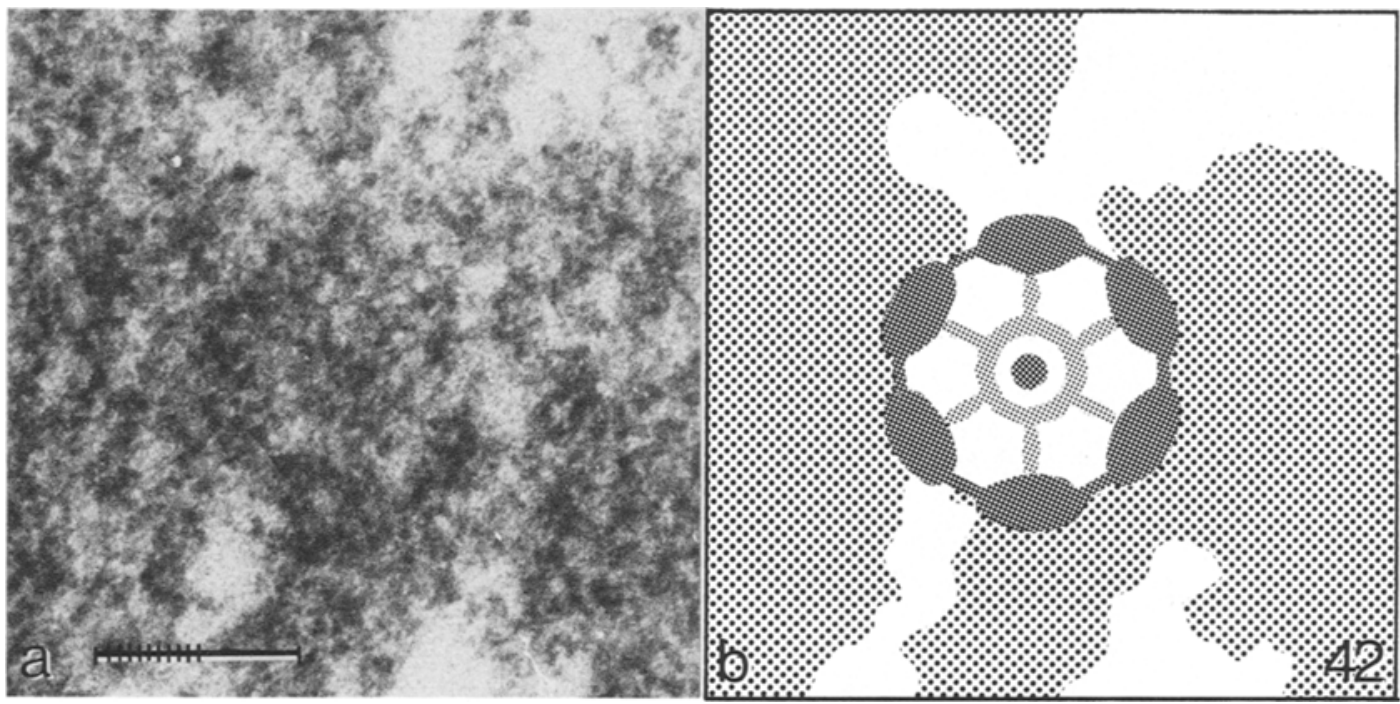

Figure 42. High magnification of the circular component of the chiasma at the diplotene - diakinesis transition. A diagrammatic interpretation of the micrograph is shown in Figure 42b. $(\mathrm{Bar}=0.1 \mu \mathrm{m})$.

present, and frequently, a few microtubules extend from this material giving the regions the appearance of localized centromeres. Very few microtubules are present in all five cells despite the fact that all bivalents have congressed into a well-defined metaphase plate.

Chromatin condensation has at metaphase I reached its maximum and the two homologues of each bivalent cannot be distinguished. As shown in the eleven consecutive sections through a bivalent in Figure 55 and the diagrammatic representation of the micrographs in Figure $55 \mathrm{~m}$, chromatin condensation is less pronounced in the center of the bivalents, the border between the central and the dense outer part being welldefined (see Figures 55e and $f$ which are sections approximately through the center of the bivalent). In a few bivalents, the circular components of chiasmata characteristic of earlier stages can be identified at the presumed border between the homologues (Figure 56).

\subsection{Number and distribution of nodules and chiasmata}

\subsubsection{Total number of nodules per nucleus}

The total number of nodules per nucleus at late zygotene, early pachytene, mid pachytene, late pachytene and the pachytene - diplotene transition are given in Tables I and V-VIII and are summarized in Table IX.

At late zygotene, the mean number of nodules per nucleus is $91 \pm 14$ ranging from 72 to 106 . On the assumption that yet unpaired regions will have the same probability for receiving nodules, the expected number is $103 \pm 21$ with a range from 82 to 141 . At this stage, as well as at succeeding stages, there is no correlation between the total complement length and the total number of nodules per nucleus.

The total number of nodules decreases at early pachytene to a mean of $61 \pm 14$ ranging from 36 to 80 (Table V). This trend continues at mid pachytene where the mean number of nodules per nucleus is $55 \pm 8$. The number of nodules again increases during late pachytene and pachytene-diplotene where the mean numbers are 70 \pm 7 and $75 \pm 15$, respectively, ranging from 58 to 78 at late pachytene and from 61 to 91 at pachytene-diplotene (Tables VII and VIII).

From the data summarized in Table IX, it can be seen that the number of nodules decreases by $47 \%$ from late zygotene to mid pachytene. Thereafter, the number of nodules again increases to a value equal to $73 \%$ of the number expected upon completion of pairing at late 


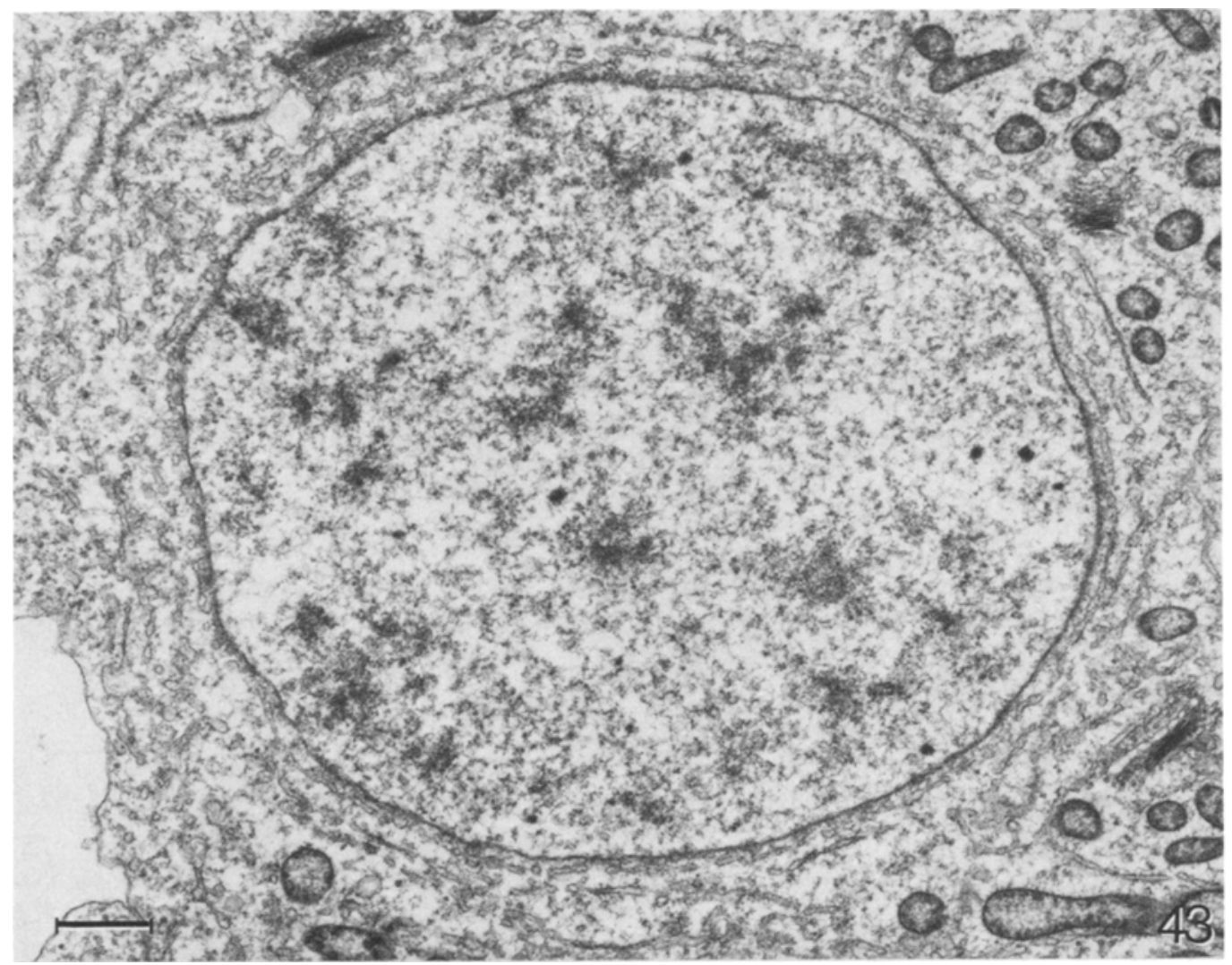

Figure 43. Survey micrograph of a nucleus at early diakinesis. (Bar $=1.0 \mu \mathrm{m})$.

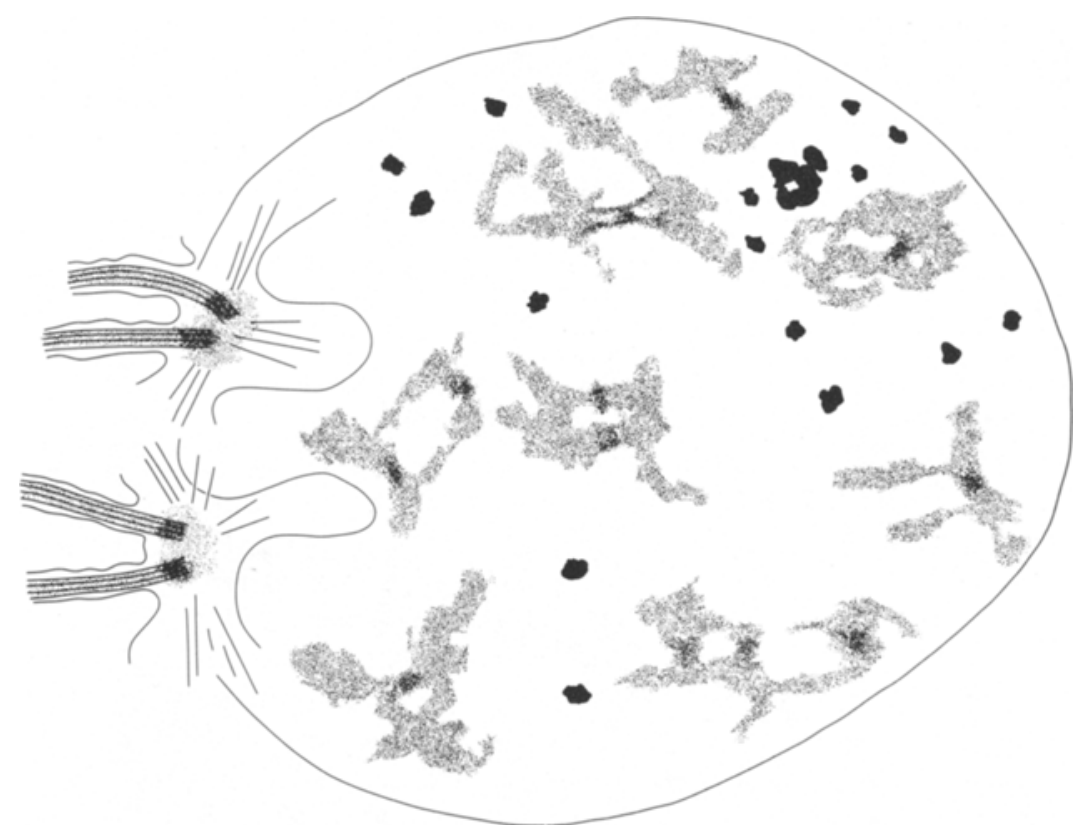

Figure 44. Partial reconstruction of an early diakinesis nucleus (number 59). (Magnification approximately 9,000 times). 

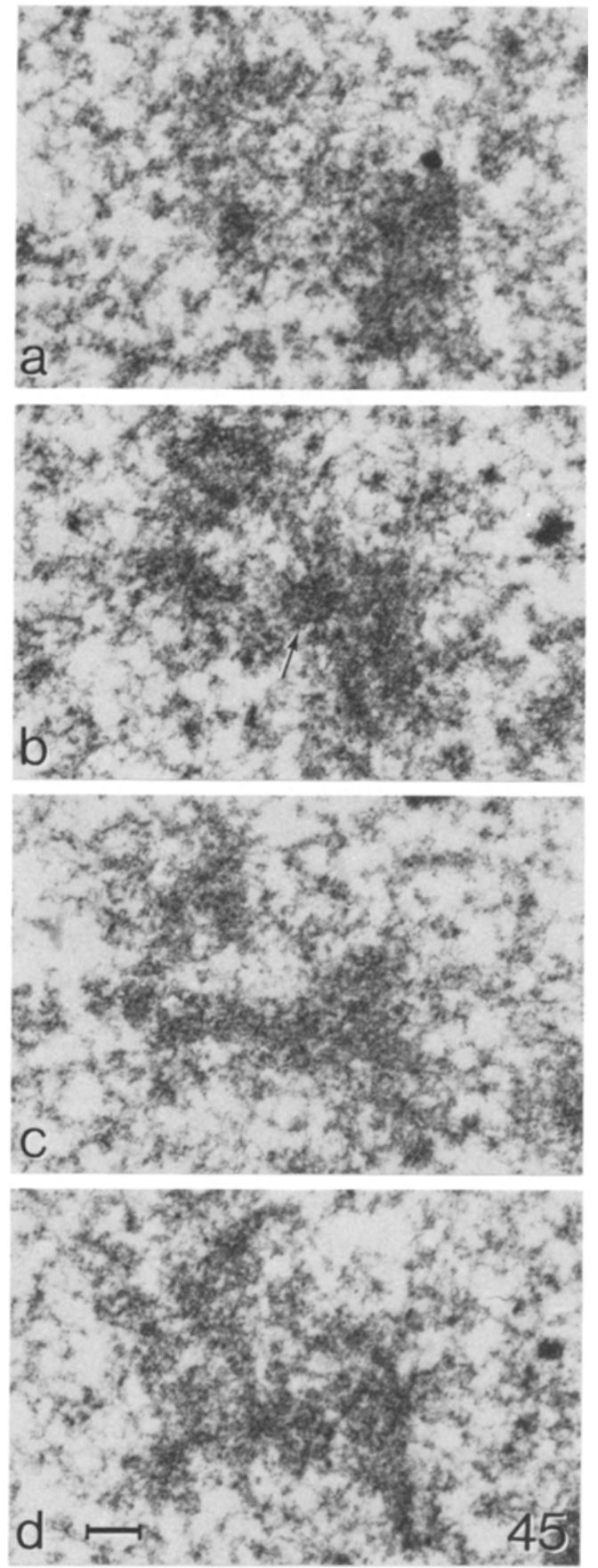

Figure 45. Four consecutive sections through a chiasma at early diakinesis. Note in Figure $45 \mathrm{~b}$ the circular component of the chiasma (denoted by arrow). (Cf. Figures 38 and 42). (Bar $=0.2 \mu \mathrm{m}$ ). zygotene. The changes in the total number of nodules are accompanied by a change also in the number of recombination nodules relative to the number of chromatin nodules: The ratio of chromatin nodules to recombination nodules increases from 0 at late zygotene through 0.03 at early pachytene, 0.49 at mid pachytene, 1.7 at late pachytene to 3.7 at the pachytene - diplotene transition. This is consistent with the proposal that the chromatin nodules evolve from recombination nodules (see section 3.10.).

\subsubsection{Total number of chiasmata per nucleus}

In the following, the term chiasma refers to regions, which at mid diplotene are characterized by the presence of differentially condensed chromatin filling and partly surrounding short synaptonemal complex fragments, and which in the central region of the complex often contain a dense core reminiscent of a recombination nodule. From late diplotene to metaphase I chiasmata are identified by their circular component as described in sections 3.13.- 3.18.

As illustrated in Table $\mathrm{X}$, the number of chromatin nodules at early diplotene is very similar to the number of chiasmata at mid diplotene. From mid diplotene to mid diakinesis, the number of chiasmata remains fairly constant with a mean value for all stages of 58 per nucleus. By late diakinesis, the progression of chromatin condensation and the gradual elimination of the circular component of the chiasmata render a realiable identification of chiasmata difficult except in cases where the circular components is still present. The reduction of the number of circular components possibly marks the initiation of chiasma terminalization. If this is the case, chiasma terminalization is almost complete by metaphase I as judged by the very low number of circular components.

\subsubsection{Distribution of nodules among bivalents}

In order to analyse the distribution of nodules among the 28 bivalents at the different stages of early meiotic prophase, the number of bivalents with $0,1,2, \ldots,>7$ nodules was determined for each stage and compared with the numbers one would expect if the nodules were distributed randomly among the bivalents. The expected, 


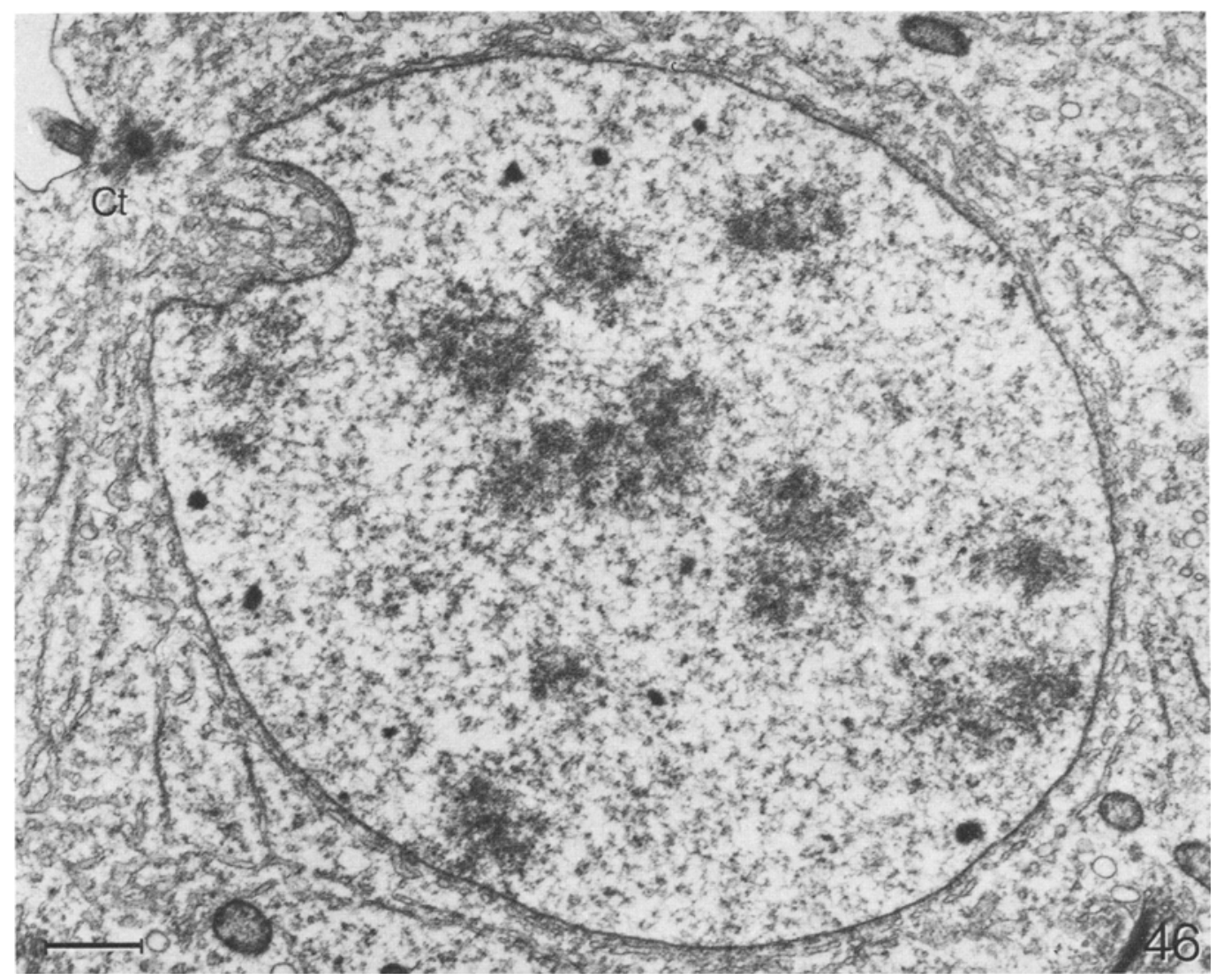

Figure 46. Survey micrograph of a mid diakinesis nucleus. $\mathrm{Ct}$, centrioles. $(\mathrm{Bar}=1.0 \mu \mathrm{m})$.

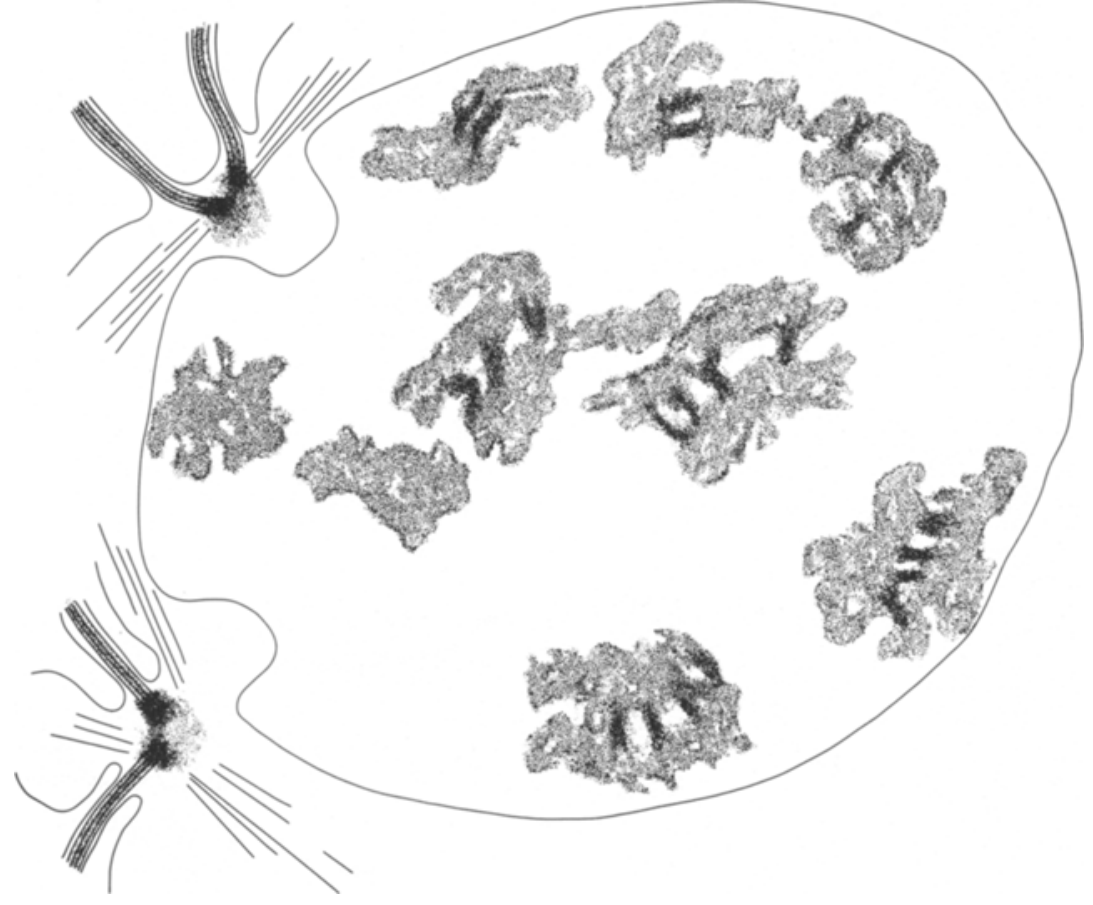

Figure 47. Partial reconstruction of a mid diakinesis nucleus (number 6la). Note the two univalents. (Magnification approximately 9,000 times). 

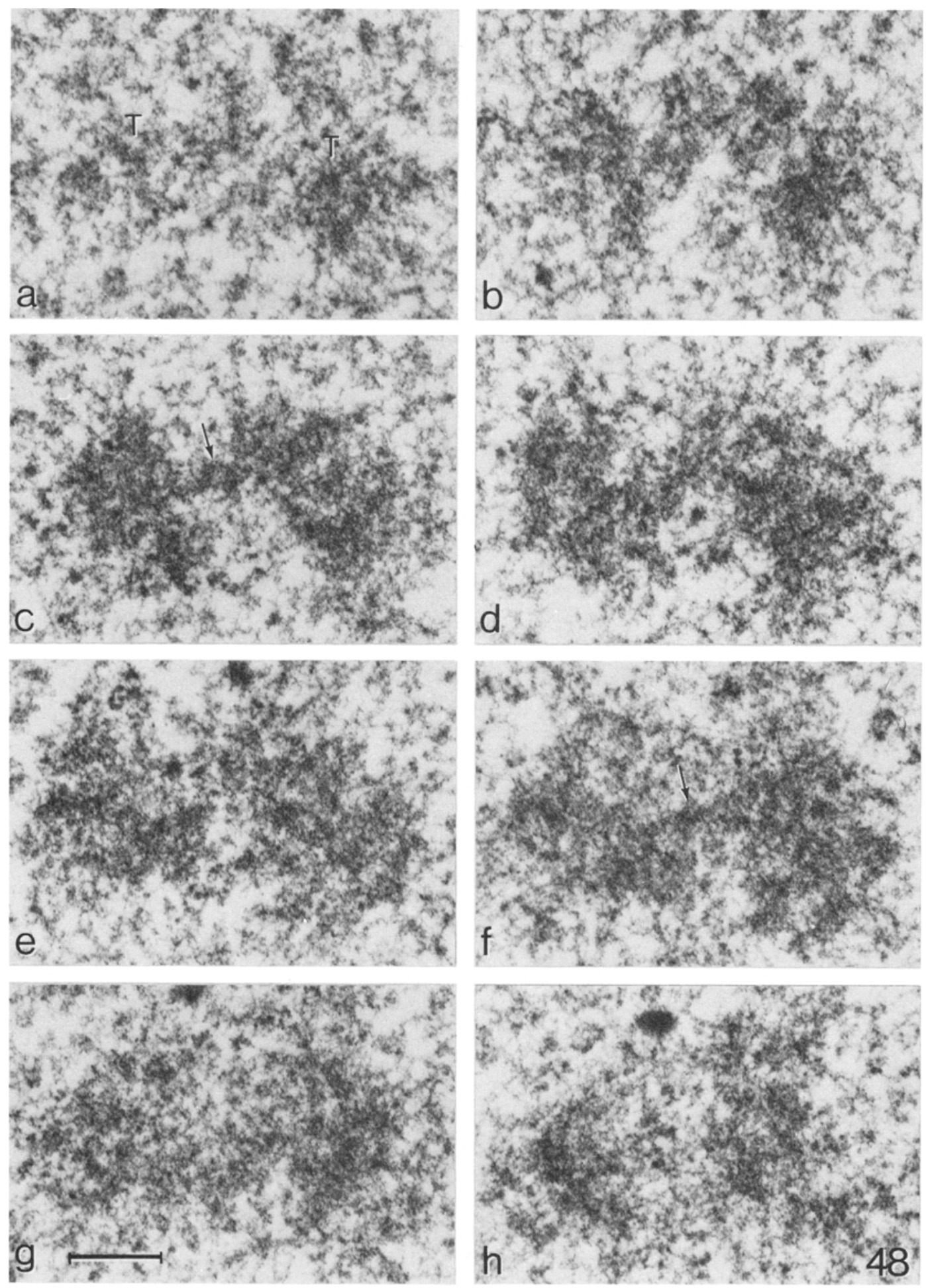

Figure 48. Eight consecutive sections through one half of a mid diakinesis bivalent. Two chiasmata (denoted by arrows) are included in the series one in Figure $48 \mathrm{c}$ and the second in Figure $48 \mathrm{f}$. T, telomere. $(B a r=0.5 \mu \mathrm{m})$. 


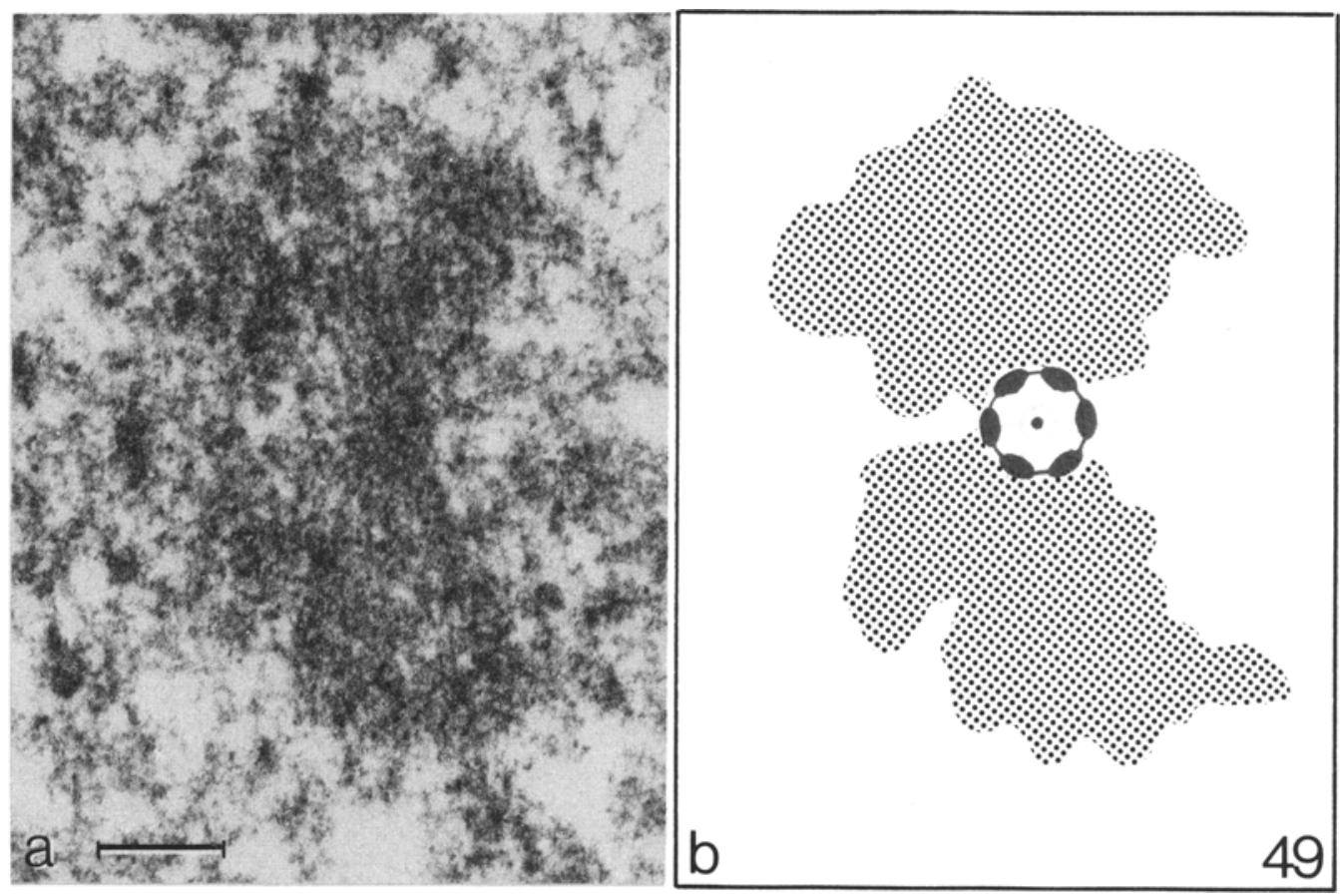

Figure 49. High magnification of a chiasma at mid diakinesis. A diagrammatic interpretation of the micrograph is given in Figure $49 \mathrm{~b}$. Note the change in ultrastructure of the circular component of the chiasma. (Cf. Figures 38 and 42 ). (Bar $=0.2 \mu \mathrm{m}$ ).

theoretical values were constructed by a computer simulation experiment in the following way: The absolute lengths of the individual bivalents were fed into a Honeywell Bull H6000 computer. The computer was programmed to position the nodules at random along and among the bivalents so that all segments of all bivalents had the same probability of receiving a nodule, i.e., the mean number of nodules per bivalent will be directly proportional to its length. The nodules were positioned one at a time and in those cases where the nodule position generated by the computer was closer than $80 \mathrm{~nm}$ to a previously positioned nodule or closer than $40 \mathrm{~nm}$ to a telomere, a new position was generated. The program was run a total of 8000 times for each stage, the number of runs for a given nucleus corresponding to 8000 divided by the number of nuclei reconstructed for that stage. In this way, the frequencies of bivalents with $0,1,2, \ldots \ldots$, $>7$ nodules and their standard deviations were determined together with all neighbour nodule and nodule-telomere distances (see section 3.19.5.). A comparison between the observed and expected frequencies in the 9 classes (Table $\mathrm{XI})$ permits the following conclusions:

1) A late zygotene, the observed and expected distributions are virtually identical with similar standard deviations, thus demonstrating a perfectly random distribution of recombination nodules among the bivalents of a nucleus.

Figure 50. Survey micrograph of a late diakinesis nucleus. LC, remnant of lateral component. (Bar $=1.0 \mu \mathrm{m})$.

Figure 51. Partial reconstruction of a late diakinesis nucleus (number 29). (Magnification approximately 9,000 times). 

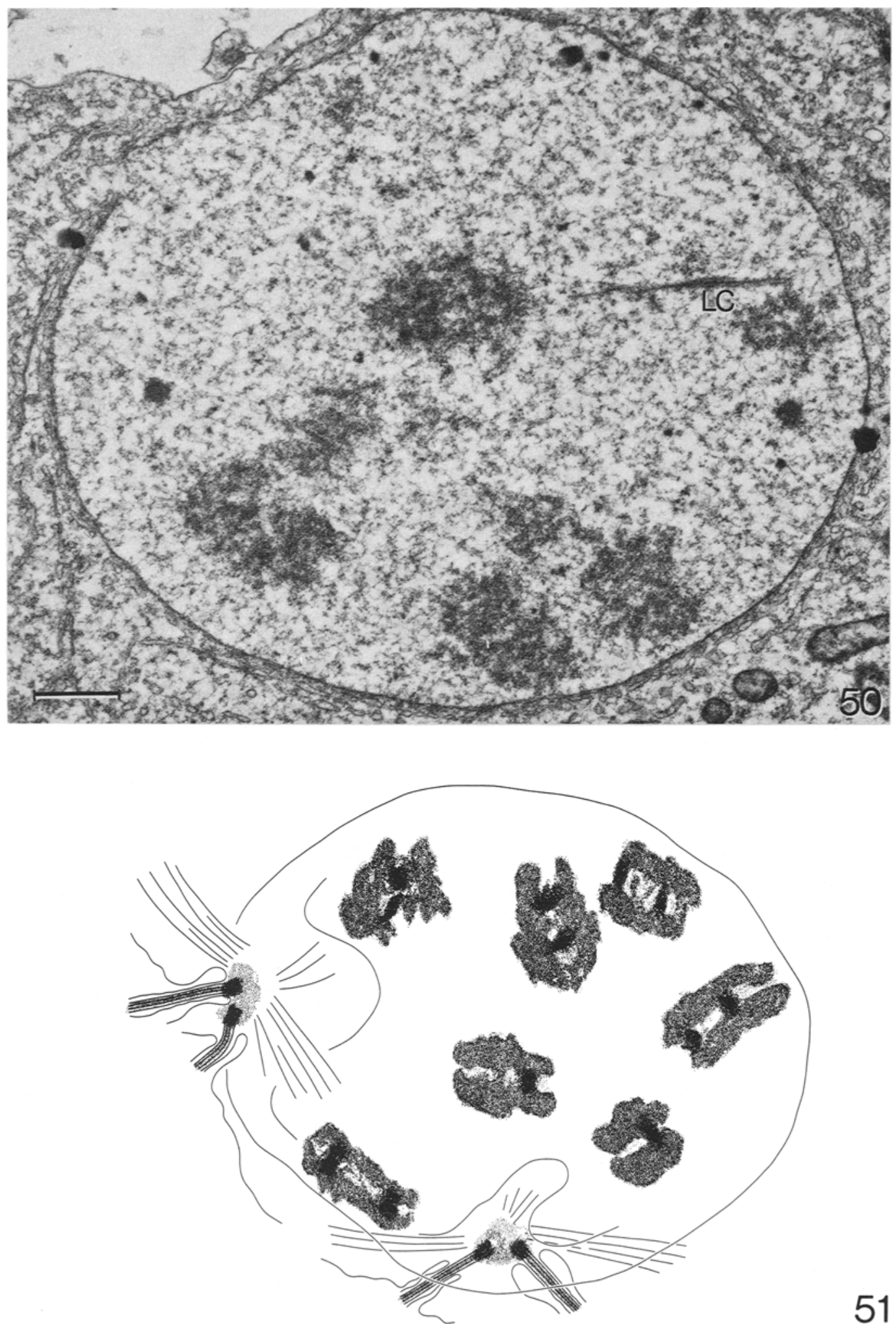

51 


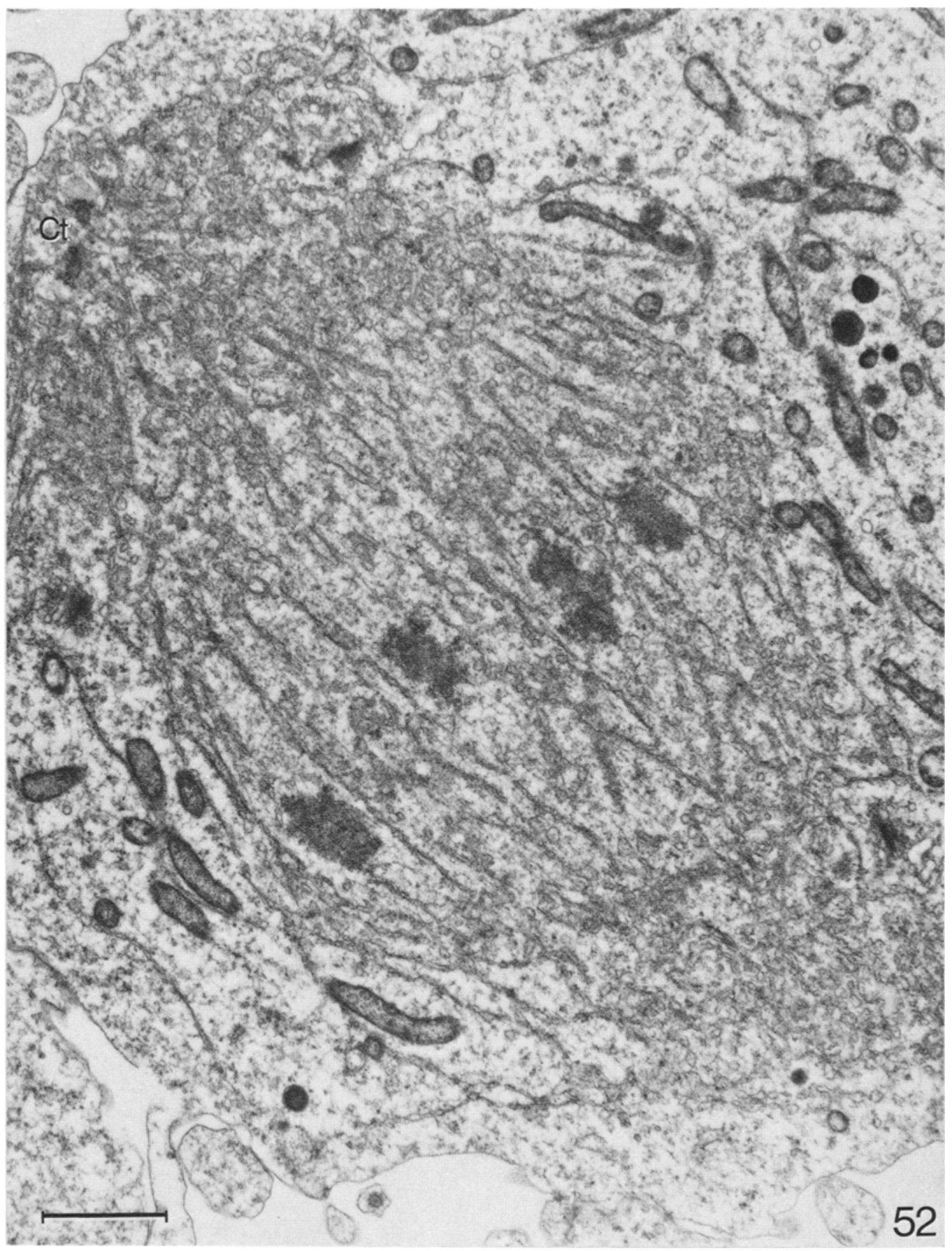

Figure 52. Survey micrograph of a metaphase I cell. Ct, centrioles. (Bar $=2.0 \mu \mathrm{m}$ ). 


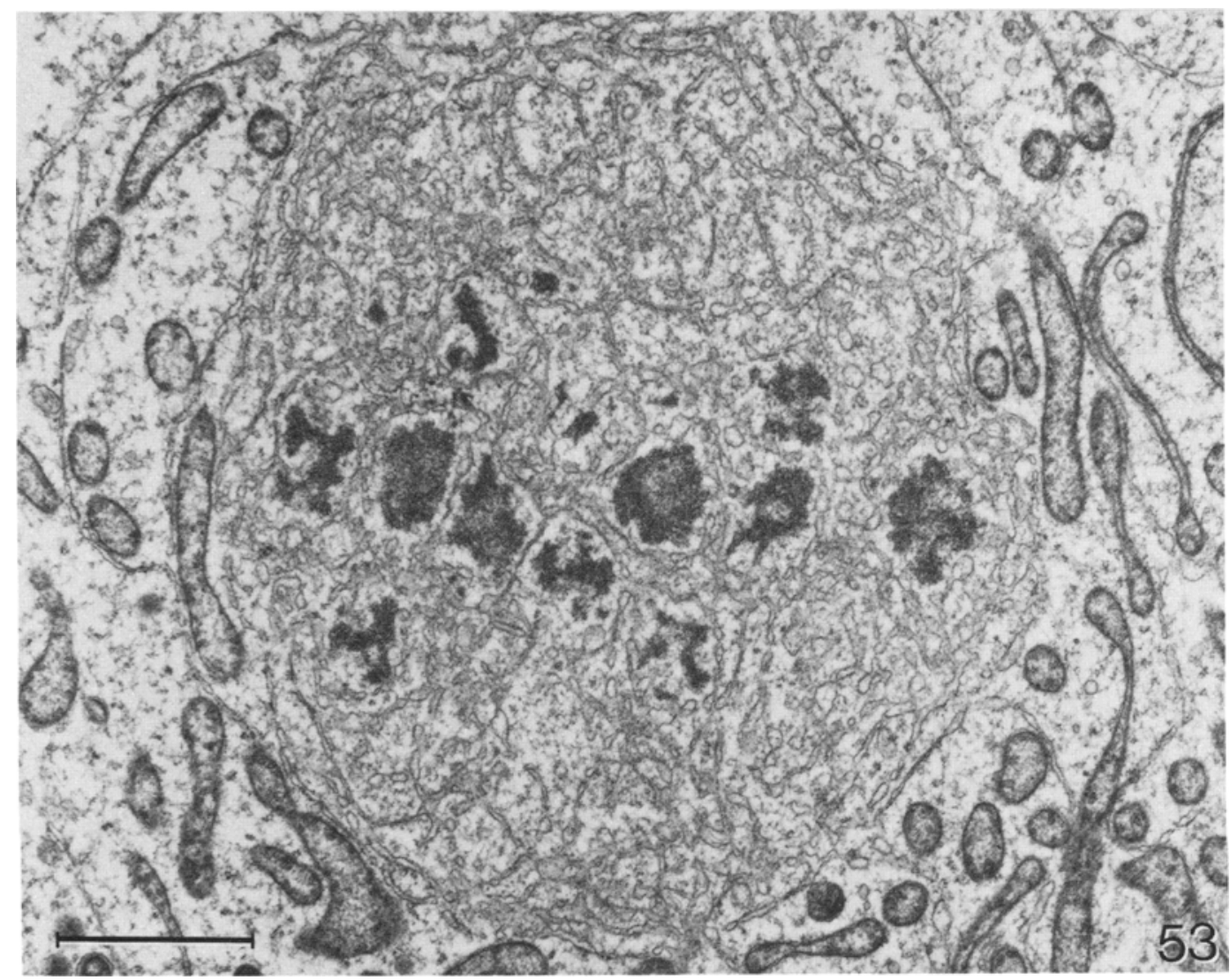

Figure 53. Survey micrograph of a metaphase I cell cut almost parallel to the equatorial plane. (Bar $=2.0 \mu \mathrm{m}$ ).

2) At early pachytene, the observed and expected frequencies are very similar, the major difference being in the frequencies of bivalents without nodules of which $13 \%$ were expected but only $8 \%$ observed. 3) At mid pachytene, the observed and expected frequencies exhibit marked differences: only $2 \%$ of the bivalents were without nodules against an expectation of $15 \%$. Furthermore, the group of bivalents with one or two nodules constitute $70 \%$ of all bivalents while only $54 \%$ were expected.

4) At late pachytene, the same pattern is observed, bivalents without nodules being much less frequent than expected whereas the opposite is the case for bivalents with 2 and 3 nodules.
Table XI also includes data for nuclei at the pachytene - diplotene transition showing the same general pattern as for mid and late pachytene. The low number of nuclei investigated together with the difficulties encountered in their reconstruction does, however, limit the reliability of the observed frequencies. A total of 47 bivalents was found without nodules (Tables I c, V-VIII) distributed among the size classes in the following way: bivalents 1-7: 7 cases; bivalents 8-14: 8 cases; bivalents 15-22: 15 cases; bivalents 23-28: 17 cases. This demonstrates that although bivalents devoid of nodules are found in all size classes there is a good correlation between absolute length of a bivalent and the frequency with which it is devoid of nodules, shorter bivalents more frequently lacking nodules than longer ones. 
Table XI

Observed and expected random distributions of nodules and chiasmata among the bivalents in Bombyx spermatocytes. ( $O$, observed frequency; $T$, theoretical frequency).

\begin{tabular}{lcrrrrrrrrr}
\hline \multirow{2}{*}{ Stage } & & \multicolumn{7}{c}{ Percent bivalents with n nodules (or chiasmata) } \\
\cline { 2 - 12 } & $\mathrm{n}=$ & 0 & 1 & 2 & 3 & 4 & 5 & 6 & 7 & $>7$ \\
\hline Late zygotene & $\mathrm{O}$ & 3 & 13 & 22 & 24 & 16 & 12 & 4 & 3 & 3 \\
& $\mathrm{~T}$ & 6 & 14 & 19 & 20 & 16 & 11 & 7 & 4 & 3 \\
\hline Early pachytene & $\mathrm{O}$ & 8 & 27 & 30 & 20 & 8 & 4 & 2 & 1 & 0 \\
& $\mathrm{~T}$ & 13 & 25 & 25 & 18 & 10 & 5 & 2 & 1 & 0 \\
\hline Mid pachytene & $\mathrm{O}$ & 2 & 39 & 31 & 18 & 8 & 1 & 1 & 0 & 0 \\
& $\mathrm{~T}$ & 15 & 27 & 26 & 17 & 9 & 4 & 1 & 1 & 0 \\
\hline Late pachytene & $\mathrm{O}$ & 2 & 23 & 32 & 26 & 8 & 5 & 2 & 2 & 0 \\
& $\mathrm{~T}$ & 9 & 21 & 25 & 20 & 13 & 7 & 3 & 1 & 1 \\
\hline Pachytene-diplotene & $\mathrm{O}$ & 4 & 30 & 19 & 12 & 21 & 11 & 1 & 2 & 0 \\
& $\mathrm{~T}$ & 8 & 19 & 24 & 20 & 14 & 8 & 4 & 2 & 1 \\
\hline Mid diakinesisa) & $\mathrm{O}$ & 2 & 18 & 43 & 21 & 14 & 0 & 2 & 0 & 0 \\
\hline Early diskinesisb) & $\mathrm{O}$ & $?$ & 14 & 35 & 36 & 11 & 3 & 1 & 0 & 0 \\
\hline Mid diakinesisb) & $\mathrm{O}$ & $?$ & 38 & 43 & 16 & 3 & 0 & 0 & 0 & 0 \\
\hline
\end{tabular}

a) $n$ equals the number of chiasmata.

b) Chiasma frequency based on light microscopical analysis of MAEDA (46).

\subsubsection{Distribution of chiasmata among bivalents}

In the two completely reconstructed mid diakinesis nuclei, two univalents and one bivalent with 6 chiasmata were revealed while the rest of the bivalents had from 1 to 4 chiasmata (Table XI). This distribution is very similar to that reported by MAEDA (46) at early diakinesis, but deviates somewhat from that reported for mid diakinesis (Table XI). This apparent discrepancy at mid diakinesis may primarily be due to differences in the criteria used for the stage determination between the light microscope analysis and the present analysis in combination with the limited number of nuclei reconstructed.

Although the data are insufficient for a reliable statistical analysis, a comparison of the distribution of recombination nodules during the mid pachytene to pachytene-diplotene period and the chiasma distribution at mid diakinesis demonstrates basic similarities: The number of homologues without recombination nodules or chiasmata is low, the vast majority of bivalents having from 1 to 4 recombination nodules or chiasmata. Only rarely do bivalents possess more than 4 nodules or chiasmata. This similarity of the distributions is consistent with the observation that recombination nodules participate in chiasma formation.

\subsubsection{Distribution of nodules along bivalents}

An analysis of the distribution of recombination and chromatin nodules within individual bivalents was not considered meaningful except for bivalents 1 and 2, due to the lack of reliable criteria for the classification of the remaining 26 bivalents. Idiograms, including the position of both types of nodules were, however, made for all late zygotene to pachytene-diplotene nuclei 


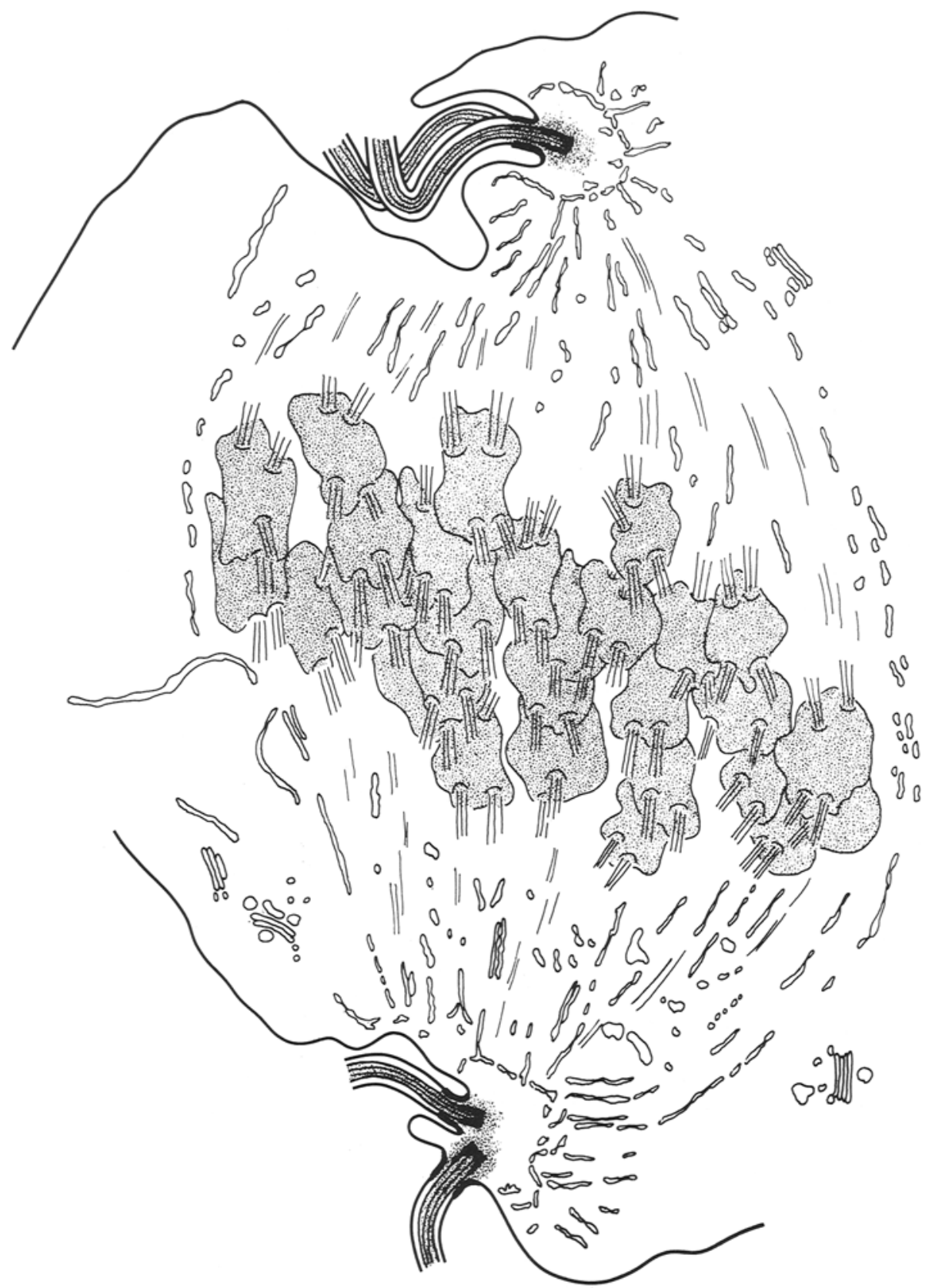

Figure 54. Semidiagrammatic reconstruction of a metaphase I cell (number 35). (Magnification approximately 9,000 times).

but did not reveal obvious patterns in the distributions of nodules along the individual bivalents. Clusters of nodules were not observed in specific regions and regions consistently depleted of nodules could not be identified either.
For bivalents 1 and 2, the length of each arm was adjusted to the mean length of that particular arm for each stage and the relative positions of the nodules were determined. The arms were then divided into $1 \mu \mathrm{m}$ segments and 

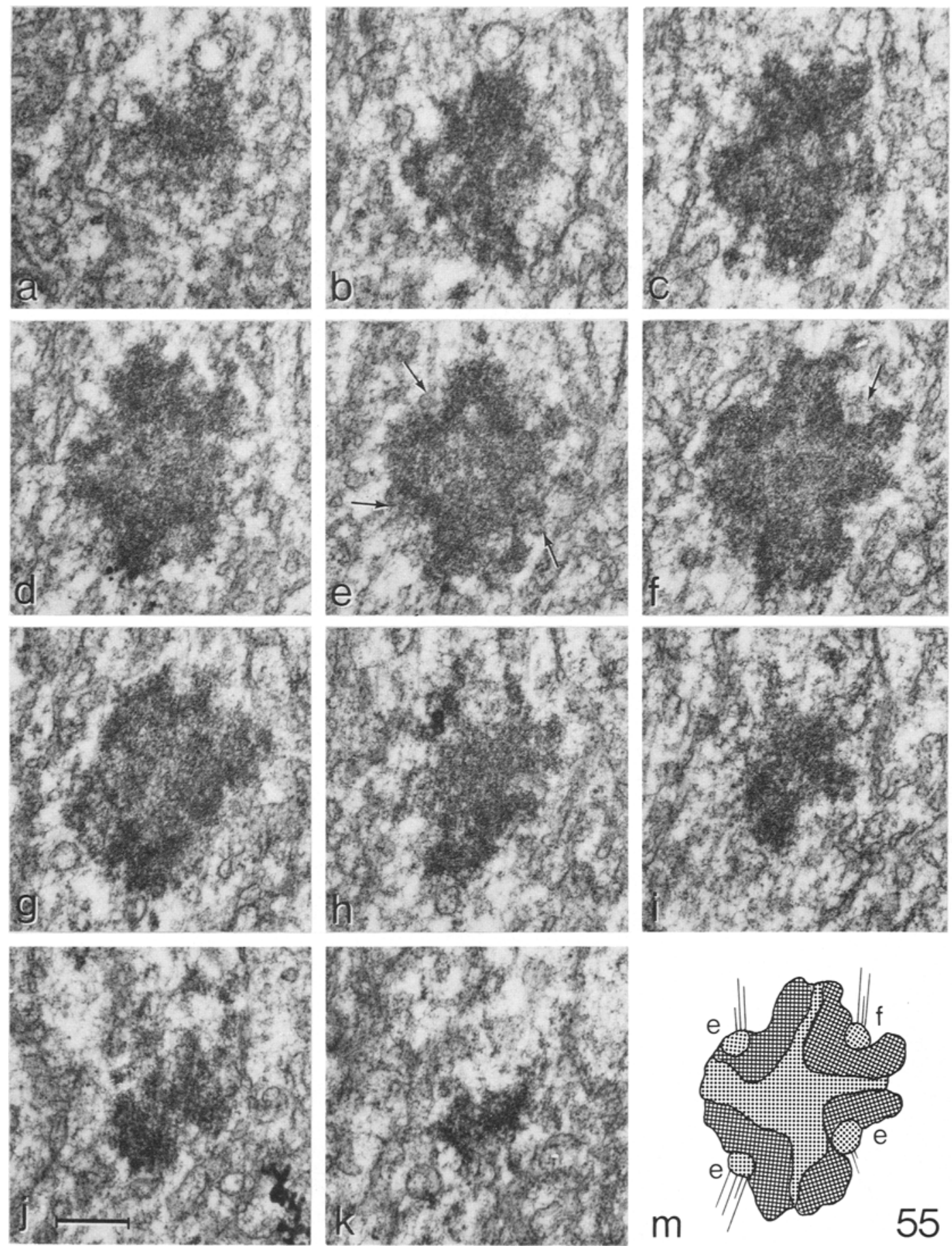

Figure 55. Eleven consecutive sections through a metaphase I bivalent. A reconstruction is shown in Figure $55 \mathrm{~m}$. The arrows in Figures 55e and $\mathrm{f}$ denote the position of the centromeres. (Bar $=0.5 \mu \mathrm{m}$ ). 


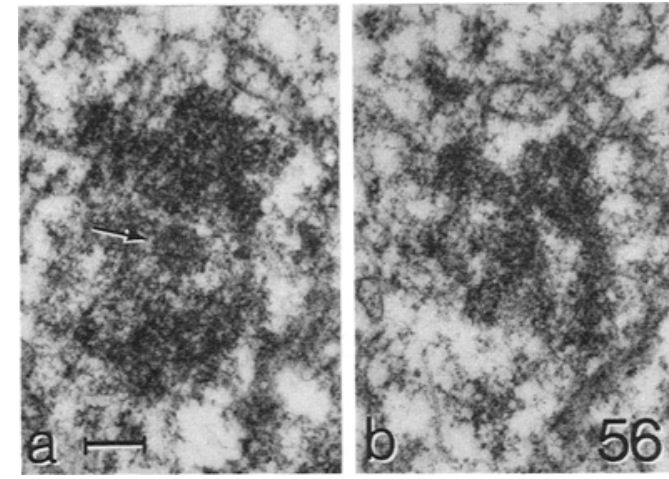

Figure 56. Two consecutive sections through a metaphase I bivalent. Note the circular structure (denoted by arrow) in Figure 56a. (Bar $=0.2 \mu \mathrm{m})$.

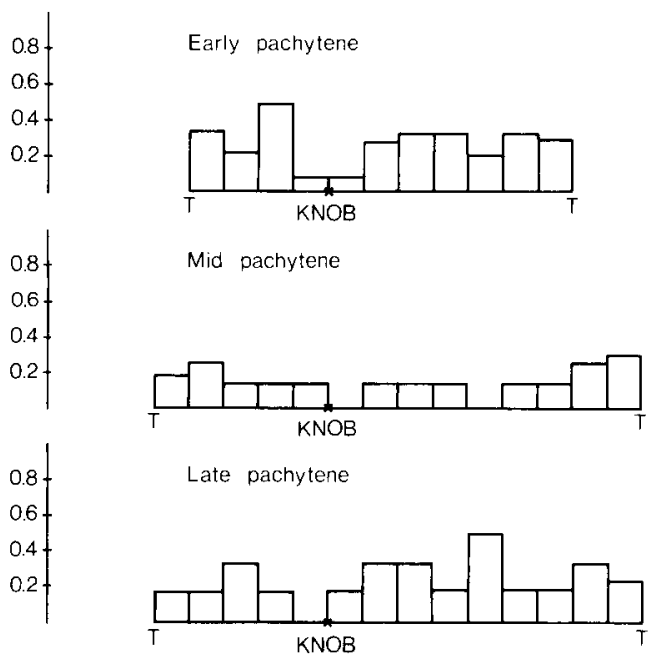

Figure 57. Histograms illustrating the distribution of nodules along bivalent $I$ at early, mid and late pachytene. The mean lengths of the bivalents divided into $1 \mu \mathrm{m}$ intervals are shown on the $\mathrm{X}$-axes and the mean number of nodules per segment on the $\mathrm{Y}$-axes. $T$ denotes position of the telomeres. the mean number of nodules in each segment calculated for each stage (Figures 57 and 58). (Data for bivalent 1 at late zygotene are not included, as this bivalent was only paired over $53 \%$ of its length). The number of observations was not sufficient for a statistical evaluation of the distribution of nodules, but the general impression one gets is that nodules are evenly distributed along the bivalents from late zygotene to the pachytene - diplotene transition, i.e., within a given nucleus, nodules are positioned at random along each bivalent.

In order to investigate this result in more detail, all neighbour nodule and nodule-telomere distances were measured and the distribution of these distances compared with the random distributions obtained from the computer simulation experiment as described in section 3.19.3.
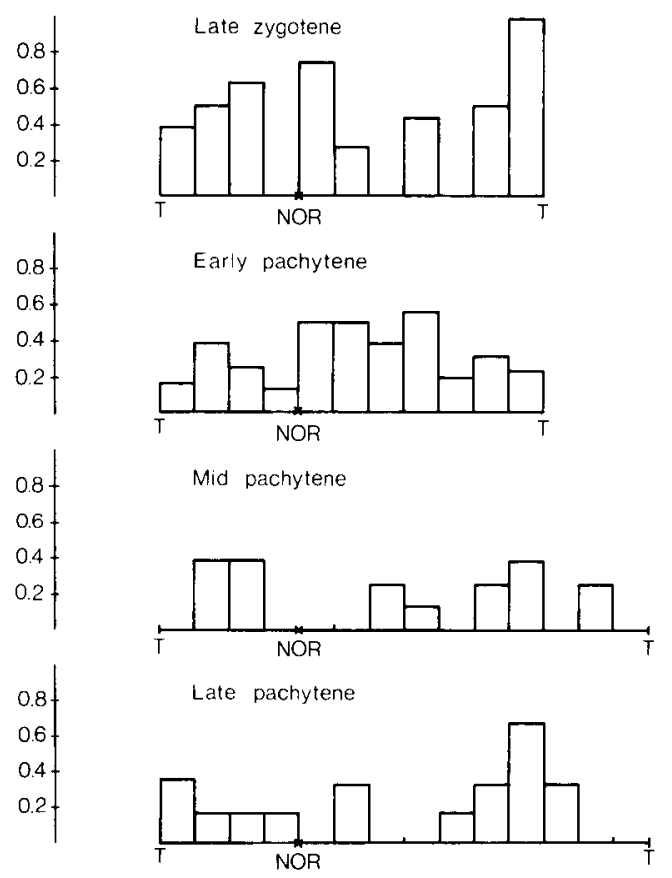

Figure 58. Histograms illustrating the distribution of nodules along bivalent 2 at late zygotene and early, mid and late pachytene. The mean lengths of the bivalents divided into $1 \mu \mathrm{m}$ segments are shown on the $\mathrm{X}$-axes and the mean number of nodules per segment on the $\mathrm{Y}$-axes. The mean number of nodules at late zygotene is corrected for incomplete pairing in individual nuclei by adding a number of nodules corresponding to that received by segments already paired in the nucleus. 


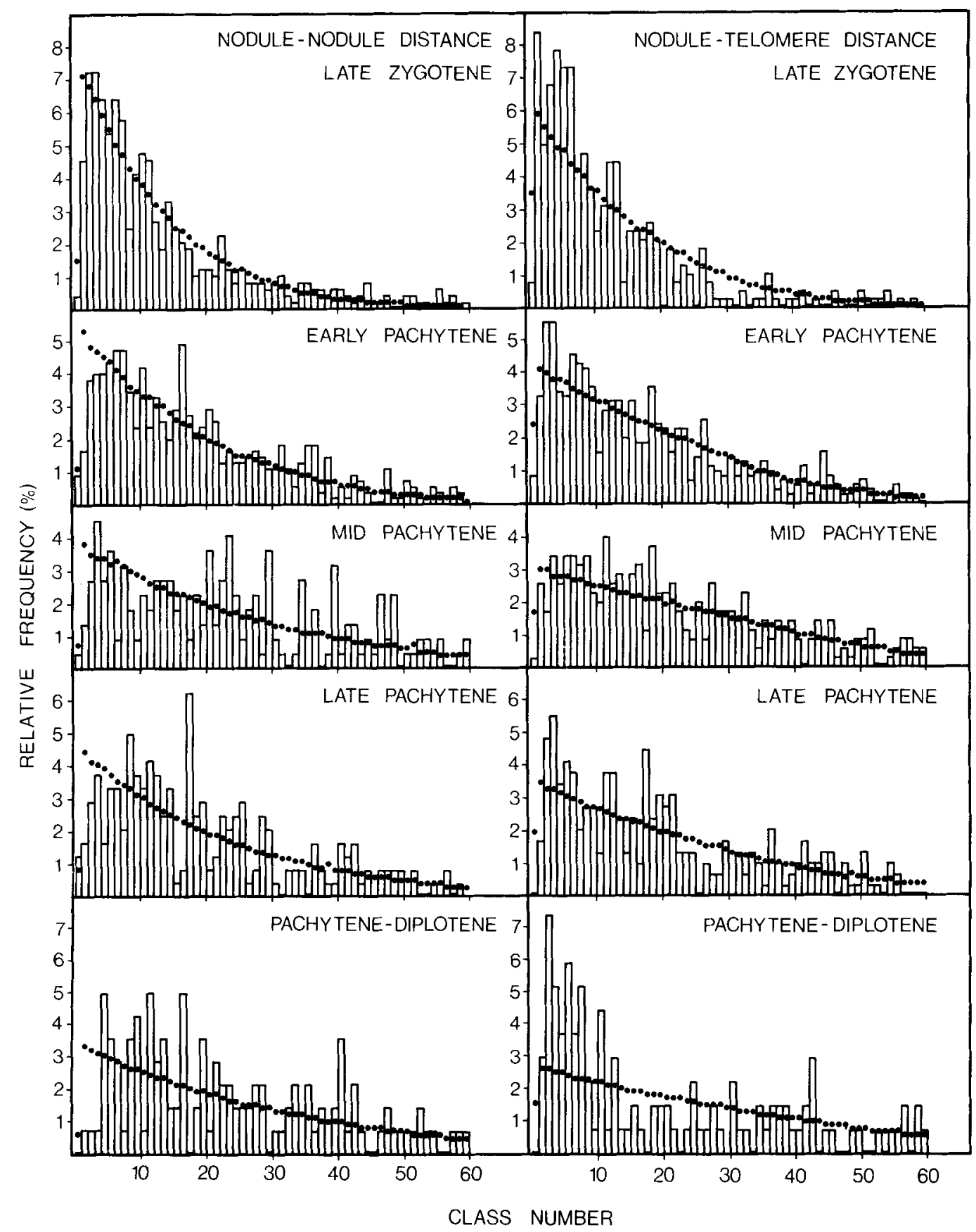

Figure 59. Histograms showing the distribution of nodule-nodule and nodule-telomere distances at late zygotene (LZ), early pachytene (EP), mid pachytene (MP), late pachytene (LP) and pachytene-diplotene (PD). The distances are expressed as relative frequencies of distances in $0.1 \mu \mathrm{m}$ classes from 0 to $6 \mu \mathrm{m}$. $(\mathrm{n}=483 \mathrm{LZ} ; 552 \mathrm{EP} ; 221$ MP, $241 \mathrm{LP} ; 141$ PD for nodule-nodule distances and $382 \mathrm{LZ} ; 02 \mathrm{EP} ; 349 \mathrm{MP} ; 288 \mathrm{LP}$; 136 PD for noduletelomere distances). The dotted lines are the expected random distributions of nodules along the bivalents. ( $\mathrm{n}=$ 505,305 LZ; 289,911 EP; 252,018 MP; 354,805 LP; 397,428 PD for nodule-nodule distances and 378,425 LZ; 333,249 EP; 320,887 MP; 361,532 LP; 368,408 PD for the nodule-telomere distances). For further details see sections 3.19.3. and 3.19.5. 
At late zygotene, the unpaired middle regions of partially paired bivalents were excluded by joining the two paired segments and all neighbour nodule distances extending across this point were not measured. Likewise, the computer was programmed to measure only the shortest nodule-telomere distance in bivalents with one nodule.

The observed and theoretical distributions for neighbour nodule and nodule-telomere distances are given in Figure 59. At late zygotene, there is a good correlation between the observed and the theoretical distributions, with a minor deficit of observed nodule-nodule distances in the range from 0 to $0.2 \mu \mathrm{m}$. In the following stages, this deficit of shorter distances $(0-0.5 \mu \mathrm{m})$ becomes more pronounced and is accompanied by an excess of longer distances in comparison with the theoretical distribution. This can be illustrated by calculating the observed and the theoretical frequencies in the interval from 0 to $0.5 \mu \mathrm{m}$. At late zygotene, $26 \%$ of all distances is found in this interval against an expectation of $28 \%$, while at early pachytene, $14 \%$ is observed and $20 \%$ expected. For mid and late pachytene, the figures are $12 \%$ and $12 \%$ observed against an expectation of $15 \%$ and $18 \%$ respectively. Finally, at the pachytene - diplotene transition, $7 \%$ is observed while $13 \%$ is expected, were nodules distributed randomly along the bivalents.

Apart from this minor deficit of shorter distances, the observed and the theoretical distributions are remarkably similar, and the deviations seen in the individual classes from mid pachytene to pachytene-diplotene possibly reflect the small class width and the low number of observations (Figure 59) rather than a true dissimilarity between the observed. and the theoretical random distribution.

The analysis of the nodule-telomere distances reveals more complex patterns when compared to a random distribution. At late zygotene and pachytene-diplotene, and to a lesser extent also at early and late pachytene, there is a clear excess of observed distances between 0 and $1.0 \mu \mathrm{m}$, whereas the correlation between observed and theoretical distributions is good at mid pachytene. The excess of nodules close to the telomere is at all four stages accompanied by a corresponding deficit of distances from 2.0 to $4.0 \mu \mathrm{m}$.

\section{DISCUSSION}

\subsection{General aspects of the meiotic prophase}

The present investigation, being based on three dimensional reconstruction of 82 nuclei divided into 15 well characterized substages, provides detailed information on the morphological changes of the nucleus from early zygotene to metaphase I. The temporal changes in the pattern of chromatin condensation described in previous sections are basically similar to those reported by KAWAGUCHI (38), its most characteristic feature being the complete decondensation of the chromatin initiated after pachytene and lasting to early diakinesis. During this period, the only condensed regions are those in the immediate vicinity of the chiasmata in accordance with KaWAGUCHI's description of the bivalents at diakinesis as: "...dünne Perlschnüre mit zwei bis vier Perlen«. The first contraction stage at mid - late zygotene described by KaWAGUCHI was not found in cells fixed for electron microscopy and is most likely a result of the fixation procedure used for light microscopy.

The changes in centriolar morphology and localization correlate well with the morphological changes of the nucleus and thus serve as additional characteristics of the individual stages. The pair of centrioles duplicate prior to early zygotene and remain within the organellar assembly outside the bouquet region until early pachytene. At mid pachytene, the two pairs of centrioles establish contact with the cell membrane and the growth of the flagellae is initiated. By mid diplotene, the proximal part of the centrioles comes in contact with the nuclear envelope and at early diakinesis the centrioles detach from the nuclear envelope and invaginations evolve at the former attachment sites. The position of these invaginations changes in parallel with the migration of the centriole pairs towards opposite poles at late diakinesis metaphase I. This pattern is consistent with that reported by FRIEDLÄNDER and W AHRMAN (20) in Bombyx spermatocytes.

The reconstructions of whole nuclei furthermore revealed an approximately 2.5 fold increase in nuclear volume between early zygotene and late diakinesis, predominantly in the early zygotene to late pachytene interval with only a minor additional increase during the later stages. 
The increase in nuclear volume is accompanied by a $25 \%$ increase in synaptonemal complex length from early to mid pachytene and by an additional $35 \%$ increase from late pachytene to pachytene-diplotene. Comparable changes in synaptonemal complex length have been found between early and mid-late pachytene in di- and triploid Bombyx oocytes $(63,65)$ and in human spermatocytes (66) whereas in tetraploid Bombyx oocytes, a decrease in length was revealed (68). In the ascomycetes Sordaria (86) and Neurospora (25) synaptonemal complex length increases between zygotene and pachytene while in Drosophila $(11,12)$, the complement length decreases during the first part of pachytene but again increases toward the end of pachytene. The functional significance of these apparently species specific variations in total synaptonemal complex length is so far unknown. It should be noted though that in Bombyx spermatocytes, the increase in length of the total complement correlated with changes in the ultrastructure of the synaptonemal complex, such as splitting of the lateral components and a more distinct central region. A similar situation is encountered in Drosophila where the decrease in length is accompanied by a thickening of the synaptonemal complex $(11,12)$.

\subsection{Chromosome pairing}

Chromosome pairing and formation of the synaptonemal complex follow in diploid Bombyx spermatocytes essentially the same general scheme as that reported for Bombyx oocytes (63) and human spermatocytes (66). Synaptonemal complex formation starts prior to the organization of continuous lateral components and with few exceptions, initiation is from telomeric regions. Throughout the pairing phase, the telomeres remain attached to the nuclear envelope within a restricted area of the membrane giving rise to a prominent chromosome bouquet. Both the attachment of the telomeres to the nuclear envelope and their subsequent congression possibly facilitate the initial matching of homologous chromosomes by restricting the freedom of telomere movements to a limited two dimensional space. The mechanism responsible for bringing together the homologous chromosomes is unknown. It is, however, of interest that
PARVINEN and SöDERSTRÖM (60) on the basis of time-lapse recordings of rat spermatocytes reported that nuclear rotation and chromosome movements are most intense during the period of chromosome pairing. Undoubtedly, these earlymid zygotene movements correlate with the process of chromosome alignment prior to synaptonemal complex formation as the movements cease by the end of zygotene and are absent throughout the remainder of the meiotic prophase. These observations indicate that the homologous are brought into proximity by random rather than by directed movements.

Following the initial matching of homologous telomeres, synaptonemal complex formation proceeds from telomere to telomere if not impeded by interlocking. Additional interstitial initiation of complex formation has only been observed in few cases and with one exception only between homologues involved in interlockings. For chromosomes 1, the knob appears to impose a restraint to complex formation as previously reported for chromosome 1 in tetraploid Bombyx oocytes (68) and for the centromeric heterochromatin of all bivalents in human spermatocytes (66). These local restraints may promote synaptonemal complex formation starting from both ends of the homologues.

The assessment of the specificity of pairing and synaptonemal complex formation is in Bombyx spermatocytes hampered by the fact that only four of the 56 chromosomes can be identified with certainty by morphological markers. In all eight late zygotene nuclei, the pairing of these chromosomes was strictly homologous. For the remaining 52 chromosomes, an evaluation of pairing specificity during zygotene has to be based upon less well-defined parameters, such as identity in length of the two chromosomes of each bivalent. As already pointed out in section 3.2., the gradual decrease in length from the longest to the shortest chromosome renders this criterium less valuable in chromosome identification. In no case were bivalents observed with one lateral component extending beyond the completed synaptonemal complex, and only in one case was a small interstitial synaptonemal complex segment found between apparently nonhomologous chromosomes as described in section 3.5.2. Hence, although in part based on circumstantial evidence, the conclusion is that 
the zygotene chromosome pairing and synaptonemal complex formation appears to be specific in Bombyx spermatocytes, as in all other organisms investigated so far.

The present analysis has added further evidence to the conclusion that interlockings are regular features of the zygotene chromosome pairing (69). The mean frequency of interlockings (chromosome and bivalent interlockings) in Bombyx spermatocytes amounts to four per nucleus, and all analyzed nuclei contained one or more interlockings. A smaller, but comparable, mean value of 0.7 interlockings per nucleus was observed in human spermatocytes, where $60 \%$ of the nuclei had one or more interlockings (66). In Bombyx oocytes, three of four analyzed nuclei contained interlockings, the mean frequency being one per nucleus (63). A second general result of this study is that interlockings are resolved by breakage and reunion of chromosomes or bivalents as previously described in human spermatocytes (66).

Interlocking has traditionally been considered to be rare in classical cytology, but as pointed out already in 1922 by GeLEI (22) and later confirmed by several others $(3,4,7,8,15,16$, $21,50)$, the congression of fortuitously distributed chromosomes may occasionally lead to interlocking of chromosomes and bivalents or as stated by GeLEI: ». . . kann daran kein Zweifel sein, dass in sehr seltenen Fällen auch ganz unlösbare Konjugationsstellungen durch Ineinanderhängen entstehen können.« (22). The commonly accepted notion that interlocking is a rare phenomenon may have its origin in the fact that observations in the light microscope of the entangled late zygotene chromosomes are met with difficulties as the whole nucleus is either included in one thick section or squashed, the latter process being especially fatal for the analysis of the spatial relationship of the chromosome complement of a zygotene nucleus. Furthermore, since most cytological studies have been and still are performed on squashed nuclei at the stages between late diplotene and metaphase $I$, the infrequent observation of interlockings is understandable as very few interlockings escape the resolution process. In Bombyx spermatocytes and oocytes $(63,65,68)$, interlockings have never been found at mid pachytene or later, whereas in human spermatocytes, four cases of unresolved interlockings were revealed in 43 early, mid and late pachytene nuclei $(29,66)$. Besides, the preservation of interlockings until diakinesis requires the presence of chiasmata in the segments on either side of the interlocking.

The possibility that interlockings may be resolved by chromosome breakage and reunion has previously been inferred by GeLEI (22): "... oder wenn sie (the pairing) um jeden Preis geschehen muss, ein Faden in dem Hin- und Herziehen reissen und die Ringe dadurch frei werden, wenn nicht erst die Reifeteilung die Lage löst. ...«. This possibility has later been considered and rejected by Buss and HENDERSON $(7,8)$. The scarcity of interlockings in the diplotene - metaphase I interval in normal diploid organisms has generally been attributed to a hypothetical feature of the initial stage of pairing, namely that prior to the actual pairing and synaptonemal complex formation, the homologues are already juxtapositioned. It is believed that this premeiotic alignment occurs at a stage where the chromosomes are in a condensed state and that the alignment acts to minimize the risk of interlocking if the initial matching of the homologues is maintained into the meiotic prophase $(6,18,70)$ (for example, as suggested by MAGuire, 47, by establishment of elastic connections between homologous chromosomes). Evidently, this argument is no longer relevant.

The very low frequency of chromosomal aberrations, such as chromosome or bivalent fragments or translocations at pachytene and later stages, furthermore shows that in both human and Bombyx spermatocytes, the breakage, reunion and repair of the broken ends are precisely controlled processes fundamentally different from, for example, the breakage fusion - bridge cycle in maize heterozygous for a paracentric inversion described by MCCLINTOCK (51) where the breakage is caused by physical tension. A controlled breakage may be a necessary prerequisite for the precise and specific repair mechanism capable of joining not only broken ends of one of the two homologues but also the broken ends of bivalents (66). The former may be a more simple process as the intact other homologue, by specific pairing with the broken one, may facilitate the juxtaposition of the broken ends. The latter type of break 
imposes a more difficult problem as in this case, bivalent continuity is disrupted. In this context, it is of interest that a new type of DNA topoisomerase isolated from E. coli cells is able, in the presence of ATP, to convert knotted DNA circles into unknotted circles by a concerted cleavage and rejoining of both strands of the DNA double helix (45). Similar enzyme activities are present in extracts from Drosophila eggs, Xenopus eggs and mammalian cells in tissue culture. It is conceivable that a similar DNA topoisomerase is involved in the breakage and reunion of chromatids during the resolution of interlockings. If so, the discontinuities of the lateral components and synaptonemal complexes may not reflect discontinuities of the DNA, and although high magnification micrographs of the broken lateral components and synaptonemal complexes generally do not reveal chromatin connections between the broken ends which may be separated by several $\mu \mathrm{m}$ (see Figures 14 and 15), the existence of DNA continuity cannot be excluded.

In the grasshopper, Locusta migratoria, heat treatment has been shown to induce bivalent interlockings in 5 to $11.3 \%$ of the nuclei (8). The period most sensitive to induction was reported to coinside with the premeiotic S-phase or to be even earlier. In most cases, only the longest chromosomes were involved in the induced interlockings. In view of the observations in human and Bombyx spermatocytes it is likely that the induction of interlockings by heat treatment in Locusta, rather reflects the inactivation of the break-repair system than being due to disturbance of a premeiotic alignment as favored by the authors. BARBER (3), on the other hand, did not observe an increase in the frequency of interlockings upon heat treatment in Fritillaria, while colchicine treatment was effective in producing interlockings (in up to $25 \%$ of the examined bivalents). Whether the effect of colchicine in Fritillaria results from disturbance of the zygotene pairing process or reflects the inhibition of the break-repair system is uncertain.

It should be noted that inaccuracy of the break-repair system is a potential source of chromosomal rearrangements as well as of sister chromatid exchanges and aberrant wcrossing over « involving all four chromatids at a given site (the latter in cases where a $180^{\circ}$ rotation of the broken end of one bivalent fragment relative to the other has occurred prior to reunion and repair of the broken ends). Finally, occasional failure of the repair system may lead to the formation of chromosome fragments as seen in both humans and Bombyx $(29,65,66,68,69)$.

\subsection{Recombination nodules}

\subsubsection{General aspects}

The evidence for a relationship between recombination nodules, crossing over and gene conversion has recently been reviewed $(13,53$, 69) and will not be repeated here. As discussed in section 4.4., this relationship has gained direct support by the morphological continuity between recombination nodules and chiasmata. In the following, it is assumed that the presence of a recombination nodule in the central region of the synaptonemal complex is a necessary although not sufficient condition for the occurrence of genetic recombination.

Small recombination nodules first appear in Bombyx spermatocytes at early zygotene simultaneously with the initiation of synaptonemal complex formation. During zygotene, the number of both small and larger recombination nodules increases and by late zygotene the mean number of nodules amounts to 91 per nucleus. Assuming that unpaired regions upon pairing will have the same probability for receiving a nodule as already paired regions, 103 recombination nodules per nucleus are expected. Between late zygotene and early pachytene, this number decrease by $41 \%$. Comparable decreases in the total number of nodules between late zygotene and early pachytene have been reported in human spermatocytes (66) and in Schizophyllum (10). In human spermatocytes, the decrease amounts to $48 \%$ and in Schizophyllum to $41 \%$. It has been suggested that this drastic and fairly constant reduction in the number of recombination nodules somehow relates to the occurrence of reciprocal and nonreciprocal recombination $(66,69)$. Generally, a unitary mechanism is believed to account for both types of recombination, the outcome being dependent only upon how the proposed intermediate - the half chiasma - is resolved $(27,56)$. Hence, elimination of a given recombination nodule after 
zygotene may signify termination of a nonreciprocal recombination event, whereas reciprocal exchanges require that the recombination nodule remains attached to the central region of the synaptonemal complex at the site of the crossover (cf. section 4.4.). The 40 to $50 \%$ reduction in the number of recombination nodules is in accordance with the general notion that despite substantial differences among different loci, reciprocal and nonreciprocal exchanges are as a whole almost equally frequent when measured as the frequency of gene conversion with and without exchange of flanking markers $(14,27$, $34,42,56)$. This concept has, however, been challenged $(61,72)$ and new estimates for yeast gave only $35 \%$ conversion events associated with exchange of flanking markers (19). Exactly how the 40 to $50 \%$ reduction of the number of recombination nodules matches the genetic data is at present unclear. It may indicate that conversion without flanking marker exchange also occurs at later stages, but considering the present evidence, further speculations appear premature.

The minimum number of recombination nodules is in Bombyx spermatocytes found at mid pachytene. By late pachytene, the number of nodules increases to slightly above the early pachytene level. A similar although more drastic decrease in the number of nodules takes place between early and mid pachytene in human spermatocytes. BERTHELSEN et al. (5) reported in three mid and late pachytene nuclei a mean of 15 nodules per nucleus but also found 10 to 25 dense structures bridging the central region of the synaptonemal complex similar to those reported by Moens (54) in the rat. The bridgelike structures were more readily identified in the one very late pachytene nucleus.

In a recent paper, SolaRI (74) reported that in human spermatocytes, a mean of 46 bridge-like structures was present throughout pachytene. The distribution of these bridges was very similar to the distribution of recombination nodules in sectioned material $(5,66)$ and it is conceivable that the bridges represent recombination nodules or their derivatives.

\subsubsection{Distribution of nodules and chiasmata among bivalents \\ The present investigation has shown, by}

comparing the observed nodule distribution among bivalents with a random distribution, that at late zygotene and, although less pronounced, also at early pachytene, recombination nodules are distributed nearly randomly among the bivalents of a nucleus. From early to late pachytene this distribution changes by association of nodules preferentially to bivalents without nodules but also to some extent to bivalents with 1 and 2 nodules. This modified distribution remains unaltered during the evolution of chiasmata at diplotene, since at mid diakinesis, the distribution of chiasmata closely resembles the late pachytene nodule distribution. A comparison between the observed and expected random distributions of nodules at late zygotene and early pachytene in human spermatocytes (31) revealed the same pattern as that seen in Bombyx spermatocytes. At present, it is not known whether a modification of the nodule distribution occurs also in human spermatocytes after early pachytene.

In Bombyx spermatocytes, $2 \%$ of the bivalents are without nodules at late pachytene. If this implies that crossing over will not occur in these bivalents, then nondisjunction is expected in $1 \%$ of the bivalents, resulting in a maximum of $28 \%$ unbalanced gametes. Data for nondisjunction in Bombyx spermatocytes are scarce and do not permit a direct comparison of the estimated level of nondisjunction. This level appears, however, unrealistically high which, at least in part, may be due to the difficulty in identifying recombination nodules particularly at the pachytene-diplotene transition. Nodules may, for example, have escaped attention in cases where the plane of sectioning passes through a nodule or where a nodule is included in one of the sections adjacent to that including the synaptonemal complex segment. The number of bivalents without nodules may therefore be overestimated.

Comparable data for the distribution of recombination nodules among bivalents are not available for other organisms, but in nine Drosophila oocyte nuclei selected for having more than four spherical nodules, which are believed to be involved in reciprocal exchange, a significant difference in the number of nodules per chromosome arm was found when compared to a Poisson distribution (12). It is, however, 
not clear whether the distribution would follow a Poisson distribution with all analyzed nuclei included, i.e., also nuclei with less than four nodules. In contrast, small ellipsoid nodules, which in Drosophila are present at a slightly earlier stage and believed to mediate nonreciprocal exchange, exhibit a good correlation with a random distribution when nuclei with less than four ellipsoid nodules are excluded (12). It is again uncertain how the distribution would be affected if the remainder of the analyzed nuclei were included.

The above data are in agreement with the general contention that the distribution of crossovers and chiasmata among bivalents probably are the result of two processes: In most organisms, the mean number of crossovers and chiasmata per bivalent are directly proportional to the length of the bivalent (16), i.e., reflect a random distribution within the individual nucleus. In addition, mechanisms exist which appear to promote the occurrence of at least one crossover per bivalent and which also impose an upper limit to the number of exchanges per bivalent (16).

The breakdown of the latter mechanism by mutation is illustrated by JoNes (36), who in a mutant of rye observed a normal number of chiasmata randomly distributed among the bivalents eventually leading to precocious separation of several pairs of homologues. It is likely that this mutant specifically blocks the secondary redistribution of recombination nodules.

In Bombyx spermatocytes, the shift in nodule distribution is accompanied by changes in the ultrastructure of the synaptonemal complex (the lateral components become double and the central region appears more distinctly cross striated) but it has not been possible to correlate these morphological changes with the presence/ absence of recombination or chromatin nodules and the mechanisms underlying the modification of the nodule distribution are so far unknown.

\subsubsection{Distribution of nodules along bivalents}

Genetic control of the distribution of exchanges and chiasmata within bivalents is well documented in many organisms $(2,16,26,87)$. The control may be exerted at three levels: 1) An absolute regulation where larger chromosome regions are either excluded from participating in crossing over, as is generally the case for heterochromatin, particularly the centromeric heterochromatin, or have increased probability of crossing over and chiasma formation as is often the case for regions near the telomeres. 2) A relative regulation which somehow decreases the probability for two crossovers in the vicinity of each other (positive interference). 3) A specific control of recombination in individual genes (14, 43).

The present data from Bombyx clearly demonstrate that basically, nodules are positioned at randon along the individual bivalents, with only a minor tendency of neighbour nodules to be farther apart than expected from a random distribution. These results are remarkably similar to those previously reported for human spermatocytes (66), but are at variance with the situation reported in Drosophila $(12,13)$. In chromosome arms with two nodules, a mean neighbour nodule distance of $33 \%$ of the arm length is expected if nodules are placed at random along the arms. Three observed neighbour nodule distances for ellipsoid nodules gave in Drosophila oocytes (12) a mean distance of $30 \%$ of the arm length while the mean of six distances between spherical neighbour nodules amounted to $51 \%$. Four distances between ellipsoid - spherical neighbour nodules gave a mean of $50 \%$ of the arm length. Based on these data it was concluded that ellipsoid neighbour nodules do not exhibit positive interference while ellipsoid - spherical neighbour nodules as well as spherical neighbour nodules do.

Positive interference has been demonstrated in Bombyx spermatocytes for the $\mathrm{Z}$ chromosome and for chromosome 5 (78), but it is at present not clear how this phenomenon is reflected in the distribution of recombination and chromatin nodules. One major problem in this context is that little is known about the physical distances (in terms of synaptonemal complex length for example) over which interference operates. Also the general assumption upon which the calculation of the expected number of double crossovers is based, namely that adjacent chromosome regions of equal size also will have equal probability for crossing over, may require reconsideration. The total number of exchanges per nucleus is limited which will lead to a 
gradual decrease of the probability for additional exchanges events as the "pool of nodules" is exhausted.

On the basis of the observations in Drosophila, CARPENTER (13) proposed that the information for the placement of a nodule is embodied in the nodule. In view of the nearly random distribution of nodules it is our impression that this is not the case in human and Bombyx spermatocytes. More likely, the distribution is the result of a fairly constant total number of nodules available in combination with variations in the rate of synaptonemal complex formation (69). Subsequently, the resultant distribution is modified either by redistribution of nodules or by deposition of additional nodules. As pointed out in section 3.10., attempts to distinguish separate classes of recombination nodules have failed in Bombyx spermatocytes. Intermediate forms between small and large ones are frequent and the overall impression is that the larger nodules evolve from the smaller type. The observation that smaller nodules are more frequent at late zygotene implies that the decrease in total number of nodules between zygotene and pachytene primarily affects the smaller nodules. The main period of nonreciprocal exchanges may thus be prior to crossing over.

\subsection{Chiasmata}

The maximum size and density of recombination nodules are attained at mid pachytene in Bombyx spermatocytes. Simultaneously, an increasing fraction of the recombination nodules becomes larger and more irregular in shape probably in part by association of chromatin to the nodules. These chromatin nodules increase both in size and in number during late pachytene, and at early diplotene nearly all nodules are of this type. Initiation of synaptonemal complex degradation is at pachytene-diplotene accompanied by decondensation of the bulk of the chromatin culminating at mid diplotene where only about 60 major and a few minor domains of codensed chromatin remain. The major condensations are in most cases associated with remnants of the synaptonemal complex and the chromatin fills and partly surrounds the central region of the complex. Frequently, these synaptonemal complex remnants contain an electron dense core reminiscent of a recombination nodule. At late diplotene, the major condensations consist of two regions of condensed chromatin bridged by a circular structure 120$160 \mathrm{~nm}$ in diameter and composed of a dense core surrounded by a ring of about the same density, the center and the ring being connected by thin fibers. The fine structure of the circular components remains unchanged until mid diakinesis. From the diplotene - diakinesis transition onward the circular component is frequently surrounded by a chromatin bridge which combines two regions of condensed chromatin. Complete reconstructions at mid diakinesis reveal that these chromatin bridges together with the circular components constitute the chiasmata. At late diakinesis, the number of circular components decreases and at metaphase I, very few remain. The bivalents condense at these stages into more or less spherical bodies and chiasmata can no longer be identified.

The number of chiasmata is fairly constant from mid diplotene to mid diakinesis and the mean number of 58 per nucleus is very similar to the mean number of chromatin nodules at pachytene-diplotene and early diplotene. Furthermore, this value is approximately the same as the number of chiasmata at early and mid diakinesis reported from the light microscopic investigation by MAEDA (46). Finally, the distribution of chiasmata among the bivalents at mid diakinesis closely resembles the distribution of nodules during the latter part of pachytene as well as the distribution of chiasmata as determined by light microscopy (46). These observations are in agreement with a continuous morphological evolution of chiasmata in bivalent regions which during pachytene contains recombination nodules. Altogether these results suggest that in the male silkworm, recombination nodules in addition to their role in crossing over, together with retained fragments of the synaptonemal complex, also function in the establishment and maintenance of stable chromatin bridges chiasmata - and that this function of recombination nodules is accompanied by the formation of a complex circular structure.

In the ascomycete, Neottiella, pieces of synaptonemal complex are retained between the homologues at diplotene. These pieces are at diakinesis replaced by apparently circular (or 
spherical) chromatin bridges, not unlike those seen in Bombyx spermatocytes. On the basis of these observations, WESTERGAARD and voN WETTSTEIN $(82,83)$ proposed that the synaptonemal complex fragments and the subsequent chromatin bridges were present at sites where crossing over had occurred and that both represent chiasmata. Similarly, SolarI (73) reported that in mouse spermatocytes lateral components remain paired through a piece of modified central region at diplotene, and also he observed that the remnants of the central region were later replaced by chromatin bridges. Retained synaptonemal complex fragments have furthermore been observed at diplotene in Zea mays (23), Neurospora (25), Ascaris (44) and in Sordaria (86), but only in Neurospora, Ascaris and Sordaria were recombination nodules found in the retained pieces. Based on three dimensional reconstructions in Sordaria, ZICKLER (86) reported a number of synaptonemal complex fragments about equal to the number of chiasmata, most of the fragments containing recombination nodules. In all these species, (Ascaris being a possible exception) the retained synaptonemal complex fragments with or without recombination nodules disappeared before diakinesis.

These reports are as a whole consistent with the observations in Bombyx spermatocytes that retained synaptonemal complex fragments and the derivatives of recombination nodules, the chromatin nodules, play a role in organization of chiasmata. It is likely that both recombination nodules and synaptonemal complex fragments are generally required for chisma organization.

The observation of retained synaptonemal complex fragments in metaphase I bivalents of the grasshoppers $(17,52,55)$, the pigeon (59) and human spermatocytes $(67,75)$ is more difficult to interpret. The fragments may represent auxillary chiasma structures (32) equivalent to the circular component of Bombyx spermatocytes or may serve as an additional safety device which, in combination with chromatin chiasmata originating from exchange of nonsister chromatids, ensure a regular disjunction. Finally, Moens (53) and Moens and ChurCH (55) proposed that such remnants serve in chiasma maintenance by holding together the sister chromatids. Evidently, more work involving reconstructions of nuclei from the stages between late pachytene and metaphase I is required before definite conclusions can be made concerning the significance of the synaptonemal complex fragments in these organisms.

The presence of the circular chiasma component from late diplotene to mid diakinesis in Bombyx spermatocytes indicates that a chiasma remains at the site of crossover until late diakinesis. Similar conclusions have recently been made from observations on grasshopper (81), mouse $(37,62)$ and Armenian hamster (1). Incorporation of bromodeoxyuridine at the penultimate S-phase, resulting in differential staining of the substituted and the unsubstituted, chromatids unequivocally demonstrated that also in these organisms, chiasmata remain at their original positions up to mid diakinesis as evidenced by the absence of exchange of labelled chromatids.

That factors other than the circular chiasma component and the flanking condensed chromatin may be operative in chiasma maintenance is implicit in the proposal by $\operatorname{MaGUIRE}(48,49)$ that chiasma maintenance is due to the cohesion between sister chromatids. The present evidence is, however, insufficient for the evaluation of the role of sister chromatid cohesion in chiasma maintenance.

\subsection{Metaphase I}

The present analysis has revealed in each metaphase I bivalent four localized centromeres, two-by-two facing opposite spindle poles. This observation is at variance with the general concept that Bombyx, as other Lepidoptera species, has nonlocalized centromeres $(58,79)$, but is in accordance with the presence of apparently localized centromeres at metaphase I in Bombyx oocytes (64). The differences in chromosomal orientation between meiotic and mitotic metaphases reported by MURAKAMI and ImaI (58) may be relevant in this context: At mitosis the chromosomes orient and separate with their long axis parallel to the equatorial plane as expected for holocentric chromosomes, while at meiosis, the homologues orient and move toward opposite poles with their long axis at right angle to the equatorial plane. This suggests a basic difference in the behaviour of the 
centromeres in the two types of divisions, the centromere being nonlocalized in mitosis and localized in the first meiotic division (although not morphological distinguishable from the rest of the chromatin at stages prior to metaphase I).

On the other hand, FRIEDLÄNDER and W AHRMAN (20) claimed that metaphase I bivalents of Bombyx spermatocytes possessed nonlocalized centromeres, but according to their own description the staging was not unambigous. The detailed analysis of centromere behaviour in mitotic and meiotic divisions by HuGHES-SCHRADER and SCHRADER (33) in Hemiptera clearly demonstrates that a basic difference in the behaviour of the centromere at the two types of division can occur. In three species of Hemiptera, the centromere at mitotic metaphase appeared nonlocalized as evidenced by an orientation of the chromosomes parallel to the equatorial plane, both in the metaphase plate and during separation of chromatids at anaphase. In contrast, the centromere activity in bivalents at metaphase I of meiosis was restricted to one telomere region per chromosome resulting in an orientation of the bivalents parallel to the pole-to-pole axis as is observed in Bombyx spermatocytes (58).

As pointed out by Hughes-Schrader and SCHRADER (33), incomplete chiasma elimination (terminalization) prior to metaphase I may in organisms with holocentric chromosomes lead to difficulties in achieving a regular disjunction whereas this would not be the case for mitotic divisions. Hence, the meiosis specific modification of otherwise holocentric chromosomes, leaving only one active centromere region per chromatid, possibly represents an evolutionary adaptation of the diffuse centromere to the problem of meiotic segregation.

Inactivation of most of the centromere activity at meiosis may also account for the small number of microtubules observed in the present study in otherwise fully organized metaphase I plates. It is conceivable that the formation of the spindle shaped membrane compartments around each bivalent somehow compensate for the scarcity of microtubules by facilitating the movement of the homologues toward opposite poles at anaphase I.

\section{ACKNOWLEDGEMENTS}

It is with great pleasure that we thank Dr. H. AKaI, Sericultural Experiment Station, Tsukuba, Japan, Dr. V. N. Vereiskaya and the late Professor B. L. Astaurov, Institute of Developmental Biology, Academy of Sciences USSR, Moscow for supplying us with the material on which this work is based. We also wish to thank professor D. VON WETTSTEIN for his support throughout the course of this investigation and for critically reviewing the manuscript. The expert technical assistance of J. SAGE, N. RASMUSSEN and A. STEINHOLTZ is gratefully acknowledged. Finally, we thank cand. stat. L. SeJERSEN for her assistance in constructing the program for the computer simulation.

The work was supported by grant 202-76-1 BIO DK from the Commission of the European Communities.

\section{REFERENCES}

1. Allen, J. W.: BrdU-characterization of late replication and meiotic recombination in Armenian hamster cells. Chromosoma (Berl.) 74, 189-207 (1979)

2. Baker, B. S., A. T. C. Carpenter, M. S Esposito, R. E. Esposito \& L. SAndler: The genetic control of meiosis. Ann. Rev. Genet. 10, 53-134 (1976)

3. Barber, H. N.: The experimental control of chromosome pairing in Fritillaria. J. Genet. 43, 359-374 (1942)

4. BèLAR, K.: Die Cytologische Grundlagen der Vererbung. In: Handbuch der Vererbungswissenschaft. E. Baur \& M. Hartmann, eds., Verlag Gebrüder Borntraeger, Berlin, Vol. $1412 \mathrm{pp}$ (1928)

5. Berthelsen, J. G., M. Føgh \& N. E. SkakkeBEK: Electron microscopical analysis of meiotic chromosomes from human spermatocytes during and after treatment with steroid hormones. Carlsberg Res. Commun. 45, 9-23 (1980)

6. Brown, W. V. \& S. M. Stack: Somatic pairing as a regular preliminary to meiosis. Bull. Torrey. Bot. Club. 95, 369-378 (1968)

7. Buss, M. E. \& S. A. Henderson: Induced bivalent interlocking and the course of chromosome synapsis. Nature New Biol. 234, 243-246 (1971)

8. Buss, M. E. \& S. A. Henderson: The induction of orientational instability and bivalent interlocking at meiosis. Chromosoma (Berl.) $35,153-183$ (1971) 
9. Byers, B. \& L. Goetsch: Electron microscopic observations on the meiotic karyotype of diploid and tetraploid Saccharomyces cerevisiae. Proc. Nat. Acad. Sci. USA 72, 5056-5060 (1975)

10. Carmi, P., P. B. Holm, S. W. Rasmussen, J. SAGE \& D. ZICKLER: The pachytene karyotype of Schizophyllum commune analyzed by three dimensional reconstructions of synaptonemal complexes. Carlsberg Res. Commun. 43, 117132 (1978)

11. Carpenter, A. T. C.: Electron microscopy of meiosis in Drosophila melanogaster II. The recombination nodule - a recombination associated structure at pachytene? Proc. Nat. Acad. Sci. USA 72, 3186-3189 (1975)

12. Carpenter, A. T. C.: Synaptonemal complex and recombination nodules in wild type Drosophila melanogaster females. Genetics $92,511-$ 541 (1979)

13. Carpenter, A. T. C.: Synaptonemal complex and recombination nodules in recombination deficient mutants of Drosophila melanogaster. Chromosoma (Berl.) 75, 259-292 (1979)

14. Catcheside, D. G.: The genetics of recombination. In: Genetics Principles and Perspectives. K. R. Lewis \& B. John eds. Edward Arnold, London Vol. $2172 \mathrm{pp}$ (1977)

15. Dark, S. O. S.: Meiosis in diploid and tetraploid Paeonia species. J. Genet. 32, 353-372 (1936)

16. Darlington, C. D.: Recent advances in cytology. 2nd ed. Churchill, London 67I pp (1937)

17. EsPonda, P. \& D. B. KRIMER: Development of the synaptonemal complex and polycomplex formation in three species of grasshoppers. Chromosoma (Berl.) 73, 237-245 (1979)

18. Feldman, M.: The effect of chromosomes 5B, 5D and 5A on chromosomal pairing in Triticum aestivum. Proc. Nat. Acad. Sci. USA 55, $1447-$ 1453 (1966)

19. Fogel, S., R. Mortimer, K. Lusnak \& F. TAVARES: Meiotic gene conversion: A signal of the basic recombination event in yeast. Cold Spring Harbor Symp. Quant. Biol. 43, 13251341 (1979)

20. Friedi.̈.nder, M. \& J. Wahrman: The spindle as a basal body distributor. A study in the meiosis of the male silkworm moth, Bombyx mori. J. Cell Sci. 7, 65-89 (1970)

21. Gairdner A. E. \& C. D. Darlington: Ringformation in diploid and polyploid Campanula persicifolia. Genetika 31, 113-150 (1931)

22. Gelel, J.: Studien über die Oogenese des Dendrocoelum lacteum. Arch. f. Zellforsch. 16 , 88-169 (1922)

23. Gilues, C. B.: An ultrastructural analysis of chromosal pairing in maize. Compt. Rend. Trav. Lab. Carlsberg 40, 135-161 (1975)

24. Gillies, C. B.: Synaptonemal complex and chromosome structure. Ann. Rev. Genet. 9, 91109 (1975)

25. Gillies, C. B.: The relationship between synaptonemal complexes, recombination nodules and crossing over in Neurospora crassa bivalents and translocation quadrivalents. Genetics 91, 1-17 (1979)

26. Golubovskaya, I. N.: Genetic control of meiosis. Int. Rev. Cytol. 58, 247-290 (1979)

27. Holliday, R.: Recombination and meiosis. Phil. Trans. R. Soc. London B. 277, 359-370 (1977)

28. HоLm, P. B.: Three dimensional reconstruction of chromosome pairing during the zygotene stage of meiosis in Lilium longiflorum (Thunb.) Carlsberg Res. Commun. 42, 103-151 (1977)

29. Holm, P. B. \& S. W. Rasmussen: Human meiosis I. The human pachytene karyotype analyzed by three dimensional reconstructions of the synaptonemal complex. Carlsberg Res. Commun. 42, 283-323 (1977)

30. Holm, P. B. \& S. W. Rasmussen: Human meiosis III. Electron microscopical analysis of chromosome pairing in an individual with a balanced translocation $46, \mathrm{XY}, \mathrm{t}(5 \mathrm{p}-; 22 \mathrm{p}+)$. Carlsberg Res. Commun. 43, 329-350 (1978)

31. Holm, P. B. \& S. W. Rasmussen: Chromosome pairing, crossing over, chiasma formation and disjunction as revealed by three dimensional reconstructions. In: International Cell Biology 1980-1981, G. Schweiger ed., Springer Verlag, Berlin, Heidelberg \& New York, pp 194-204 (1981)

32. Holm, P. B., S. W. Rasmussen \& D. von WettSTEIN: The possible contribution of electron microscopy to the understanding of the mechanism of non-disjunction in man. Mutation Res. 61, 115-119 (1979)

33. Hughes-Schrader, S. \& F. Schrader: The kinetochores of the Hemiptera. Chromosoma (Berl.) 12, 327-350 (1961)

34. Hurst, D. D., S. Fogel \& R. K. Mortimer: Conversion-associated recombination in yeast. Proc. Nat. Acad. Sci. USA 69, 101-105 (1972)

35. JansSENS, F. A.: Spermatogénèse dans les Bactraciens. V. La théorie de la chiasmatypie. Nouvelles interpretation des cineses de maturation. Cellule 25, 387-411 (1909)

36. JoNEs, G. H.: The control of chiasma distribution in rye. Chromosoma (Berl.) 22, 69-90 (1967)

37. KaNDA, N. \& H. KaTO: Analysis of crossing over in mouse meiotic cells by $\mathrm{BrdU}$ labelling 
technique. Chromosoma (Berl.) 78, 113-121 (1980)

38. KaWAGUCHI, E.: Zytologische Untersuchungen am Seidenspinner und seinen Verwandten. I. Gametogenese von Bombyx mori L. und Bombyx mandarina $M$. und ihrer Bastarde. $Z$. f. Zellforsch. 7, 519-552 (1928)

39. King, R. C. \& H. AKal: Spermatogenesis in Bombyx mori I. The ring canal system joining sister spermatocytes. J. Morph. 134, 47-55 (1971)

40. King, R. C. \& H. Akal: Spermatogenesis in Bombyx mori II. The ultrastructure of synapsed bivalents. J. Morph. 134, 181-194 (1971)

41. KING, R. C. \& H. AKAl: Observations of meiotic chromosomes with an ultra-high voltage electron microscope. Jeol News 9e, 12-15 (1971)

42. Kitani, Y.: Absence of interference in association with gene conversion in Sordaria fimicola, and presence of interference in association with ordinary recombination. Genetics $89,467-497$ (1978)

43. Koltin, Y. \& J. Stamberg: Genetic control of recombination in Schizophyllum commune. Location of a gene controlling B factor recombination. Genetics 74, 55-62 (1973)

44. Kunde, S. C. \& Yu. F. Bogdanov: Ultrastructural studies of late meiotic prophase nuclei of spermatocytes of Ascaris suum. Chromosoma (Berl.) 70, 375-384 (1979)

45. Liv, L. F., C. C. Liu \& B. M. AlaerTs: Type II DNA topoisomerase: Enzymes that can unknot a topological knotted DNA molecule via a reversible double-strand break. Cell 19, 697-707 (1980)

46. MaEDA, T.: Chiasma studies in the silkworm, Bombyx mori L. Jap. J. Genet. 15, 118-127 (1939)

47. Maguire, M. P.: Homologous chromosome pairing. Phil. Trans. R. Soc. Lond. B. 277, 245258 (1977)

48. Maguire, M. P.: Evidence for separate genetic control of crossing over and chiasma maintenance in maize. Chromosoma (Berl.) 65, 173183 (1978)

49. MaguiRe, M. P.: A possible role for the synaptonemal complex in chiasma maintenance. Exptl. Cell. Res. 112, 297-308 (1978)

50. Mather, K.: Meiosis in Lilium. Cytologia 6, 354-380 (1935)

51. MCCLintock, B.: The stability of broken ends of chromosomes of Zea mays. Genetics 26, 243282 (1941)

52. Moens, P. B.: Multiple core complexes in grasshopper spermatocytes and spermatids. J. Cell Biol. 40, 542-551 (1969)
53. Moens, P. B.: Ultrastructural studies of chiasma distribution. Ann. Rev. Genet. 12, 433-450 (1978)

54. MoEns, P. B.: Lateral element cross connections of the synaptonemal complex and their relationship to crossing over and chiasmata in rat spermatocytes. Canad. J. Genet. Cytol. 20, 567579 (1978)

55. Moens, P. B. \& K. Church: The distribution of synaptonemal complex material in metaphase I bivalents of Locusta and Chloealtis (Orthoptera: Acrididae). Chromosoma (Berl.) 73, 247-254 (1979)

56. Mortimer, R. K. \& S. Fogel: Genetic interference and gene conversion. In: Mechanism of Recombination, R. F. Grell, ed., Plenum Press. New York \& London pp. 263-275 (1974)

57. Moses, M. M.: Synaptonemal complex. Ann. Rev. Genet. 2, 363-412 (1968)

58. Murakami, A. \& H. T. Imai: Cytological evidence for holocentric chromosomes of the silkworm, Bombyx mori and B. mandarina (Bombycidae, Lepidoptera). Chromosoma (Berl.) 47, 167-178 (1974)

59. Nebel, B. R. \& E. M. Coulon: The fine structure of the meiotic chromosomes in pigeon spermatocytes. Chromosoma (Berl.) 13, 139 172 (1972)

60. Parvinen, M. \& K. O. Söderström: Chromosome rotation and formation of synapsis. Nature 260, 534-535 (1976)

61. Perkins, D. D.: Crossing over of flanking markers is less than 50-percent among meiotic convertants. Genetics 91, s94 (1979)

62. Polani, P. E., J. A. Crolla, M. J. Seller \& F. MolR: Meiotic crossing over exchange in the female mouse visualized by BudR substitution. Nature 278, 348-349 (1979)

63. Rasmussen, S. W.: The meiotic prophase in Bombyx mori females analyzed by three dimensional reconstructions of synaptonemal complexes. Chromosoma (Berl.) 54, 245-293 (1976)

64. Rasmussen, S. W.: The transformation of the synaptonemal complex into the selimination chromatin « in Bombyx mori oocytes. Chromosoma (Berl.) 60, 205-221 (1977)

65. Rasmussen, S. W.: Chromosome pairing in triploid females of Bombyx mori analyzed by three dimensional reconstructions of synaptonemal complexes. Carlsberg Res. Commun. 42, 163-197 (1977)

66. Rasmussen, S. W. \& P. B. Holm: Human meiosis II. Chromosome pairing and recombination nodules in human spermatocytes. Carlsberg Res. Commun. 43, 275-327 (1978)

67. Rasmussen, S. W. \& P. B. Holm: Human 
meiosis IV. The elimination of synaptonemal complex fragments from metaphase I bivalents of human spermatocytes. Carlsberg Res. Commun. 43, 423-438 (1979)

68. Rasmussen, S. W. \& P. B. Holm: Chromosome pairing in autotetraploid Bombyx females. Mechanism for exclusive bivalent formation. Carlsberg Res. Commun. 44, 101-125 (1979)

69. Rasmussen, S. W. \& P. B. Holm: Mechanics of meiosis. Hereditas 93, 187-216 (1980)

70. RILEY, R. \& C. N. LAw: Genetic variation in chromosome pairing. Adv. Genet. 13, 57-114 (1965)

71. SADO, T.: Spermatogenesis of the silkworm and its bearing on the radiation induced sterility. Jap. J. Genet. 36 suppl., 136-151 (1961)

72. SANG, H. \& H. L. K. Whitehouse: Genetic recombination at the buff spore locus in Sordaria brevicollis. I. Analysis of flanking marker behaviour in crosses between buff mutants and wild type. Molec. gen. Genet. 174, 327-334 (1979)

73. Solari, A. J.: The behaviour of chromosal axes during diplotene in mouse spermatocytes. Chromosoma (Berl.) 31, 217-230 (1970)

74. SolARI, A. J.: Synaptonemal complexes and associated structures in microspread human spermatocytes. Chromosoma (Berl.) 81, 1-23 (1981)

75. Solari, A. J. \& O. Vilar: Multiple complexes in human spermatocytes. Chromosoma (Berl.) 66 , 331-340 (1978)

76. Stern, H. \& Y. Hotta: Biochemical controls of meiosis. Ann. Rev. Genet. 7, 37-66 (1973)

77. Stern, H. \& Y. Hotta: Regulatory mechanisms in meiotic crossing-over. Ann. Rev. Plant Physiol. 29, 415-436 (1978)

78. Tanaka, Y.: Genetics of the silkworm, Bombyx mori. Adv. Genet. 5, 240-313 (1953)
79. Tazima, Y.: The genetics of the silkworm. Logos Press \& Academic Press London, 253 pp (1964)

80. Tazima, Y., H. DoIra \& H. Akal: The domesticated silkmoth, Bombyx mori. In: Handbook of Genetics, R. C. King ed., Plenum Press, New York \& London Vol. 3 pp 63-124 (1975)

81. Tease, C. \& G. H. Jones: Analysis of exchanges of differentially stained meiotic chromosomes of Locusta migratoria after BrdU-substitution and FPG staining. I. Crossover exchanges in monochiasmate bivalents. Chromosoma (Berl.) 69, 163-178 (1978)

82. WestergaARD, M. \& D. von WetTSTEIN: The meiotic cycle in an ascomycete. In: Effect of radiation on meiotic systems. Internat. Atom. Energy Agency. Panel Proc. Sci. Sti. Pub. 173, pp 113-121 ( 1968)

83. Westergaard, M. \& D. von Wettstein: Studies on the mechanism of crossing-over IV. The molecular organization of the synaptinemal complex in Neottiella (Cooke) Saccardo (Ascomycetes). Compt. Rend. Trav. Lab. Carlsberg 37, 239-268 (1970)

84. WestergaARd, M. \& D. von Wettstein: The synaptinemal complex. Ann. Rev. Genet. 6, $71-$ $110(1972)$

85. Whitehouse, H. L. K.: Towards an understanding of the mechanisms of heredity. 3rd. ed. Edward Arnold, London 528 pp (1973)

86. ZiCKLER, D.: Development of the synaptonemal complex and the »recombination nodules« during meiotic prophase in the seven bivalents of the fungus Sordaria macrospora Auersw. Chromosoma (Berl.) 61, 289-316 (1977)

87. Zickler, D. \& J. M. Simonet: The use of mutants in the analysis of meiosis. In: International Cell Biology 1980-1981, G. Schweiger ed., Springer Verlag, Berlin, Heidelberg \& New York. pp 168-177 (1981) 